\title{
Precipitation of calcium compounds onto rock surfaces in water with cementitious material
}

\section{AUTHOR(S):}

Nara, Yoshitaka; Kashiwaya, Koki; Nakao, Akimasa; Uchida, Hiroto; Hamada, Yusaku; Shibuya, Kazutoshi

\section{CITATION:}

Nara, Yoshitaka ...[et al]. Precipitation of calcium compounds onto rock surfaces in water with cementitious material. Carbonates and Evaporites 2019, 34(3): 1003-1020

\section{ISSUE DATE:}

2019-09

URL:

http://hdl.handle.net/2433/243872

\section{RIGHT:}

This is a post-peer-review, pre-copyedit version of an article published in 'Carbonates and Evaporites'. The final authenticated version is available online at: https://doi.org/10.1007/s13146-018-0471-9; The full-text file will be made open to the public on 22 September 2019 in accordance with publisher's 'Terms and Conditions for Self-Archiving'.; This is not the published version. Please cite only the published version.; この論文は出版社版でありません。引用の際には 出版社版をご確認ご利用ください。 
Precipitation of calcium compounds onto rock surfaces in water with cementitious material

Yoshitaka Nara $^{1, *}$, Koki Kashiwaya ${ }^{1}$, Akimasa Nakao $^{1}$, Hiroto Uchida ${ }^{2}$, Yusaku

Hamada $^{3}$, Kazutoshi Shibuya ${ }^{4}$

1: Department of Civil and Earth Resources Engineering, Graduate School of Engineering, Kyoto

University, Kyoto Daigaku Katsura, Nishikyo-ku, Kyoto 615-8540, Japan

2: Faculty of Engineering, Kyoto University, Kyoto Daigaku Katsura, Nishikyo-ku, Kyoto 615-8540,

Japan

3: Department of Management of Social Systems and Civil Engineering, Graduate School of

Engineering, Tottori University, 4-101 Koyama-Minami, Tottori 680-8552, Japan.

4: Taiheiyo Consultant Co. Ltd., 2-27-8 Higashinihonbashi, Chuo-ku, Tokyo, 103-0004, Japan

*Corresponding author: Yoshitaka Nara

Address: Department of Civil and Earth Resources Engineering, Graduate School of Engineering,

Kyoto University, Kyoto Daigaku Katsura, Nishikyo-ku, Kyoto 615-8540, Japan

E-mail: nara.yoshitaka.2n@kyoto-u.ac.jp, Tel \& Fax: +81 753833210 


\section{Abstract}

In this study, the precipitation of minerals onto rock surfaces was investigated to consider whether sealing of pores and cracks in rock can be accelerated. Cylindrical specimens were prepared and then kept in purified water with powders of high-strength and ultra-low-permeability concrete (HSULPC), which will be used to confine transuranic (TRU) wastes in Japan. Then, the rock specimens were weighed and the surfaces of rock specimens were inspected under a microscope. It was recognized that precipitation occurred on the surface of the rock specimens. It was also shown that precipitation did not occur on rock specimens kept in water without HSULPC. The weight of all specimens stored in HSULPC increased, and the observed weight change was larger for rocks with higher porosities. It is concluded that precipitation of minerals occurs on the rock surface when the rock is kept in water with HSULPC powders. From the results obtained in this study, it is suggested that the sealing of pores and cracks in rock can be accelerated by the precipitation of calcium compounds using HSULPC. It is concluded that HSULPC is useful for underground radioactive waste disposal.

Keywords: Precipitation; Rock; Calcium compound; Scanning electron microscope; Energy dispersion X-ray spectroscopy 


\section{Introduction}

Engineered barriers, such as bentonite, and natural barriers, such as a rock mass, are utilized for the geological disposal of radioactive wastes, reducing the degree of exposure to radionuclides. If a repository of radioactive wastes is located in an area where both the hydraulic gradient and rock permeability are high, the retardation of migration of radionuclides by engineering and natural barriers may not be sufficient.

To retard the migration of radionuclides, several alternative concepts of radioactive waste packaging have been developed (Owada et al. 2005). It is planned that high-strength and ultra-low-permeability concrete (HSULPC) will be used as radioactive waste packaging for the geological disposal of transuranic (TRU) wastes, including ${ }^{14} \mathrm{C}$, in Japan (Kawasaki et al. 2005; Owada et al. 2005). It is intended that the radionuclides will be stored over the long term. Specifically, it is proposed that ${ }^{14} \mathrm{C}$ will be confined for $60 \mathrm{ky}$, corresponding to 10 times the half-life of ${ }^{14} \mathrm{C}$. For this purpose, time-dependent crack propagation and crack sealing in HSULPC have been investigated.

Nara et al. (2010a) investigated the relationship between the crack velocity and stress intensity factor for HSULPC by measuring subcritical crack growth. Atkinson (1984) clarified the effects of the surrounding environment on subcritical crack growth in HSULPC, under various environmental conditions. Fukuda et al. (2012, 2013, 2014) observed the self-sealing of cracks and pores in HSULPC in water using micro-focus 
X-ray computed tomography (CT) and reported that self-sealing occurred by the precipitation of Ca compounds.

A rock mass is expected to perform as a natural barrier, with high confining ability (i.e., a low permeability), for radioactive waste disposal. The permeability of rock is influenced by the distribution and connectivity of pores and cracks in the rock (Gueguen and Dienes 1989; Nara et al. 2011a). If the connectivity of pores and cracks in the rock is poor, then the permeability of the rock is low. The deposition of fine-grained particles is also effective for the sealing of pores and fractures, as reported by Wang et al. (2016). Therefore, a system that accounts for both fine-grained particle deposition and the source of mineral precipitation might ensure the safe disposal of radioactive waste. Usually, the sealing of pores and cracks in rock occurs over geological time scales. If the sealing of pores and cracks in rock can be accelerated by the precipitation of minerals and completed quickly, it will be effective for radioactive waste disposal. It is therefore important to achieve the sealing of pores and cracks in a fast process. The current understanding of mineral precipitation onto rock surfaces over the short term is insufficient for this purpose; therefore, it is necessary to identify whether minerals are able to precipitate on the surfaces of rocks within a short term by laboratory experimentation. Additionally, fine-grained HSULPC particles can enhance the sealing of fractures and pores in rock.

In this study, we investigated the precipitation of minerals and fine-grained particles 
onto rock samples stored in water. Since HSULPC may be used for the geological disposal of TRU wastes, we investigated whether Ca compounds precipitated on the surfaces of rocks kept in water with HSULPC. For comparison, we investigated whether precipitation occurred on the surface of rocks kept in water without HSULPC. The weight of rock specimens before and after the experiment was recorded. An increase in weight would indicate that precipitation had occurred, which can lead to the closure of fractures and pores. 


\section{Samples}

2.1 High-strength and ultra-low-permeability concrete

HSULPC was steam cured at $90^{\circ} \mathrm{C}$ and $99 \%$ relative humidity for 2 days after placing into a mold. Table 1 summarizes the composition of HSULPC. It is recognized that the water cement ratio in HSULPC is lower than that of usual cementitious material. As shown in Table 1, HSULPC includes a large amount of Portland cement, which is more than $60 \%$ comprised of $\mathrm{CaO}$ (Bye et al. 2011). The porosity of HSULPC measured by mercury porosimetry was $5.0 \%$ (Kawasaki et al. 2005). P-wave velocity was $5.0 \mathrm{~km} / \mathrm{s}$, the uniaxial compressive strength was $203 \mathrm{MPa}$, the Brazilian tensile strength was 10.9 MPa, the Young's modulus was 50.9 GPa and the Poisson's ratio was 0.20 (Nara et al. 2010a).

In Figure 1, a scanning electron microscope (SEM) photomicrograph of HSULPC is shown. In this study, pulverized samples (powders) of HSULPC were used when the rock specimens were submerged in water. In Figure 2, an SEM photomicrograph of HSULPC powders used in this study is shown. In this study, the HSULPC powders had a diameter less than $0.2 \mathrm{~mm}$. 


\subsection{Rock samples}

Two igneous rocks, two sandstones, and two carbonate rocks were tested. The igneous rock samples were Oshima granite $(\mathrm{OG})$ and Zimbabwe gabbro $(\mathrm{ZG})$. OG is often used for research and detailed information is available. For the petrological description of OG, please see Sano et al. (1981) and Nara et al. (2010b). ZG used in this study was quarried in the Great Dyke, which is about $50 \mathrm{~km}$ away from Harare in Zimbabwe, and was previously used by Nara et al. (2017a). In Figure 3, the results of X-ray diffraction (XRD) analyses are shown. From this figure, it is recognized that the XRD pattern of ZG shows the distinct peaks of augite, hypersthene, hornblende, plagioclase and quartz. The small peaks of magnetite and illite are also recognized. Sandstone samples used were Berea sandstone (BS) and Shirahama sandstone (SS). These sandstones have been used in our previous studies (Nara et al. 2011b, 2012, 2014). The petrological descriptions of BS and SS were provided by Nara et al. (2011b).

The carbonate rock samples were Macedonian marble (MM) obtained in Skopje and an Italian calcarenite (IC). The MM used in this study was previously used by Nara et al. (2017b). The IC used in this study was previously used by Makhnenko and Labuz (2014). The results of XRD analyses for MM and IC are shown in Figures 4 and 5, respectively. For the XRD pattern of MM, the distinct peak of dolomite and small peaks of calcite, plagioclase and illite are recognized. It has been shown that MM consists 
1

mainly of dolomite, and a few percent of calcite, plagioclase and illite are also present. The XRD pattern of IC suggests that it consists of calcite mainly and a small amount of siderite is also present.

The porosities of the rock samples in this study are summarized in Table 2, which also lists the porosity of HSULPC. 


\section{Experimental method and procedure}

Since this is the first study to investigate precipitation on the surface of rock in water with HSULPC, we used a novel experimental procedure, as follows.

Two cylindrical specimens from each rock sample were prepared. One was placed in purified water with HSULPC powder, and the other was placed in purified water without HSULPC powder. In this study, the surfaces of rock specimens were inspected before and after the experiment, and any change in the weight was noted to identify whether precipitation had occurred.

Once prepared, the cylindrical specimens were kept in an oven at $105^{\circ} \mathrm{C}$ for $24 \mathrm{~h}$ to dry them. The weight of the specimens, in a dry condition, was measured with an electronic analytical scale (AUW220D; SHIMADZU).

Then, the rock specimens were submerged in water-filled plastic containers. Two plastic containers were prepared for each rock sample. One was filled with $150 \mathrm{~g}$ of purified water and $20 \mathrm{~g}$ of HSULPC powder, and another was filled with only purified water. No loads were applied to the specimens during submersion of the rock specimens in water.

The specimens were placed in a chamber, the ESPEC PR-1K, for 2 months. The temperature in the chamber was maintained at $20^{\circ} \mathrm{C}$. Then, the specimens were re-weighed, in the dry condition, to identify any change in their weight. Before weight 
measurements were conducted, the specimens were dried under atmospheric conditions.

If the weight of the rock specimen had increased, it is considered that some precipitation had occurred on the surface of the specimen, which can bring about the closure of fractures and pores existing in rocks.

During the experiment, the water $\mathrm{pH}$ and concentration of $\mathrm{Ca}^{2+}$ cations were monitored using 6367-10D and 6583-10C electrodes (HORIBA), respectively. The accuracy of the $\mathrm{Ca}^{2+}$ concentration measurements was $\pm 10 \mathrm{mg} / \mathrm{L}$. 


\section{Results}

Figure 6 shows photographs of the surfaces of rock specimens before and after submersion in water with powders of HSULPC. The grain boundaries of the specimen kept in water with HSULPC powders became unclear. Few differences were identified on the surfaces of samples submerged without HSULPC powders.

Figure 7 shows photomicrographs of the surfaces of specimens after submersion in water with and without HSULPC powders. Numerous precipitates were observed on all specimens following submergence in water with HSULPC powders, and the pores and cracks were unclear. Notably, needle-shaped precipitates were frequently observed, as well as block-like precipitates; these observations are consistent with those of $\mathrm{Ca}$ compound precipitates on HSULPC by Fukuda et al. (2014).

In all cases, HSULPC powders solidified on the bottom of the containers.

Furthermore, BS and HSULPC specimens stuck to the solidified HSULPC powders. Photographs of the BS and HSULPC specimens stuck to HSULPC powders are shown in Figure $8 \mathrm{a}$ and $\mathrm{b}$, respectively.

We observed the surfaces of rock specimens using a scanning electron microscope / energy dispersive X-ray spectroscopy (SEM/EDX) to clarify the minerals that precipitated on the rock surfaces. SEM photomicrographs and results of elemental mapping on the surfaces of the rock samples kept in water with HSULPC powders are 
1

presented in Figures 9-15. These results suggest that significant $\mathrm{CaCO}_{3}$ precipitation occurred on the surfaces of all samples, because $\mathrm{Ca}, \mathrm{C}$, and $\mathrm{O}$ were detected by the analysis with EDX. In addition, it is considered that $\mathrm{CaMg}\left(\mathrm{CO}_{3}\right)_{2}$ occurred on the surface of OG, ZG, SS, MM and GH, because $\mathrm{Mg}$ was also detected in addition to $\mathrm{Ca}, \mathrm{C}$, and $\mathrm{O}$. 


\section{Discussion}

As mentioned in Section 1, HSULPC will be used as radioactive waste packaging for the geological disposal of TRU wastes (Kawasaki et al. 2005; Owada et al. 2005). If cementitious materials are used underground, then the concentration of $\mathrm{Ca}^{2+}$ in ground water may increase. In such a case, fracture sealing in the rock can be accelerated by the precipitation of $\mathrm{Ca}$ compounds. Therefore, the use of cementitious materials can be advantageous for the geological disposal of radioactive waste. HSULPC powders can produce more $\mathrm{Ca}^{2+}$ in water due to their larger surface area. The preparation of HSULPC powders can facilitate the production of more precipitates of Ca compounds on rock surfaces. In addition, Ishibashi et al. (2016) reported that closure of fractures can result from powder deposition. Therefore, the preparation of powders can also offer advantages in radioactive waste disposal.

Our results demonstrate that $\mathrm{CaCO}_{3}$ and $\mathrm{CaMg}\left(\mathrm{CO}_{3}\right)_{2}$ precipitation occurs on rock surfaces in water with high concentrations of $\mathrm{Ca}$ ions. Precipitation can lead to crack healing, which can decrease rock permeability. Therefore, the results obtained in this study provide fundamental evidence of time-dependent permeability changes in rock.

It is important to consider the source of the precipitate. Figure 16 shows the temporal changes in $\mathrm{Ca}^{2+}$ cation concentration in water. The concentration of $\mathrm{Ca}^{2+}$ increased immediately after the rock samples and HSULPC powders were placed in purified water, 
and then decreased as time elapsed. We presume that the calcium ions were precipitated in solution.

The Ca ion concentration of the purified water used in this study was less than 1 $\mathrm{mg} / \mathrm{L}$. Due to the low concentration of $\mathrm{Ca}$ ions in water, little precipitation was detected on the surfaces of rock samples kept in purified water without HSULPC. To achieve precipitation of $\mathrm{Ca}$ compounds and sealing of fractures in rock, a high concentration of $\mathrm{Ca}^{2+}$ in water is essential. The results shown in Figure 16 suggest that a source of $\mathrm{Ca}^{2+}$ is essential to accelerate the precipitation of $\mathrm{Ca}$ compounds, and to ensure fracture sealing in rock. In addition, our results suggest that HSULPC is a suitable source to increase $\mathrm{Ca}^{2+}$ concentration in water. We therefore recommend the use of HSULPC to effectively ensure the safe geological disposal of radioactive wastes.

Carbonate ions $\left(\mathrm{CO}_{3}{ }^{2-}\right)$ or hydrogen carbonate ions $\left(\mathrm{HCO}_{3}^{-}\right)$are also necessary for the precipitation of $\mathrm{CaCO}_{3}$ or $\mathrm{CaMg}\left(\mathrm{CO}_{3}\right)_{2}$ (Gebrehiwet et al. 2014; Kemache et al. 2016). Usually, $\mathrm{CO}_{3}{ }^{2-}$ or $\mathrm{HCO}_{3}{ }^{-}$ions are present in water. As such, these ions likely contributed to the precipitation of $\mathrm{Ca}$ compounds. In the case of $\mathrm{CaCO}_{3}$ precipitation, the reaction between $\mathrm{Ca}^{2+}$ from $\mathrm{HSULPC}$ and $\mathrm{CO}_{3}{ }^{2-}$ in water may be a possible mechanism. However, the amount of $\mathrm{CO}_{3}{ }^{2-}$ in water may not have been sufficient for the precipitation of calcium compounds, because purified water was used in this study. Therefore, the source of $\mathrm{CO}_{3}{ }^{2-}$ can be $\mathrm{CO}_{2}$ present in the atmosphere, because the specimens used in this study were dried under atmospheric conditions. When the 
specimens were dried, the temperature of the surrounding air was $293 \mathrm{~K}$. The concentration of $\mathrm{CO}_{2}$ in air was around $400 \mathrm{ppm}$, as measured by a $\mathrm{CO}_{2}$ concentration meter. $\mathrm{CO}_{2}$ in the surrounding air is presumed to have dissolved in water, thereby generating $\mathrm{CO}_{3}{ }^{2-}$. The precipitation of $\mathrm{CaMg}\left(\mathrm{CO}_{3}\right)_{2}$ is thought to occur during the chemical reaction between $\mathrm{Ca}^{2+}$ from $\mathrm{HSULPC}, \mathrm{CO}_{3}{ }^{2-}$ from water, and $\mathrm{Mg}^{2+}$ provided by minerals in the rock samples and HSULPC. Since precipitation of carbonate minerals $\left(\mathrm{CaCO}_{3}\right.$ and $\left.\mathrm{CaMg}\left(\mathrm{CO}_{3}\right)_{2}\right)$ occurred on the surfaces of the specimens, it is likely that the concentration of $\mathrm{CO}_{3}{ }^{2-}$ in the water at the surface of the specimens reached the solubility equilibrium when the specimens were dried (Compton and Dary 1987; Donnet et al. 2009). The dissolution of $\mathrm{CO}_{2}$ from the surrounding air may also have contributed to solubility equilibrium.

The temporal changes in the water $\mathrm{pH}$ are shown in Figure 17. Figure 17a and $\mathrm{b}$ show the changes in water $\mathrm{pH}$ with and without HSULPC powders, respectively. The $\mathrm{pH}$ remained almost constant, except at the beginning of the experiment. All rock samples were kept in plastic containers filled with purified water, without opening the lids; for this reason, the change in $\mathrm{pH}$ was small.

A summary of the dry weight data for the rock specimens before and after being kept in water is provided in Table 3. As these data show, the weight of all specimens kept with HSULPC increased, and that of all specimens kept without HSULPC slightly decreased. Since the concentration of $\mathrm{Ca}^{2+}$ in the water was high when the rock was first 
submerged, and then decreased gradually, it is likely that $\mathrm{CaCO}_{3}$ precipitation occurred on the rock surface.

In addition, it is necessary to consider the weight increase due to filling by the powders. It is important to enhance the sealing of fractures and pores in rock to decrease its permeability or prevent an increase of permeability. Therefore, precipitation should be promoted. Ishibashi et al. (2016) reported that fine-grained mineral particles filled the fractures in a rock mass, leading to a decrease in permeability. According to Wang et al. (2016), fillings in rock fractures significantly decrease the permeability of fractured rock. Pérez-Flores et al. (2017) showed that the permeability of fractured rock was decreased by filling even though the fracture was unmated. Nara et al. (2018) reported that the permeability of macro-fractured granite decreased significantly when the macro-fracture was filled naturally by fine-grained minerals. Therefore, it is desirable to produce filling powders to decrease the permeability of fractured rock.

The relationships between the porosity of rocks and changes in the dry weight of specimens are presented in Figure 18. Especially, the changes in the weight of rock samples kept in water with and without HSULPC powders are shown in Figure 18a and $\mathrm{b}$, respectively. Figure 18 a shows that the change in weight of the rock samples was larger when the porosity was high. This result suggests that the weight increase becomes more significant as the amount of precipitation onto the rocks with high porosity increases. Conversely, all specimens kept in water without HSULPC lost mass, which 
indicates that some minerals contained in the rock specimen dissolved in water. The weight reductions observed in these specimens were larger in high-porosity rock samples. Thus, larger rock surface areas increased the amount of dissolution of some minerals.

The main purpose of this study was to investigate whether the precipitation of minerals occurred or not on rock samples in water. Rock samples kept in water with HSULPC increased in weight and the sealing of pores and cracks occurred through the precipitation of $\mathrm{Ca}$ compound and filling by fine-grained mineral particles. If pores and cracks in rock are sealed by the precipitation of Ca compound and filling by fine-grained particles, then the permeability can be decreased. It is therefore desirable to enhance the sealing of pores and cracks by both mineral precipitation from chemical reaction and filling by fine-grained mineral particles. Low permeability (high confining ability) of rock materials is desirable for the underground disposal of radioactive waste. In addition, closure of cracks in rock through precipitation can reduce the crack propagation rate of subcritical crack growth and bring about a subcritical crack growth limit (Atkinson 1984). It is concluded that the precipitation phenomena observed in this study provides important information about the long-term behavior of rocks, which could facilitate radioactive waste disposal projects. 


\section{Conclusion}

In this study, rock specimens were submerged in water, with and without HSULPC, for 2 months under a constant temperature $\left(20^{\circ} \mathrm{C}\right)$ to explore the precipitation of materials onto the rock surfaces. It was recognized that precipitation of some minerals occurred on the surface of rock specimens kept in water with HSULPC powder. Precipitate covered the whole surface. We observed the chemical precipitation of $\mathrm{Ca}$ compounds and the deposition of HSULPC powders, which can promote effective sealing of cracks and pores in rock. Grain boundaries could not be clearly observed on samples kept in water with HSULPC. Conversely, precipitation did not occur on rock specimens kept in water without HSULPC powder. Furthermore, changes in the dry weight were larger for rock specimens with higher porosity. We conclude that the sealing of pores and cracks in rock occurs according to, and is accelerated by, environmental conditions, with high concentrations of calcium ions being provided by HSULPC. Additionally, HSULPC powders are effective for acceleration of the sealing of pores and cracks in rock, and to overcome the cracking and dissolution of rocks. 


\section{References}

Atkinson, B.K., 1984, Subcritical crack growth in geological materials. J Geophys Res, $89,4077-4114$.

Bye, G., Livesey, P., Struble, L. 2011, Portland Cement - Third Edition, ICE Publishing, London.

Compton, R.G. and Daly, P.J., 1987, The dissolution/precipitation kinetics of calcium carbonate: an assessment of various kinetic equations using a rotating disk method. $J$ Colloid Interface Sci, 115, 493-498.

Donnet, M., Bowen, P. and Lemaître, J., 2009. A thermodynamic solution model for calcium carbonate: towards an understanding of multi-equilibria precipitation pathways. J Colloid Interface Sci, 340, 218-224.

Fukuda, D., Nara, Y., Kobayashi, Y., Maruyama, M., Koketsu, M., Hayashi, D., Ogawa, H. and Kaneko, K., 2012, Investigation of self-sealing in high-strength and ultra-low-permeability concrete in water using micro-focus X-ray CT, Cem Concr Res, 42, 1494-1500.

Fukuda, D., Nara, Y., Hayashi, D., Ogawa, H. and Kaneko, K., 2013, Influence of fracture width on sealability in high-strength and ultra-low-permeability concrete in seawater, Materials, 6, 2578-2594.

Fukuda, D., Maruyama, M., Nara, Y., Hayashi, D., Ogawa, H. and Kaneko, K., 2014, Observation of fracture sealing in high-strength and ultra-low-permeability concrete by micro-focus X-ray CT and SEM/EDX, Int J Fract, 188, 159-171.

Gebrehiwet, T., Guo, L., Fox, D., Huang, H., Fujita, Y., Smith, R., Henriksen, J. and Redden, G., 2014, Precipitation of calcium carbonate and calcium phosphate under diffusion controlled mixing, Appl Geochem, 46, 43-56.

Gueguen, Y. and Dienes, J., 1989. Transport properties of rocks from statistics and 
percolation. Math Geol, 21, 1-13.

Ishibashi, M., Yoshida, H., Sasao, E. and Yuguchi, T., 2016. Long term behavior of hydrogeological structures accelerated with faulting: An example from the deep crystalline rock in the Mizunami URL, Central Japan, Eng Geol, 208, 114-127.

Kawasaki, T., Asano, H., Owada, H., Otsuki, A., Yoshida, T., Matsuo, T., Shibuya, K. and Takei, A., 2005, Development of waste package for TRU-disposal (4). Evaluation of confinement performance of TRU waste package made of high strength and ultra low-permeability concrete, Proc. GLOBAL 2005, No. 254.

Kemache, N., Pasquier, L., Mouedhen, I., Cecchi, E., Blais, J. and Mercier, G., 2016, Aqueous mineral carbonation of serpentinite on a pilot scale: the effect of liquid recirculation on $\mathrm{CO}_{2}$ sequestration and carbonate precipitation, Appl Geochem, 67, $21-29$.

Makhnenko, R. Y. and Labuz, J. F., 2014, Calcarenite as a possible host rock for $\mathrm{CO}_{2}$ sequestration, Proc. The 48th US Rock Mechanics / Geomechanics Symposium, Paper No. ARMA14-7559, Minneapolis, Minnesota, USA, June 1-4, 2014.

Nara, Y., Takada, M., Mori, D., Owada, H., Yoneda, T. and Kaneko, K., 2010a, Subcritical crack growth and long-term strength in rock and cementitious material, Int J Fract, 164, 57-71.

Nara, Y., Hiroyoshi, N., Yoneda, T. and Kaneko, K., 2010b, Effects of relative humidity and temperature on subcritical crack growth in igneous rock, Int J Rock Mech Min Sci, 47, 640-646.

Nara, Y., Meredith, P. G., Yoneda, T. and Kaneko, K., 2011a, Influence of macro-fractures and micro-fractures on permeability and elastic wave velocities in basalt at elevated pressure, Tectonophysics, 503, 52-59.

Nara, Y., Morimoto, K., Yoneda, T., Hiroyoshi, N. and Kaneko, K., 2011b, Effects of humidity and temperature on subcritical crack growth in sandstone, Int J Solids Struct, 
$48,1130-1140$.

Nara, Y., Morimoto, K., Hiroyoshi, N., Yoneda, T., Kaneko, K. and Benson, P.M., 2012, Influence of relative humidity on fracture toughness of rock: implications for subcritical crack growth. Int J Solids Struct, 49, 2471-2481.

Nara, Y., Nakabayashi, R., Maruyama, M., Hiroyoshi, N., Yoneda, T. and Kaneko, K., 2014, Influences of electrolyte concentration on subcritical crack growth in sandstone in water, Eng Geol, 179, 41-49.

Nara, Y., Tanaka, M. and Harui, T., 2017a, Evaluating long-term strength of rock under changing environments from air to water, Eng Fract Mech, 178, 201-211.

Nara, Y., Kashiwaya, K., Nishida, Y. and Ii, T., 2017b, Influence of surrounding environment on subcritical crack growth in marble, Tectonophysics, 706-707, $116-128$.

Nara, Y., Kato, M., Niri, R., Kohno, M., Sato, T. Fukuda, D., Sato, T. and Takahashi, M., 2018, Permeability of granite including macro-fracture naturally filled with fine-grained minerals, Pure Appl Geophys, 175, 917-927.

Owada, H., Otsuki, A. and Asano, H., 2005, Development of waste package for TRU-disposal (1). Concepts and performances, Proc. GLOBAL 2005, No.351.

Pérez-Flores, P., Wang, G., Mitchell, T.M., Meredith, P.G., Nara, Y., Sakar, V. and Cembrano, J., 2017, The effect of offset on fracture permeability of rocks from the Andean Southern Volcanic Zone, Chile, J Struct Geol, 104, 142-158.

Sano, O., Ito, I. and Terada, M., 1981, Influence of strain rate on dilatancy and strength of Oshima granite under uniaxial compression, J Geophys Res, 86, 9299-9311.

Wang, G., Mitchell, T.M., Meredith, P.G., Nara, Y. and Wu, Z., 2016, Influence of gouge thickness on permeability of macro-fractured basalt, J Geophys Res - Solid Earth, 121, $8472-8487$. 


\section{Figure captions}

Figure 1 Scanning electron microscope (SEM) photomicrograph of high-strength and ultra-low-permeability concrete (HSULPC).

Figure 2 SEM photomicrograph of HSULPC powders.

Figure 3 X-ray diffraction (XRD) pattern for Zimbabwean gabbro (ZG).

Figure 4 XRD pattern for Macedonian marble (MM) (Nara et al. 2017b).

Figure 5 XRD diffraction pattern for Italian calcarenite (IC).

Figure 6 Photographs of specimen surfaces. (a) Oshima granite (OG) in initial condition; (b) OG following submersion with HSULPC powders; (c) ZG in initial condition; (d) ZG following submersion with HSULPC powders; (e) Berea sandstone (BS) in initial condition; (f) BS following submersion with HSULPC powders; (g) Shirahama sandstone (SS) in initial condition; (h) SS following submersion with HSULPH powders; (i) MM in initial condition; (j) MM following submersion with HSULPH powders; (k) IC in initial condition; (1) IC following submersion with HSULPC powders; (m) HSULPC in initial condition; (n) HSULPC following submersion with HSULPC powders.

Figure 7 SEM photomicrographs of specimen surfaces. (a) OG following submersion in purified water; (b) OG following submersion in water with HSULPC powders; (c) ZG following submersion in purified water; (d) ZG following 
submersion in water with HSULPC powders; (e) BS following submersion in purified water; (f) BS following submersion in water with HSULPC powders; (g) SS following submersion in purified water; (h) SS following submersion in water with HSULPC powders; (i) MM following submersion in purified water; (j) MM following submersion in water with HSULPC powders; (k) IC following submersion in purified water; (1) IC following submersion in water with HSULPC powders; (m) HSULPC following submersion in purified water;

(n) HSULPC following submersion in water with HSULPC powders.

Figure 8 Photographs of specimens of (a) BS and (b) HSULPC stuck to HSULPC powders.

Figure 9 SEM photomicrographs and results of elemental mapping for OG kept in water with HSULPC powders. (a) SEM photomicrograph; (b) elemental mapping for $\mathrm{Ca}$; (c) elemental mapping for $\mathrm{Mg}$; (d) elemental mapping for $\mathrm{C}$; (e) elemental mapping for $\mathrm{O}$.

Figure 10 SEM photomicrographs and results of elemental mapping for ZG kept in water with HSULPC powders. (a) SEM photomicrograph; (b) elemental mapping for $\mathrm{Ca}$; (c) elemental mapping for $\mathrm{Mg}$; (d) elemental mapping for $\mathrm{C}$; (e) elemental mapping for $\mathrm{O}$.

Figure 11 SEM photomicrographs and results of elemental mapping for BS kept in water with HSULPC powders. (a) SEM photomicrograph; (b) elemental mapping 
for $\mathrm{Ca}$; (c) elemental mapping for $\mathrm{C}$; (d) elemental mapping for $\mathrm{O}$.

Figure 12 SEM photomicrographs and results of elemental mapping for SS kept in water with HSULPC powders. (a) SEM photomicrograph; (b) elemental mapping for $\mathrm{Ca}$; (c) elemental mapping for $\mathrm{Mg}$; (d) elemental mapping for C; (e) elemental mapping for $\mathrm{O}$.

Figure 13 SEM photomicrographs and results of elemental mapping for MM kept in water with HSULPC powders. (a) SEM photomicrograph; (b) elemental mapping for $\mathrm{Ca}$; (c) elemental mapping for $\mathrm{Mg}$; (d) elemental mapping for C; (e) elemental mapping for $\mathrm{O}$.

Figure 14 SEM photomicrographs and results of elemental mapping for IC kept in water with HSULPC powders. (a) SEM photomicrograph; (b) elemental mapping for $\mathrm{Ca}$; (c) elemental mapping for $\mathrm{Mg}$; (d) elemental mapping for C; (e) elemental mapping for $\mathrm{O}$.

Figure 15 SEM photomicrographs and results of elemental mapping for HSULPC kept in water with HSULPC powders. (a) SEM photomicrograph; (b) elemental mapping for $\mathrm{Ca}$; (c) elemental mapping for $\mathrm{Mg}$; (d) elemental mapping for $\mathrm{C}$; (e) elemental mapping for $\mathrm{O}$.

Figure 16 Temporal changes in $\mathrm{Ca}^{2+}$ cation concentration in water where samples were kept with HSULPC powders.

Figure 17 Temporal changes in $\mathrm{pH}$ in water (a) with and (b) without HSULPC 
1

2

3

4

5

6

7

8

9

10

11

12

13

14

15

16

17

18

19

20

21

22

23

24

25

26

27

28

29

30

31

32

33

34

35

36

37

38

39

40

41

42

43

44

45

46

47

48

49

50

51

52

53

54

55

56

57

58

59

60

61

62

63

64

65

powders.

Figure 18 Changes in the weights of samples kept in water (a) with and (b) without HSULPC powders. 


\section{Table captions}

Table 1 Composition of high-strength and ultra-low-permeability concrete (HSULPC), after Nara et al. (2010a).

Table 2 Porosity of samples.

Table 3 Summary of dry weight of samples before and after the experiment.

Table 1 Composition of high-strength and ultra-low-permeability concrete (HSULPC), after Nara et al. (2010a).

\begin{tabular}{cc}
\hline & Amount $\left(\mathrm{kg} / \mathrm{m}^{3}\right)$ \\
\hline Low-heat Portland cement & $744-1,014$ \\
Silica fume & $158-496$ \\
Fillers (fly ash, blast furnace slag, etc.) & $225-541$ \\
Aggregates & $631-947$ \\
Water-reducing admixture & 24 \\
Water & 180 \\
\hline
\end{tabular}


1

Table 2 Porosity of samples.

\begin{tabular}{cc}
\hline Samples & Porosity $(\%)$ \\
\hline Oshima granite & 0.37 \\
Zimbabwe gabbro & 0.65 \\
Berea sandstone & 20.4 \\
Shirahama sandstone & 12.9 \\
Macedonian marble & 0.60 \\
Italian calcarenite & 35.4 \\
HSULPC & 5.0 \\
\hline
\end{tabular}


Table 3 Summary of dry weight of samples before and after the experiment.

\begin{tabular}{|c|c|c|c|}
\hline Sample & $\begin{array}{l}\text { Dry weight before } \\
(\mathrm{g})\end{array}$ & $\begin{array}{l}\text { Dry weight after } \\
(\mathrm{g})\end{array}$ & $\begin{array}{c}\text { Weight change } \\
(\mathrm{g})\end{array}$ \\
\hline $\begin{array}{c}\text { Oshima granite } \\
\text { with HSULPC powders }\end{array}$ & 20.361 & 20.366 & 0.005 \\
\hline $\begin{array}{c}\text { Oshima granite } \\
\text { without HSULPC powders }\end{array}$ & 21.105 & 21.101 & -0.004 \\
\hline $\begin{array}{c}\text { Berea sandstone } \\
\text { with HSULPC powders }\end{array}$ & 15.759 & N/A & N/A \\
\hline $\begin{array}{l}\text { Berea sandstone } \\
\text { without HSULPC powders }\end{array}$ & 15.622 & 15.607 & -0.015 \\
\hline $\begin{array}{l}\text { Shirahama sandstone } \\
\text { with HSULPC powders }\end{array}$ & 17.615 & 17.643 & 0.028 \\
\hline $\begin{array}{c}\text { Shirahama sandstone } \\
\text { without HSULPC powders }\end{array}$ & 17.449 & 17.411 & -0.038 \\
\hline $\begin{array}{c}\text { Macedonian marble } \\
\text { with HSULPC powders }\end{array}$ & 21.287 & 21.290 & 0.003 \\
\hline $\begin{array}{c}\text { Macedonian marble } \\
\text { without HSULPC powders }\end{array}$ & 21.762 & 21.750 & -0.012 \\
\hline $\begin{array}{c}\text { Zimbabwe gabbro } \\
\text { with HSULPC powders }\end{array}$ & 24.085 & 24.091 & 0.006 \\
\hline $\begin{array}{l}\text { Zimbabwe gabbro } \\
\text { without HSULPC powders }\end{array}$ & 23.992 & 23.988 & -0.004 \\
\hline $\begin{array}{c}\text { Italian calcarenite } \\
\text { with HSULPC powders }\end{array}$ & 13.149 & 13.170 & 0.021 \\
\hline $\begin{array}{c}\text { Italian calcarenite } \\
\text { without HSULPC powders }\end{array}$ & 13.301 & 13.281 & -0.020 \\
\hline $\begin{array}{c}\text { HSULPC } \\
\text { with HSULPC powders }\end{array}$ & 19.423 & N/A & N/A \\
\hline $\begin{array}{c}\text { HSULPC } \\
\text { without HSULPC powders }\end{array}$ & 18.997 & 18.905 & -0.092 \\
\hline
\end{tabular}


1

2

3

4

5

6

7

8

9

10

11

12

13

14

15

16

17

18

19

20

21

22

23

24

25

26

27

28

29

30

31

32

33

34

35

36

37

38

39

40

41

42

43

44

45

46

47

48

49

50

51

52

53

54

55

56

57

58

59

60

61

62

63

64

65

The English in this document has been checked by at least two professional editors, both native speakers of English. For a certificate, please see:

http://www.textcheck.com/certificate/Djj1wv 


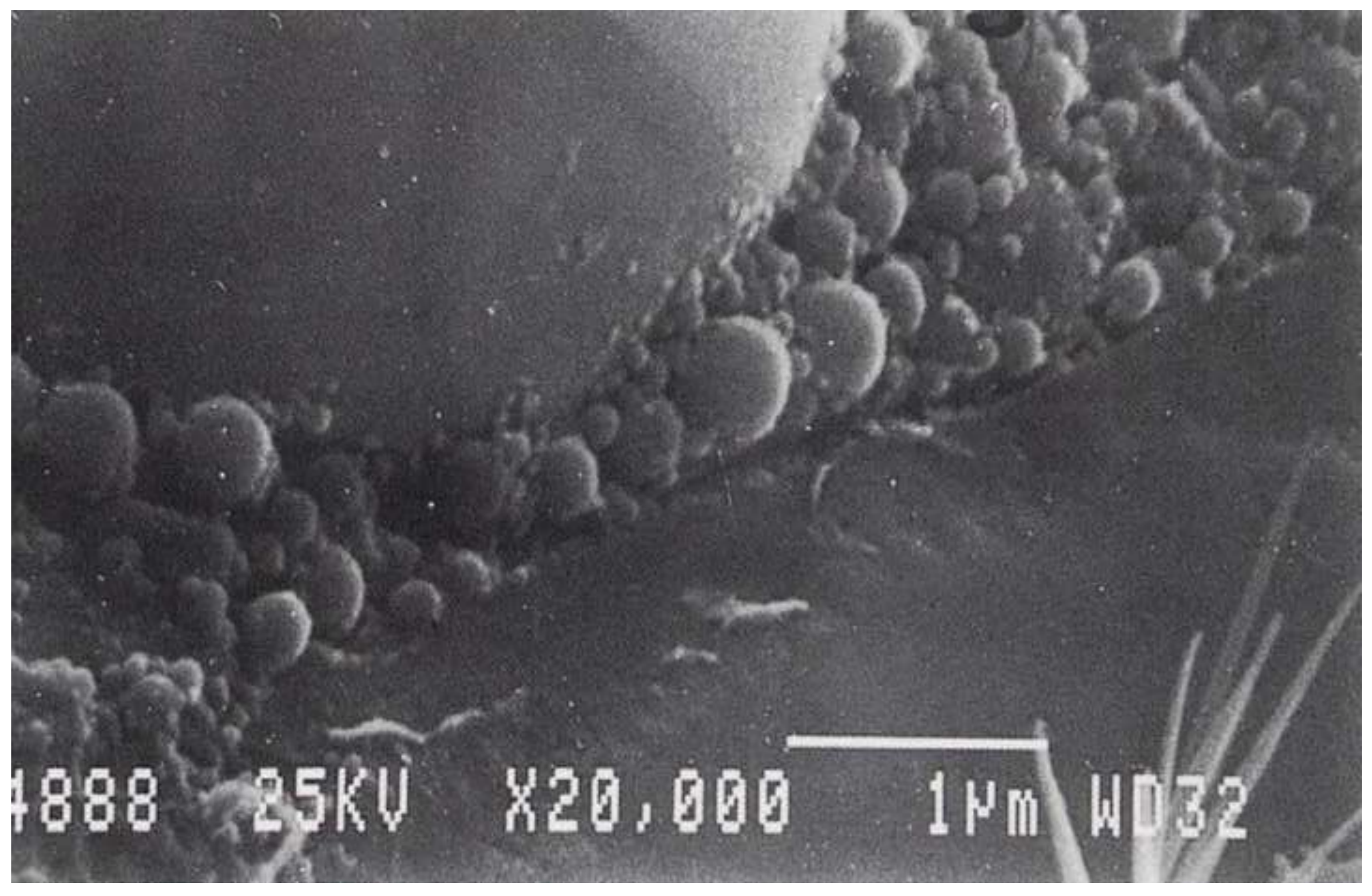




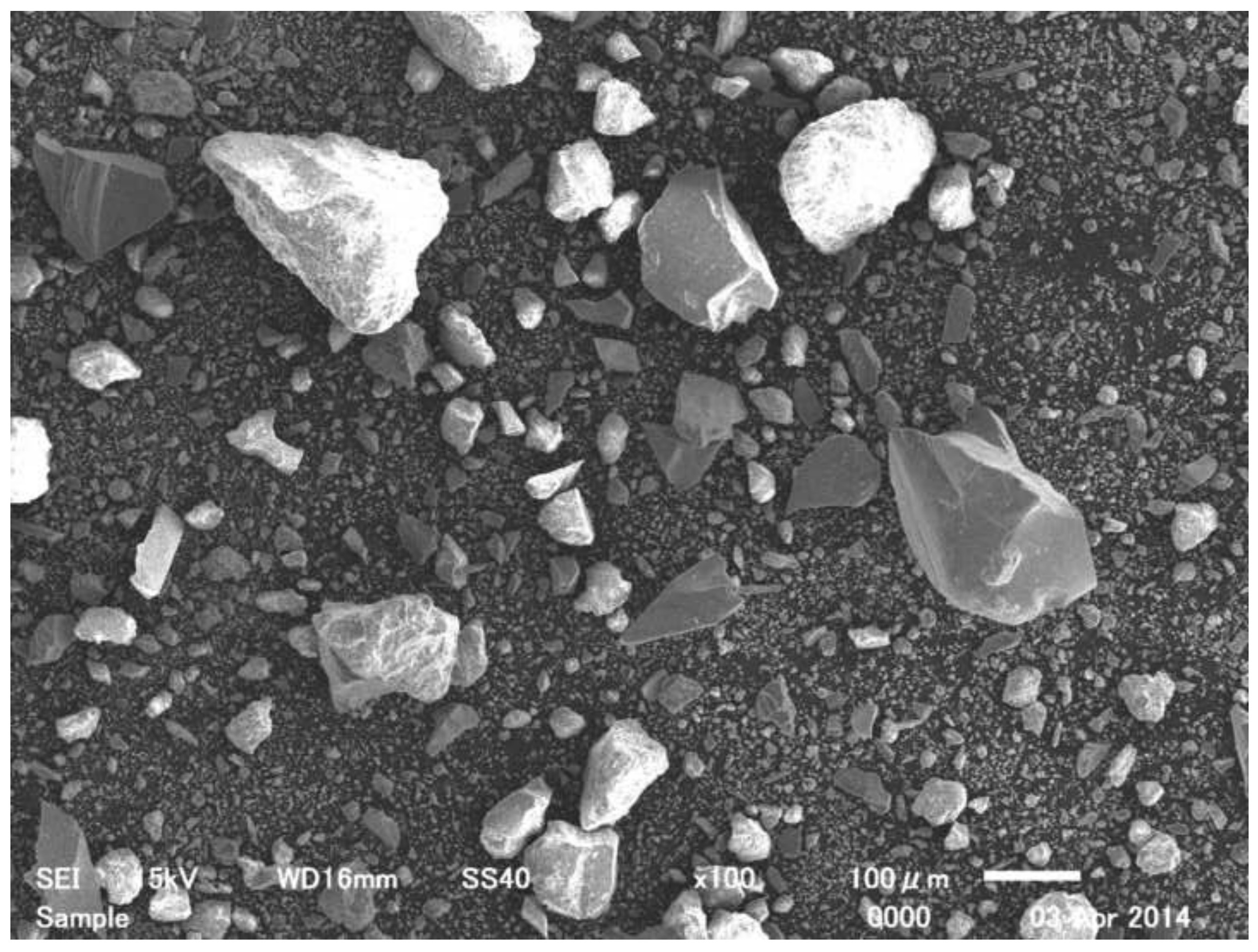




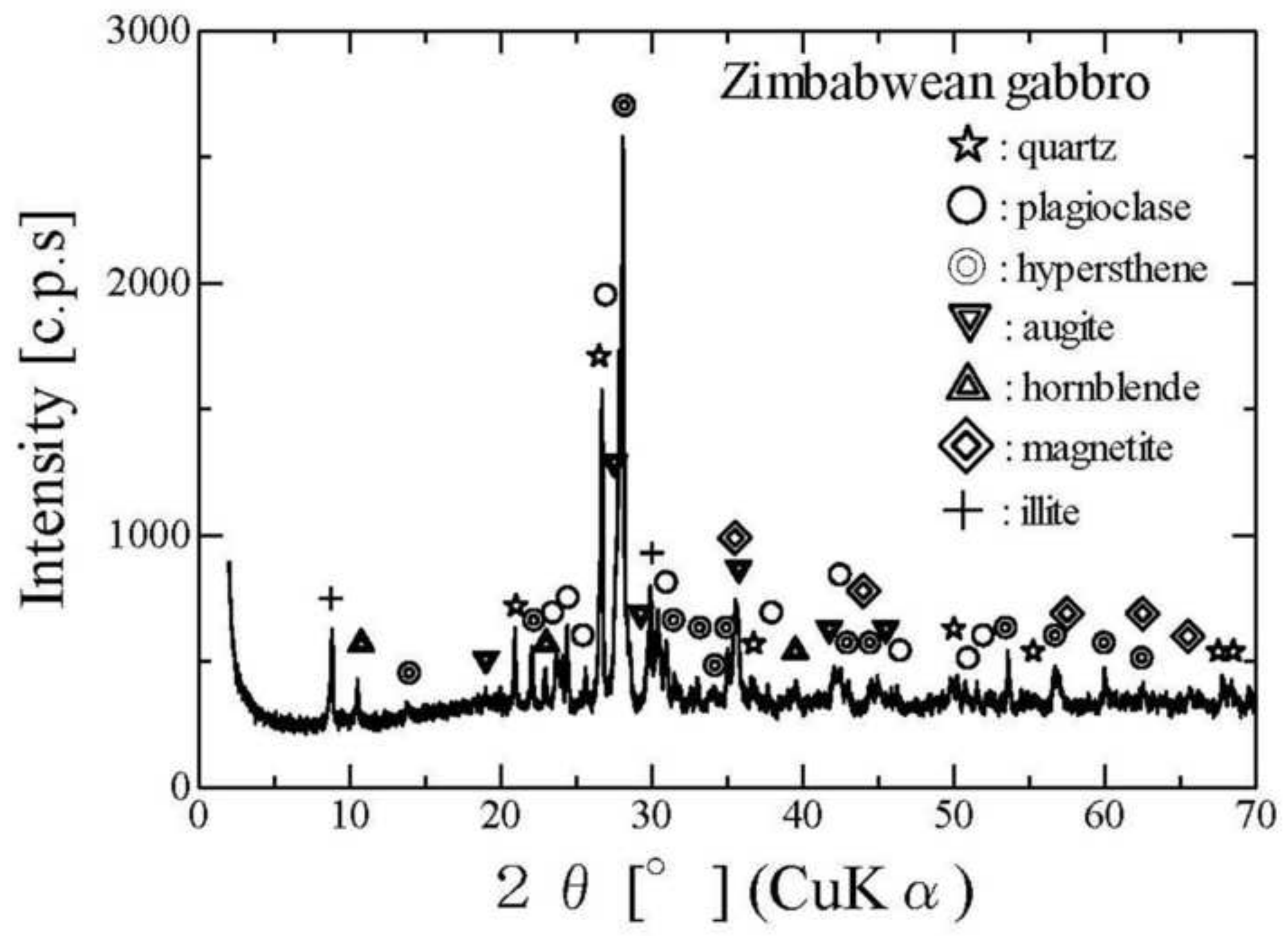




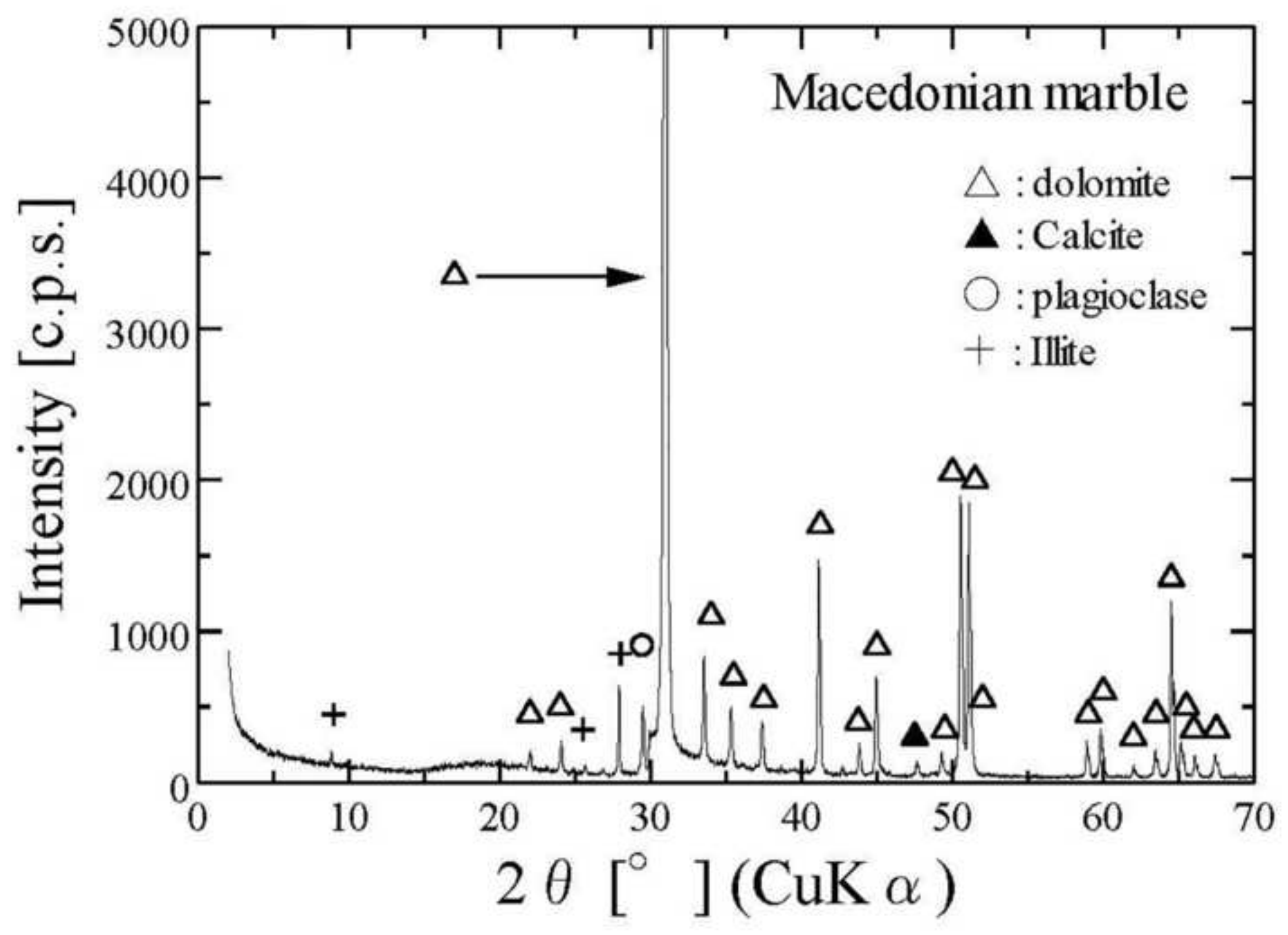




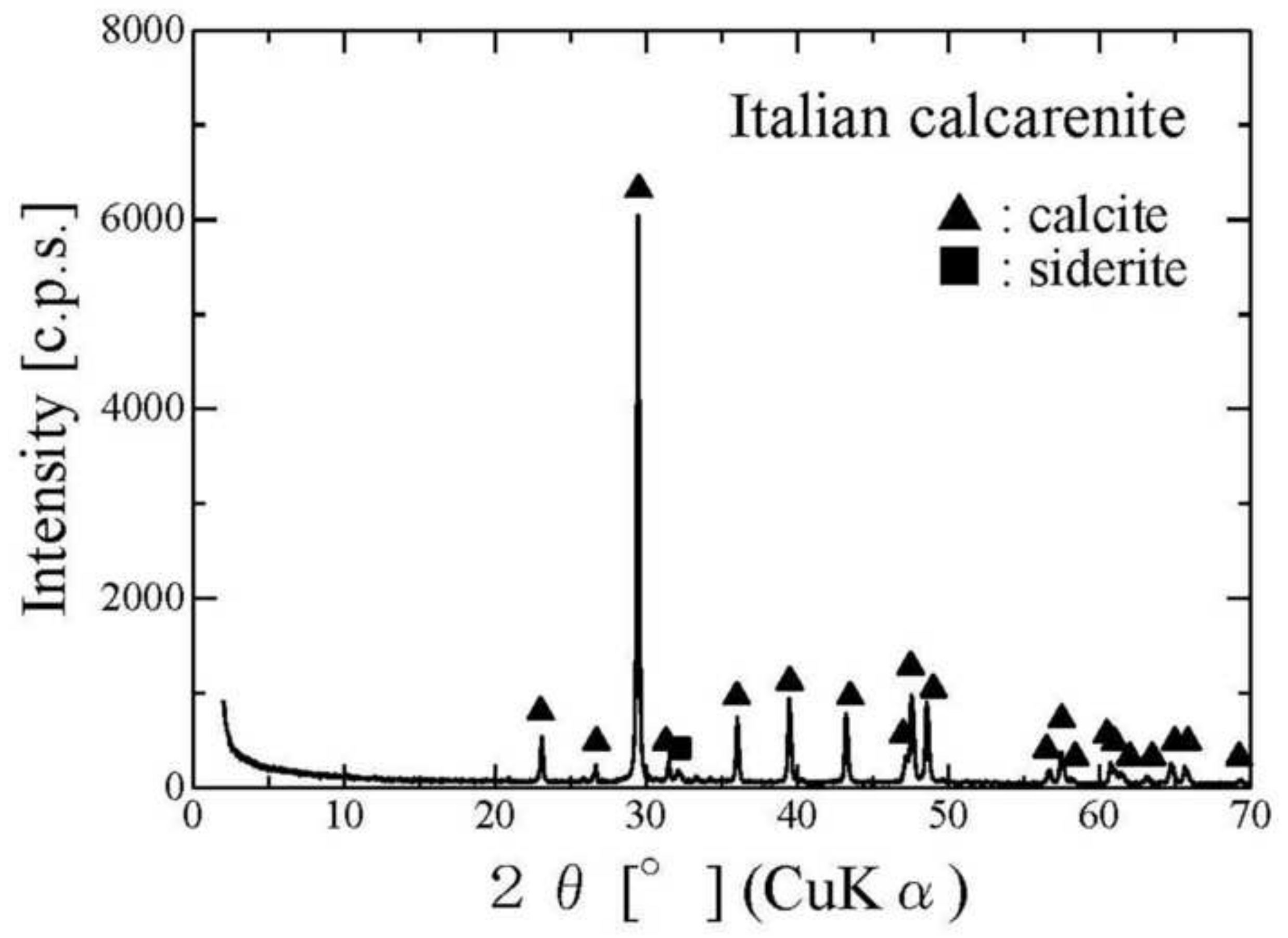


Click here to download Figure Fig_06a(OG1_initial).jpg $\underline{\underline{\underline{ }}}$

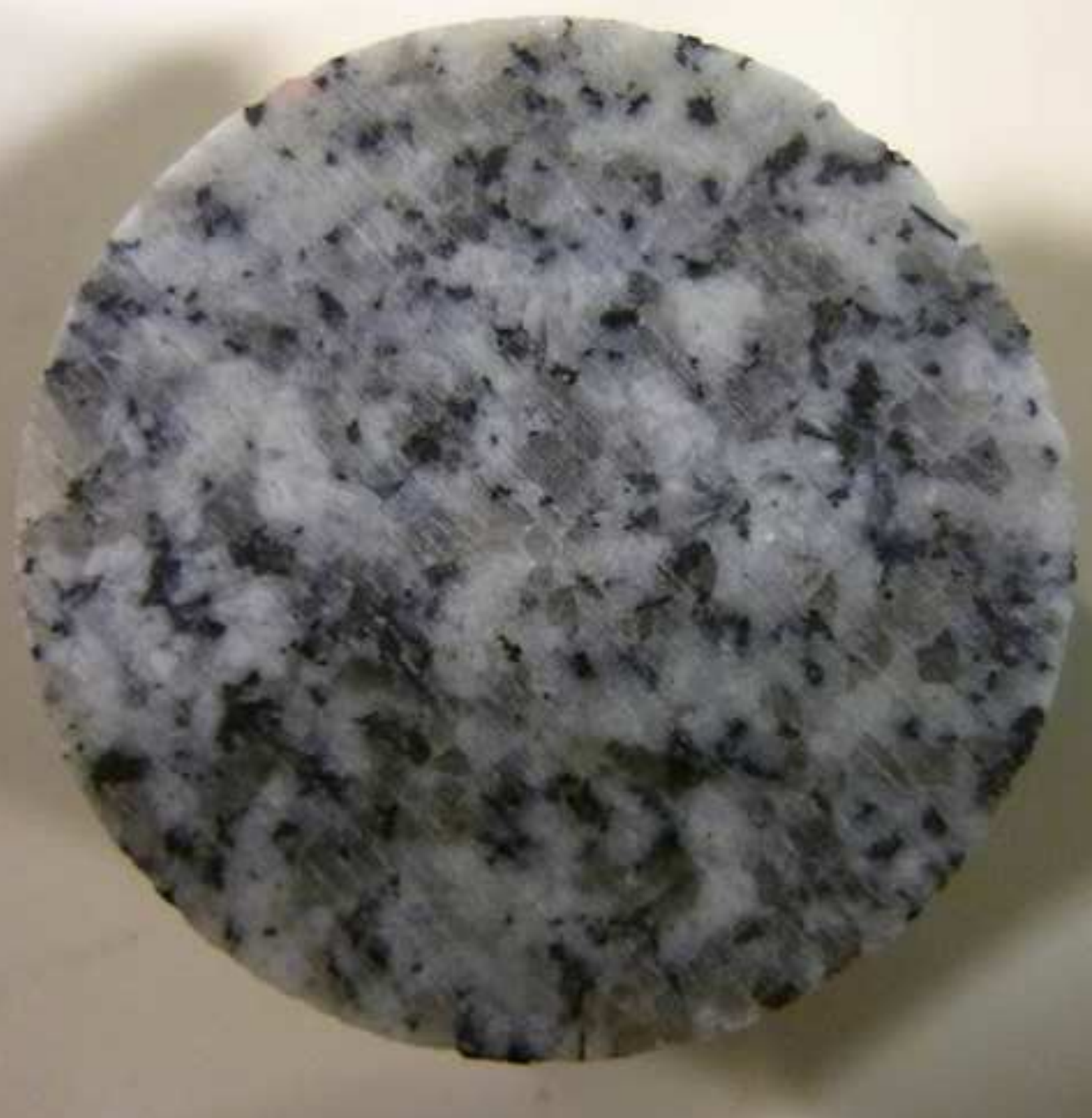




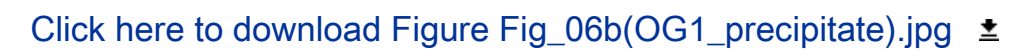

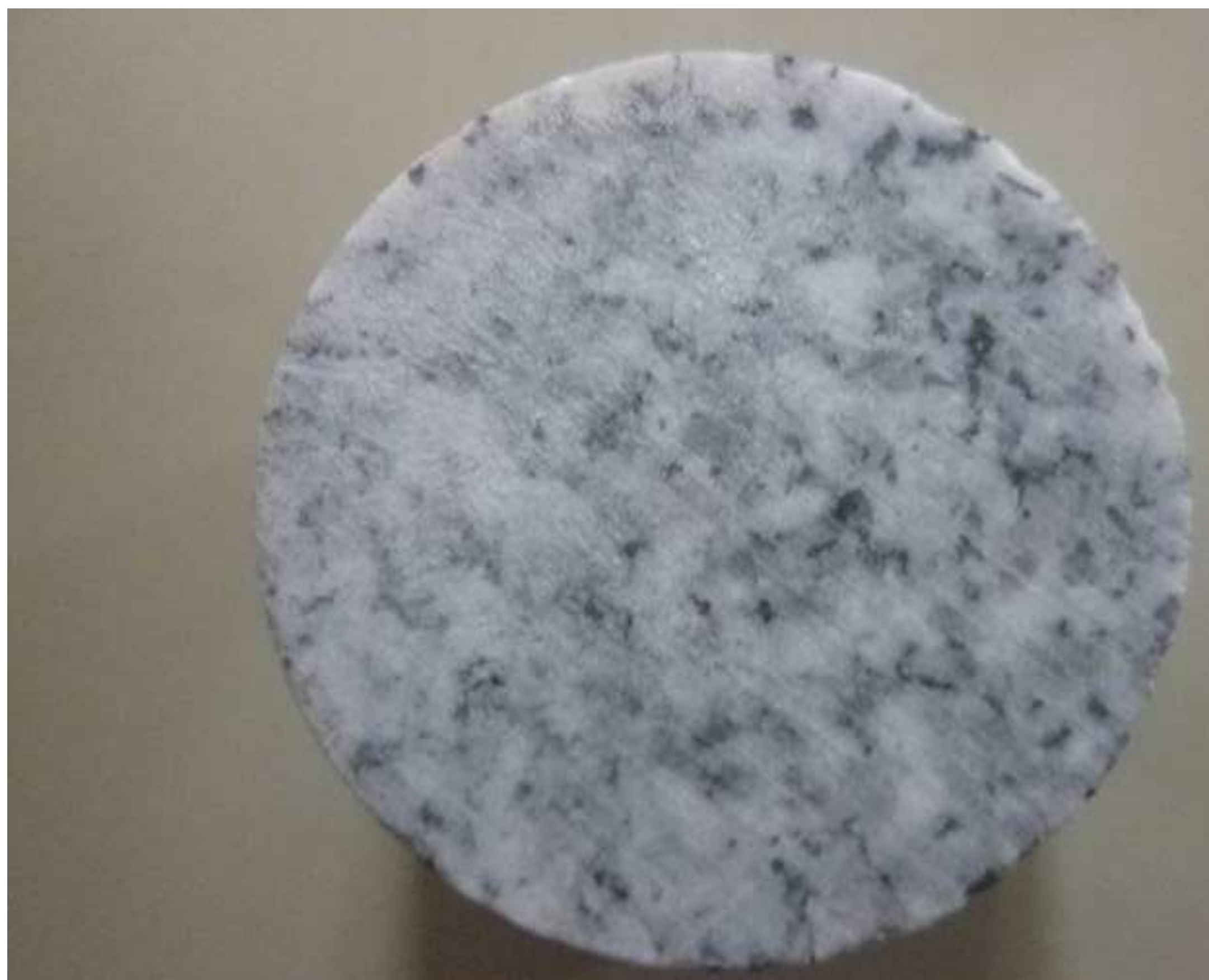

(10)

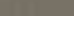

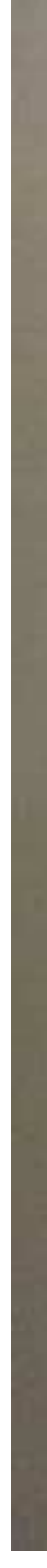


Click here to download Figure Fig_06c(ZG1_initial).jpg $\stackrel{ \pm}{ \pm}$
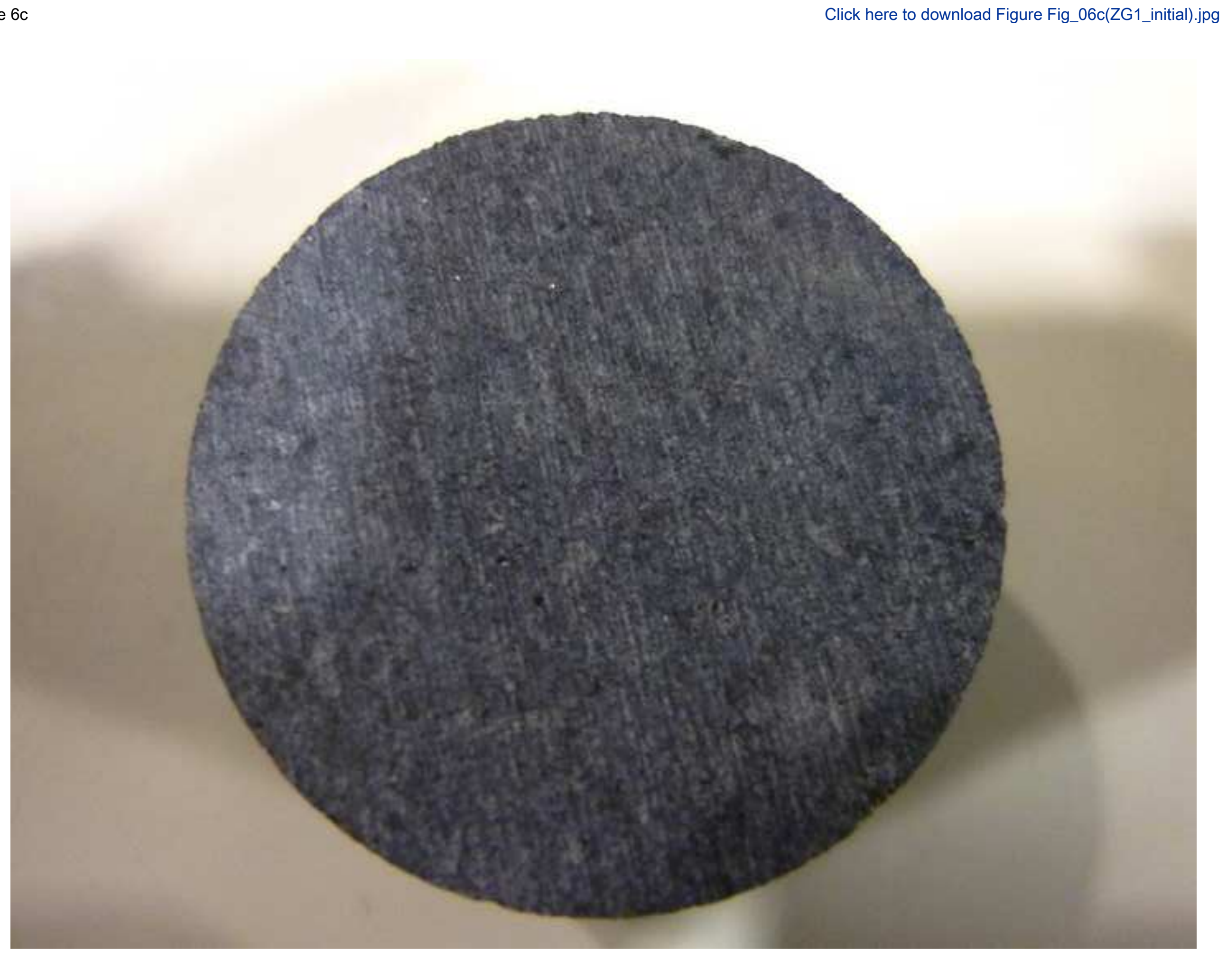

\title{
figure 60

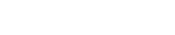

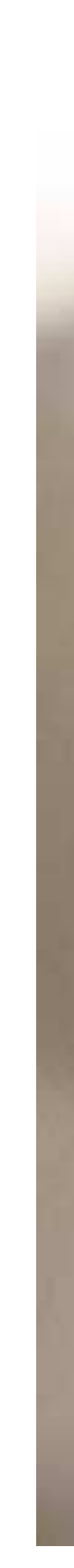

\author{
.
}

.

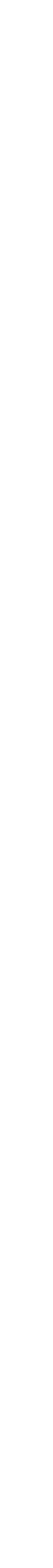



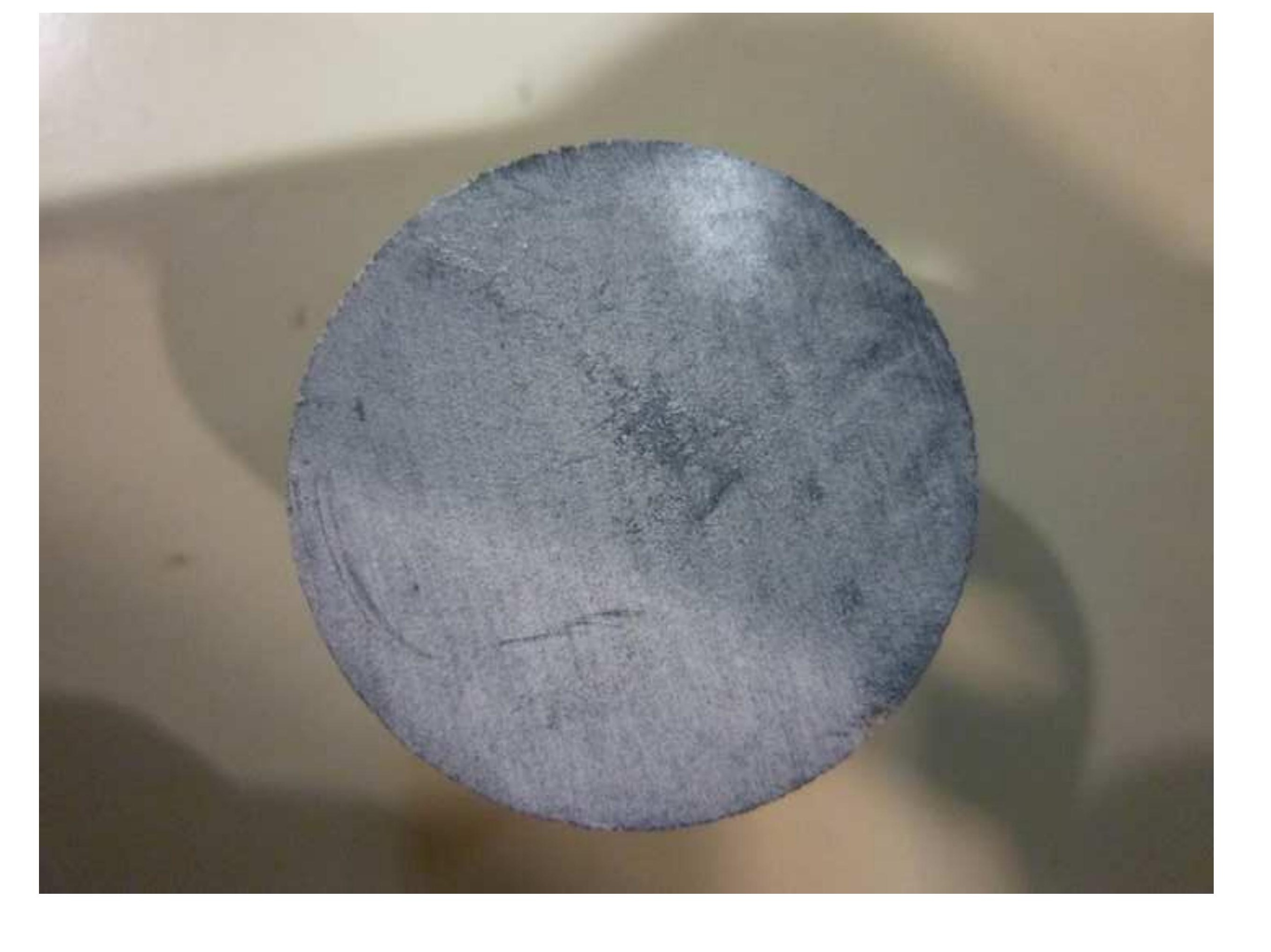
Figure

.
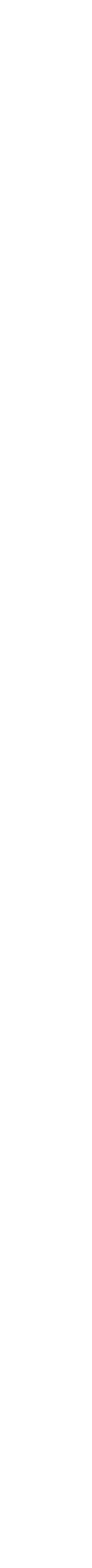


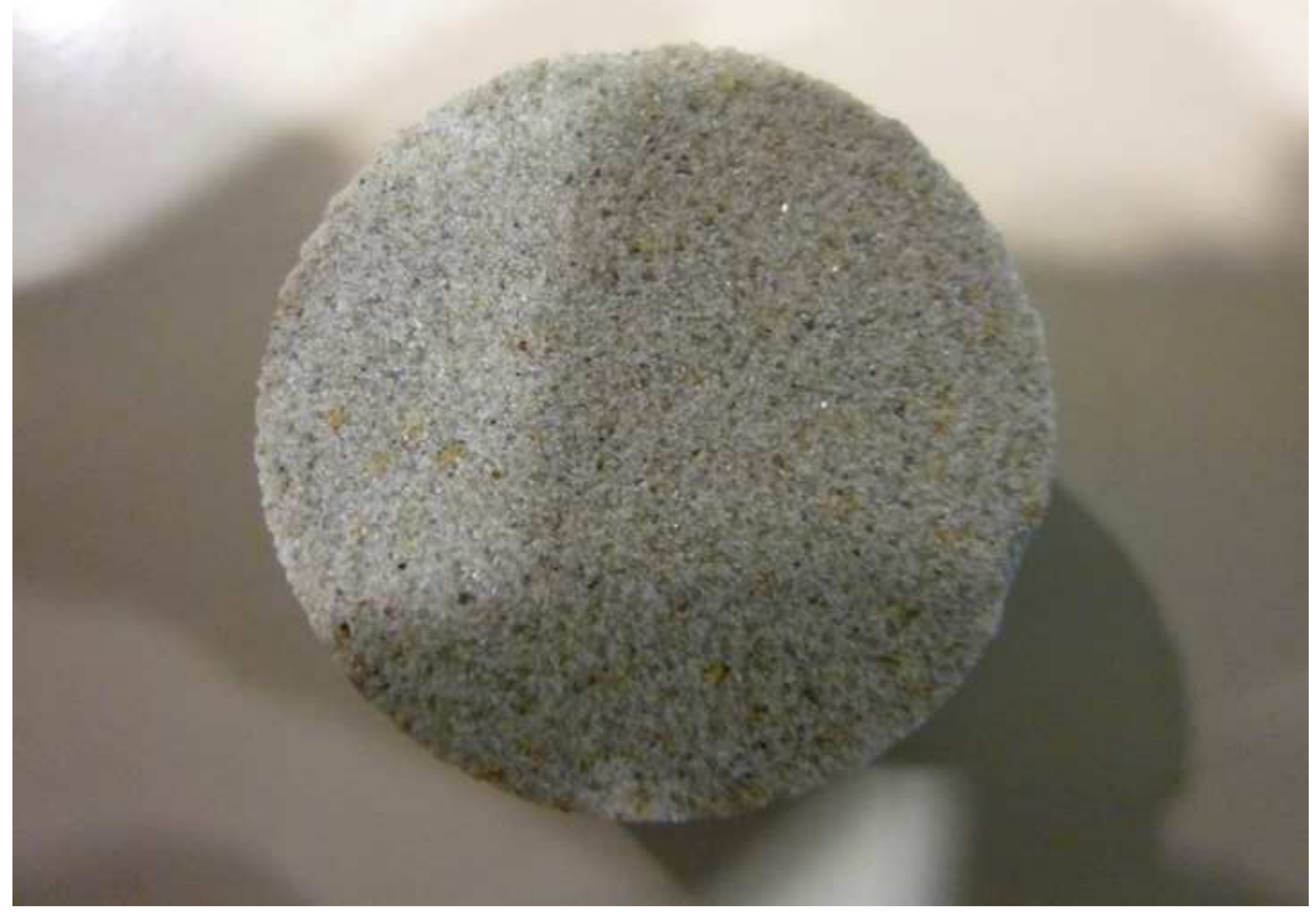




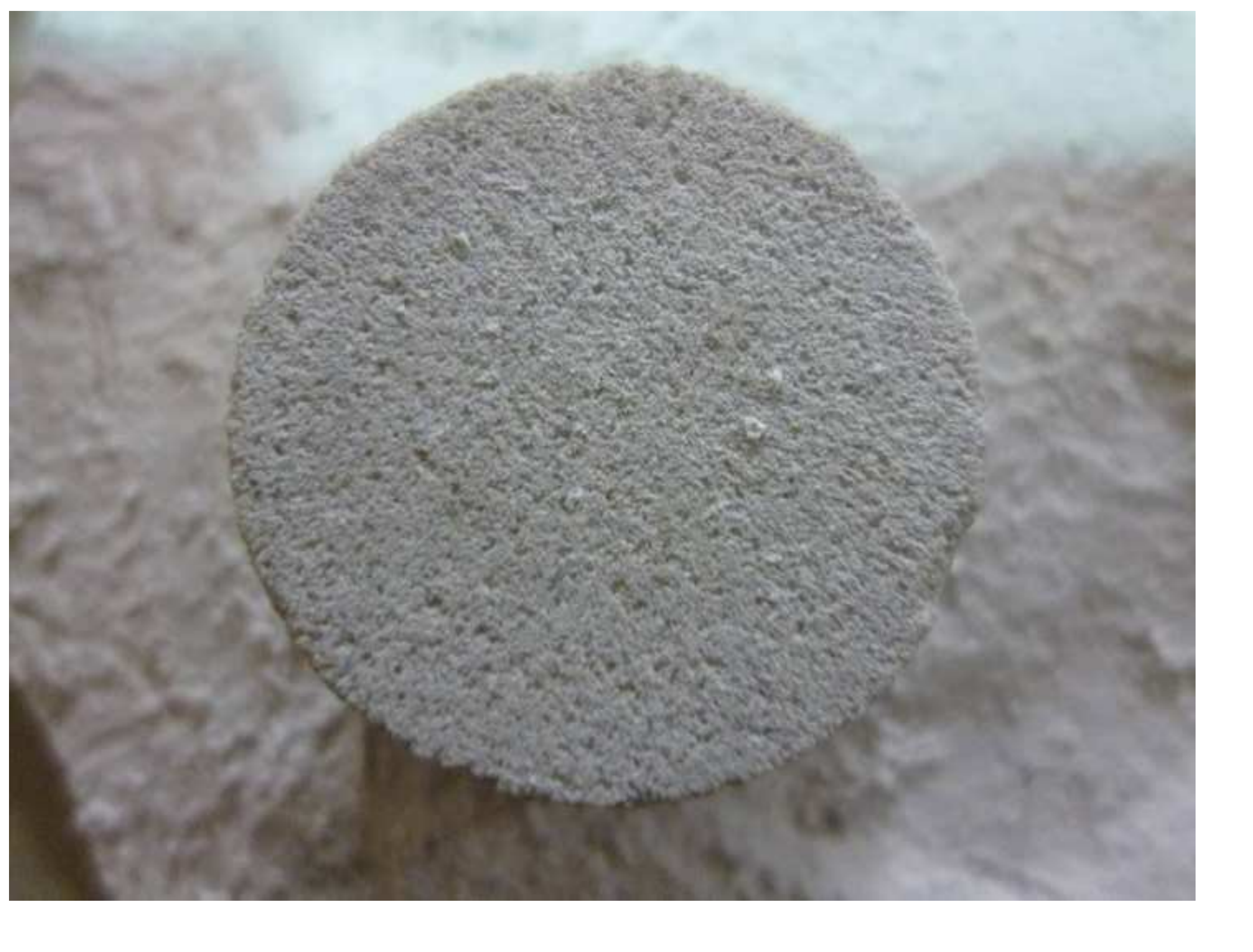
. 
Figure $6 \mathrm{i}$

Click here to download Figure Fig_06i(MM1_initial).jpg $\underline{\underline{\underline{ }}}$
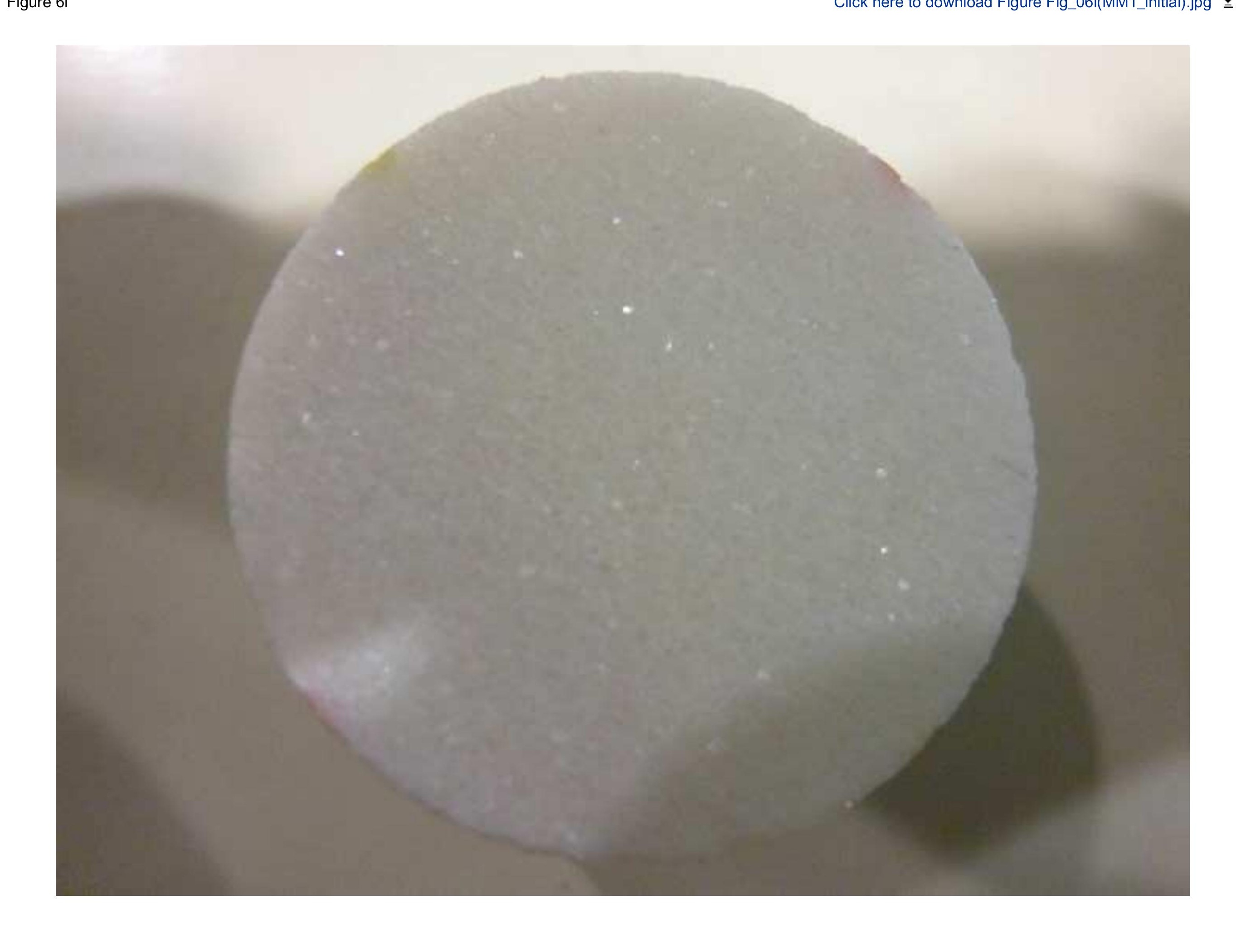


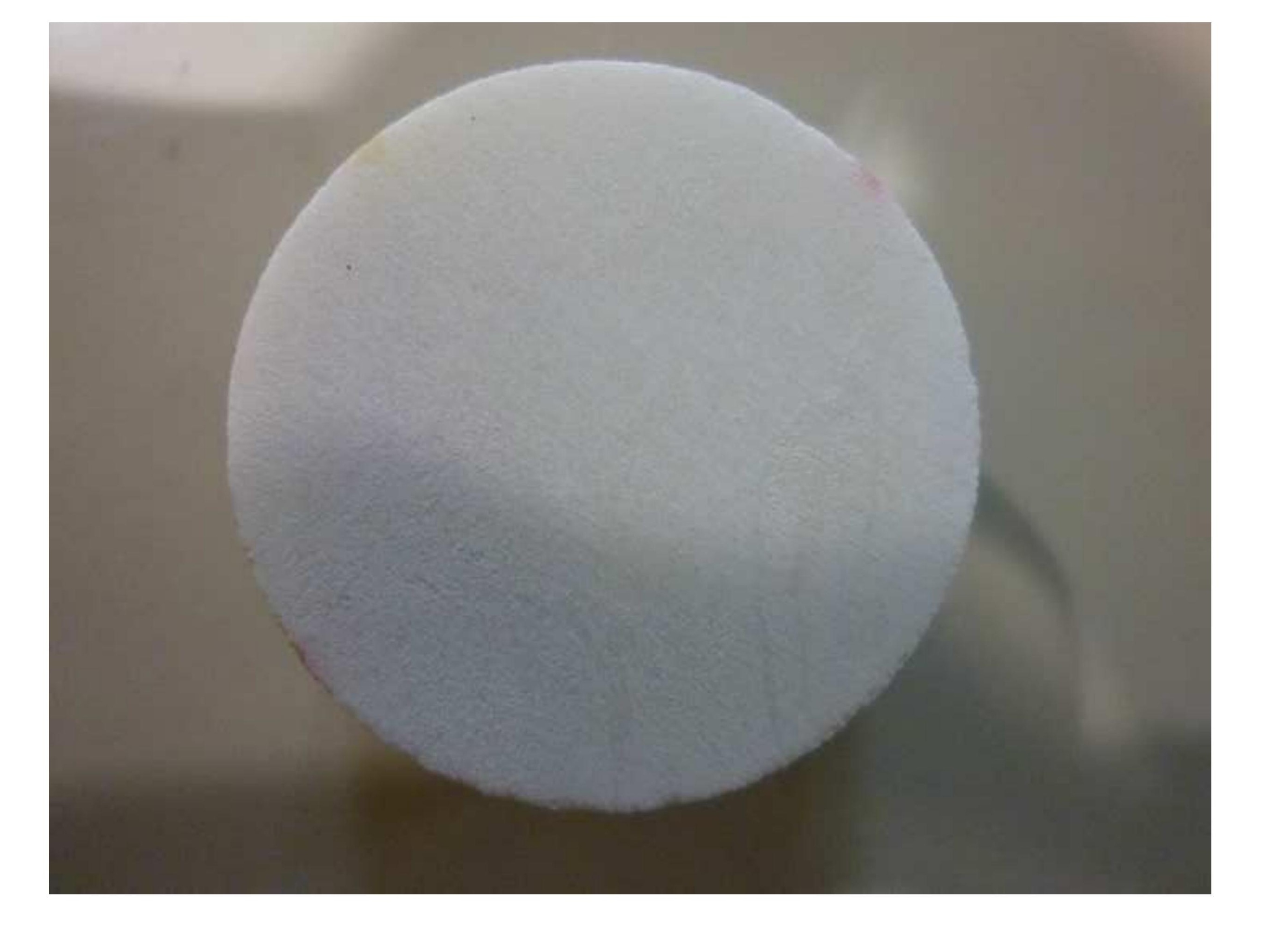
.
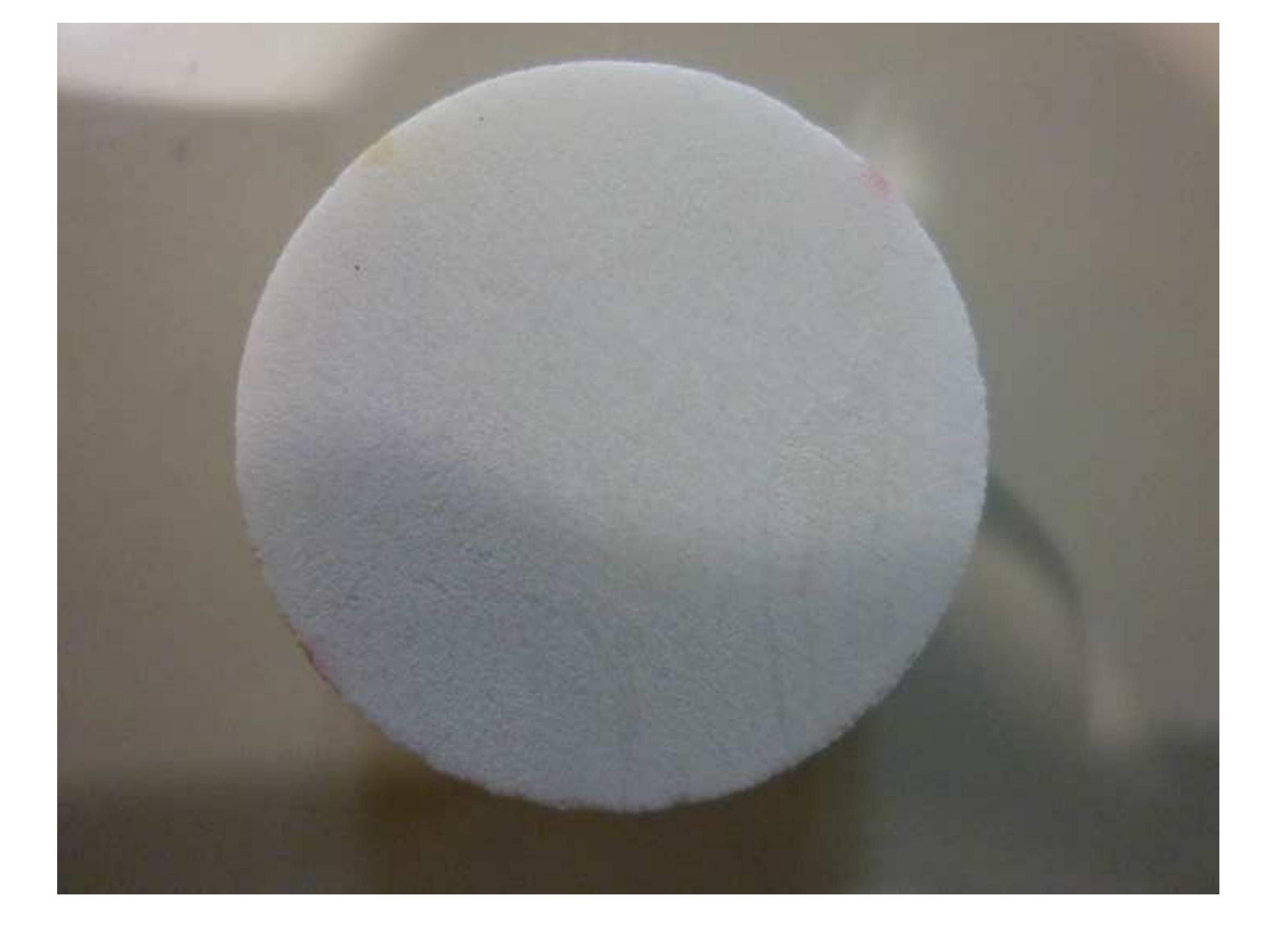
Click here to download Figure Fig_06k(IC1_initial).jpg $\underline{\underline{\underline{ }}}$

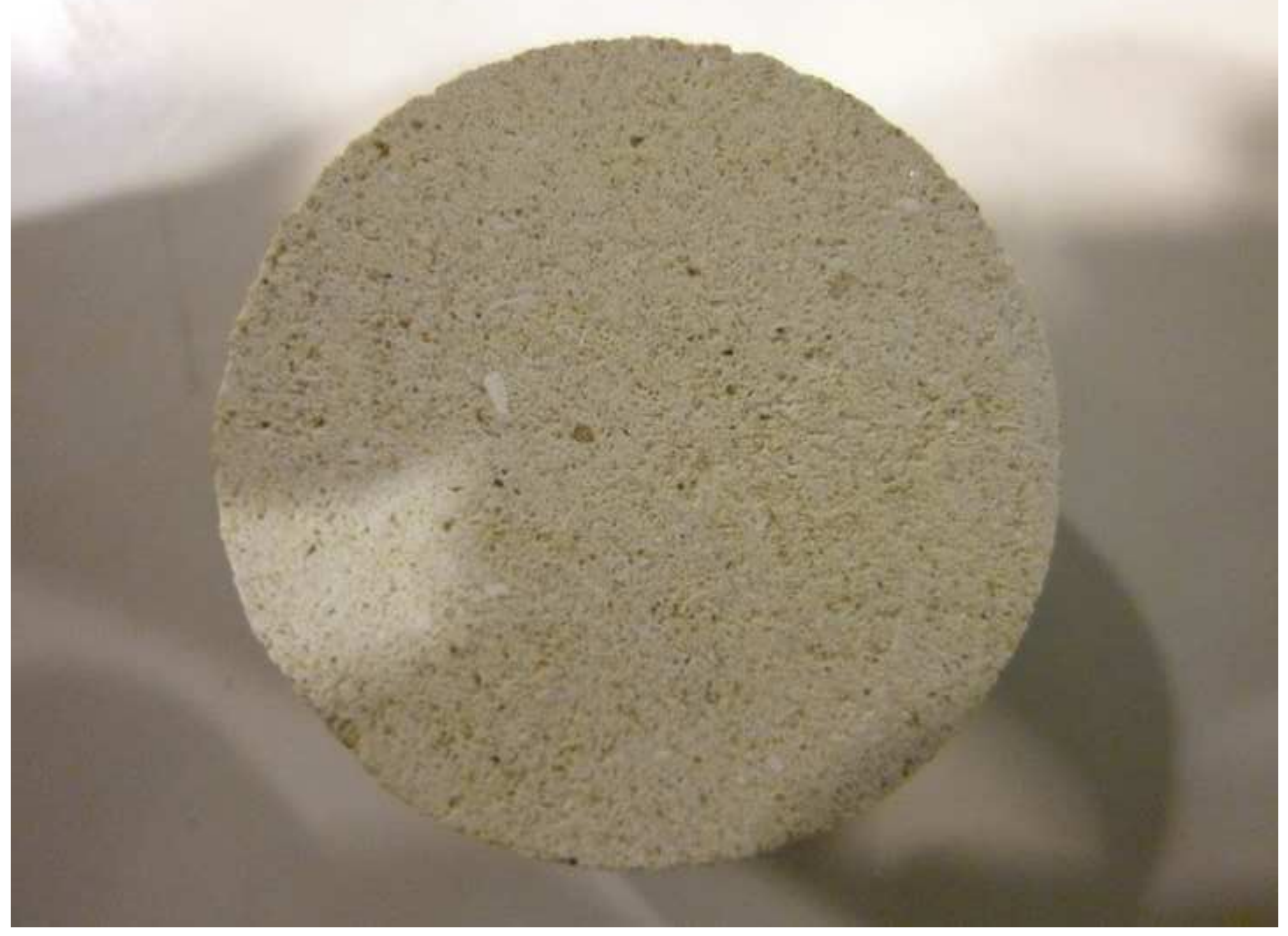




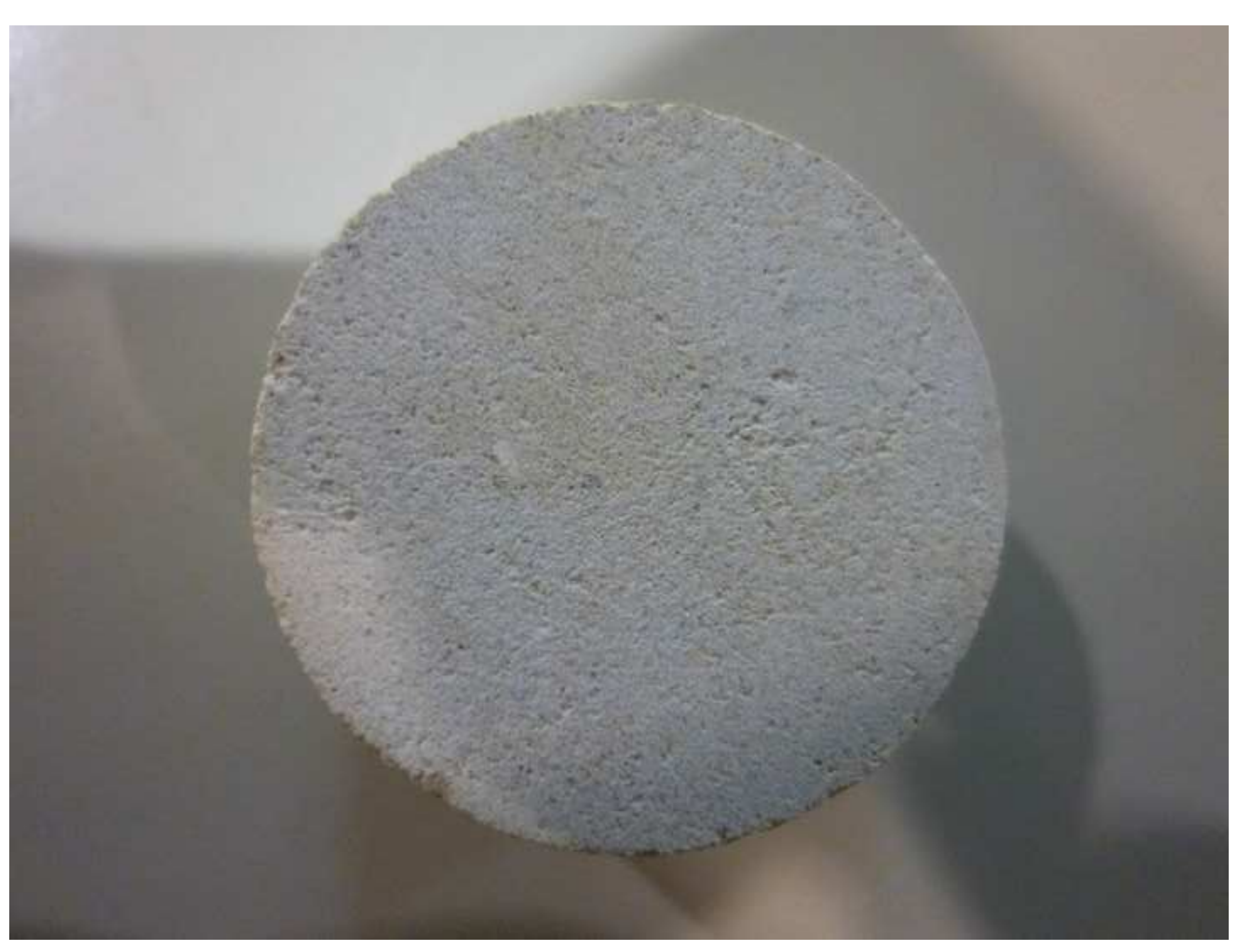




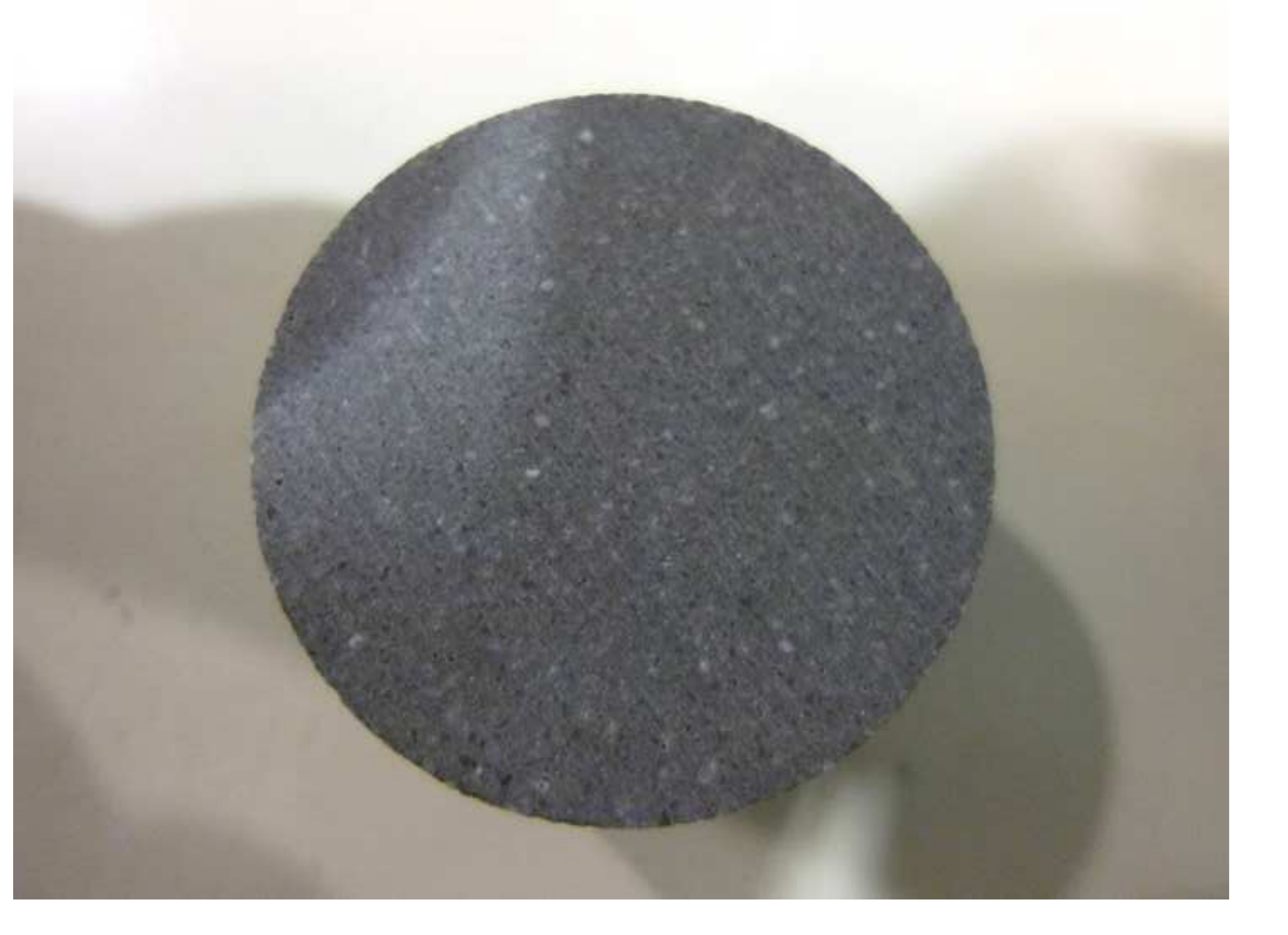

Click here to download Figure Fig_06m(GH1_initial).jpg $\stackrel{\underline{\underline{ }}}{ }$

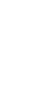
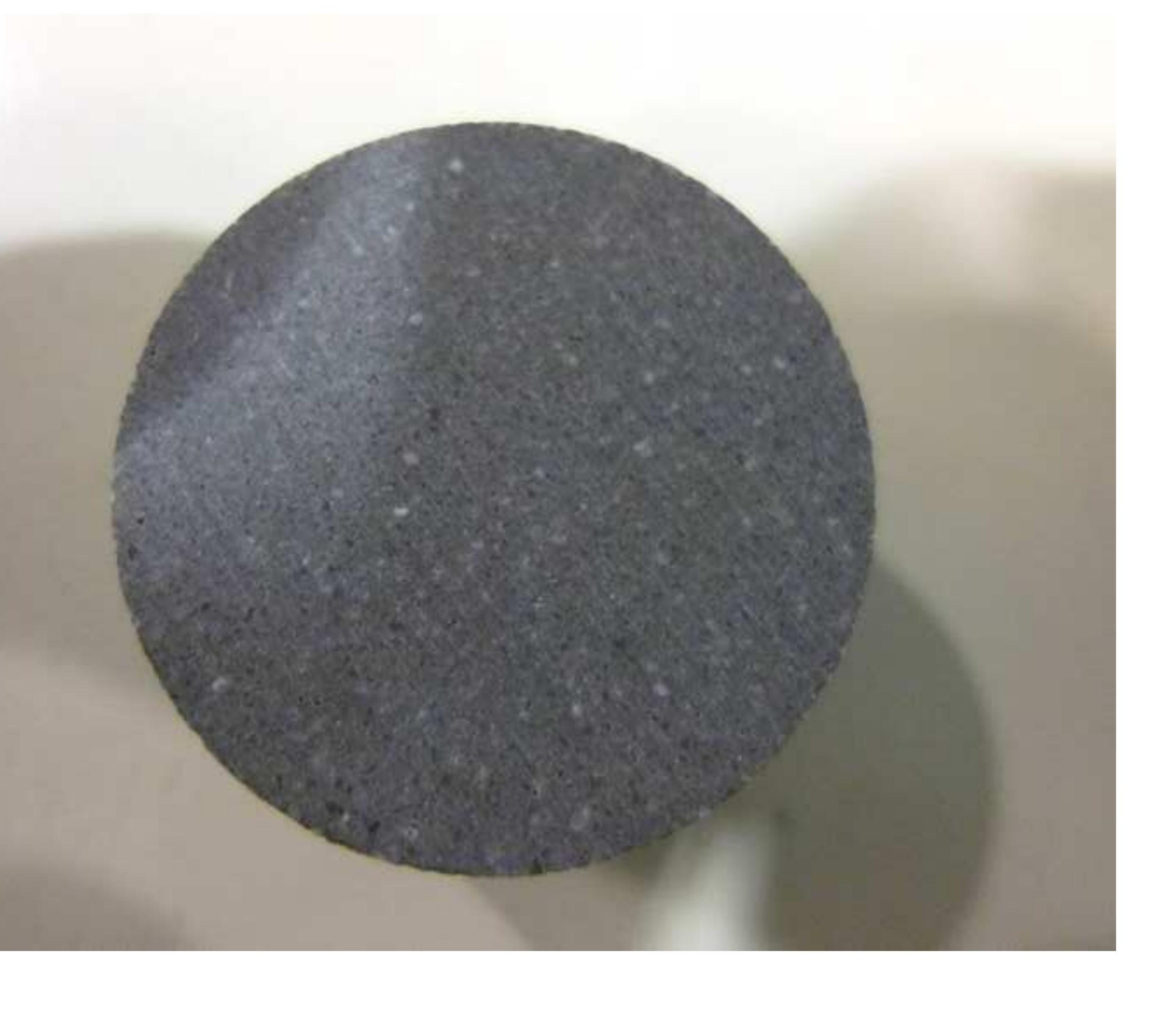


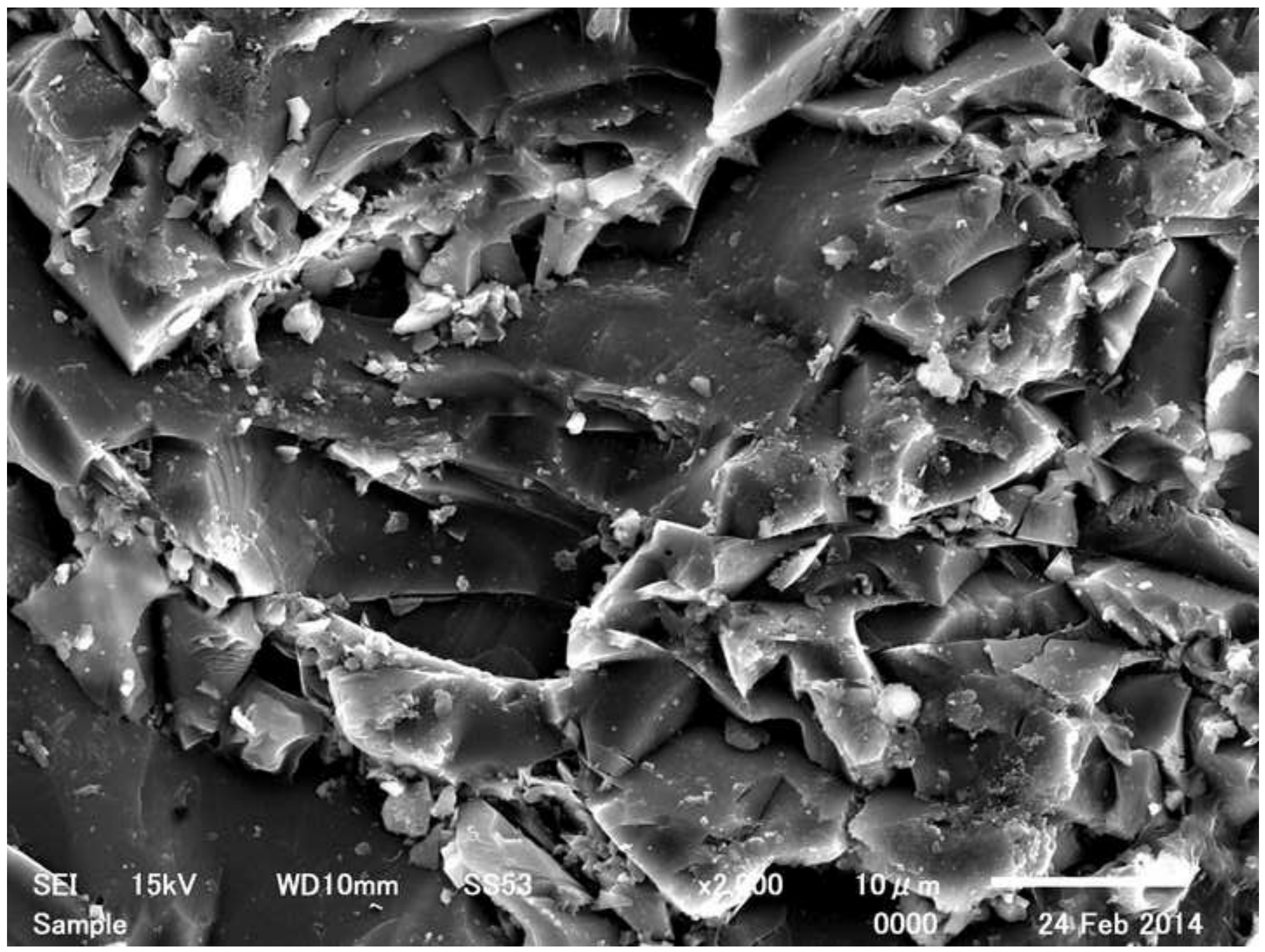




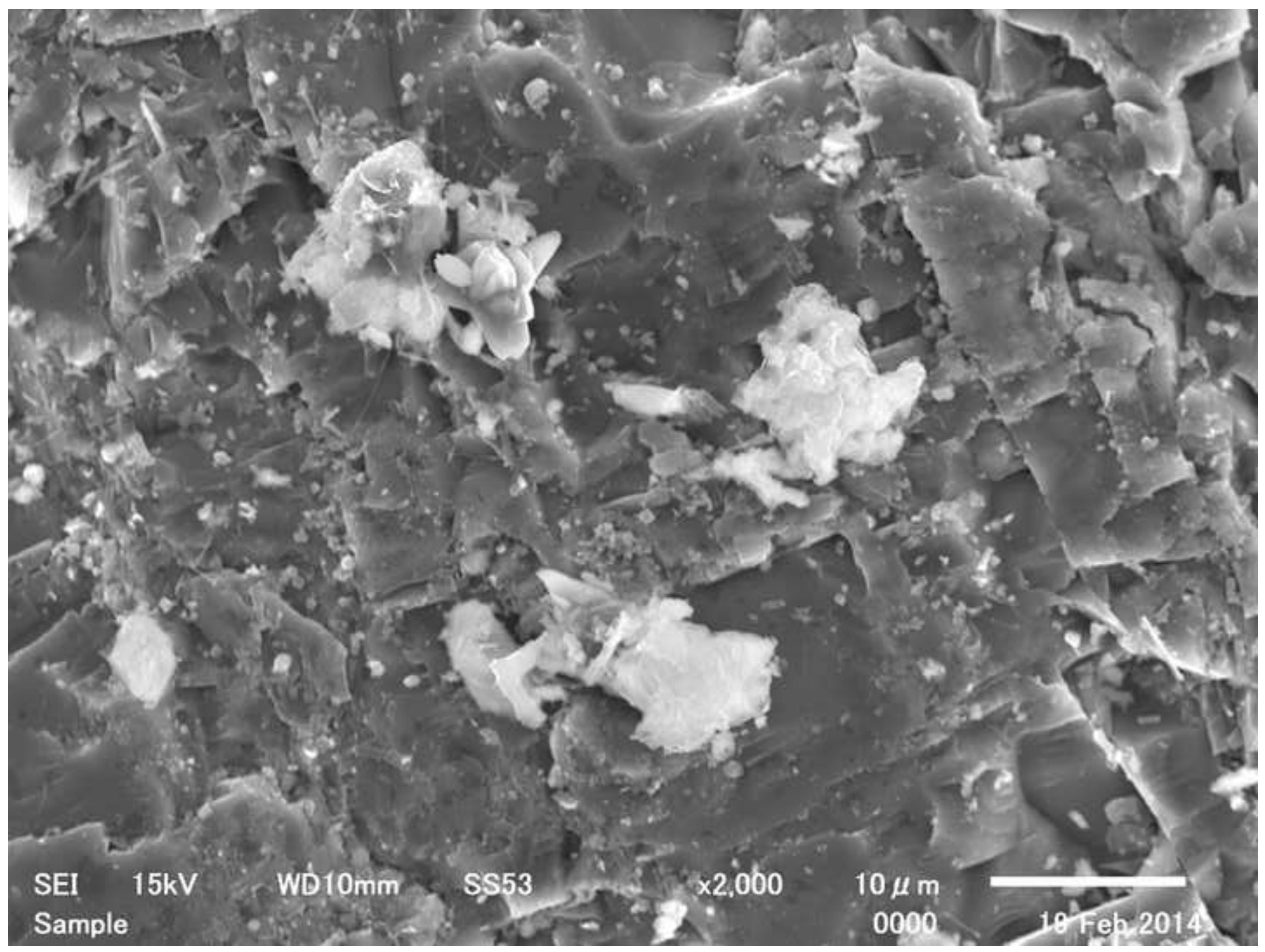




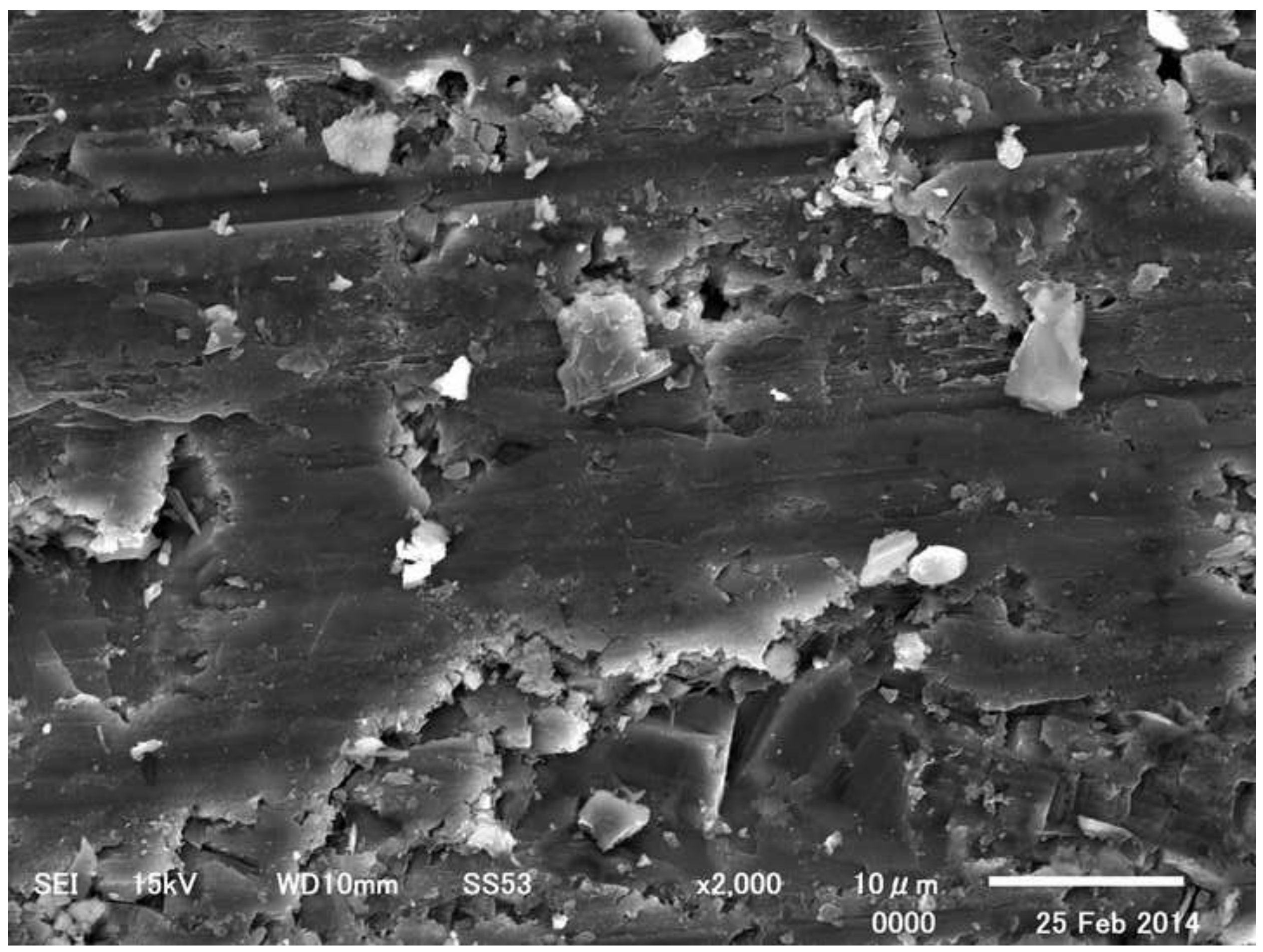




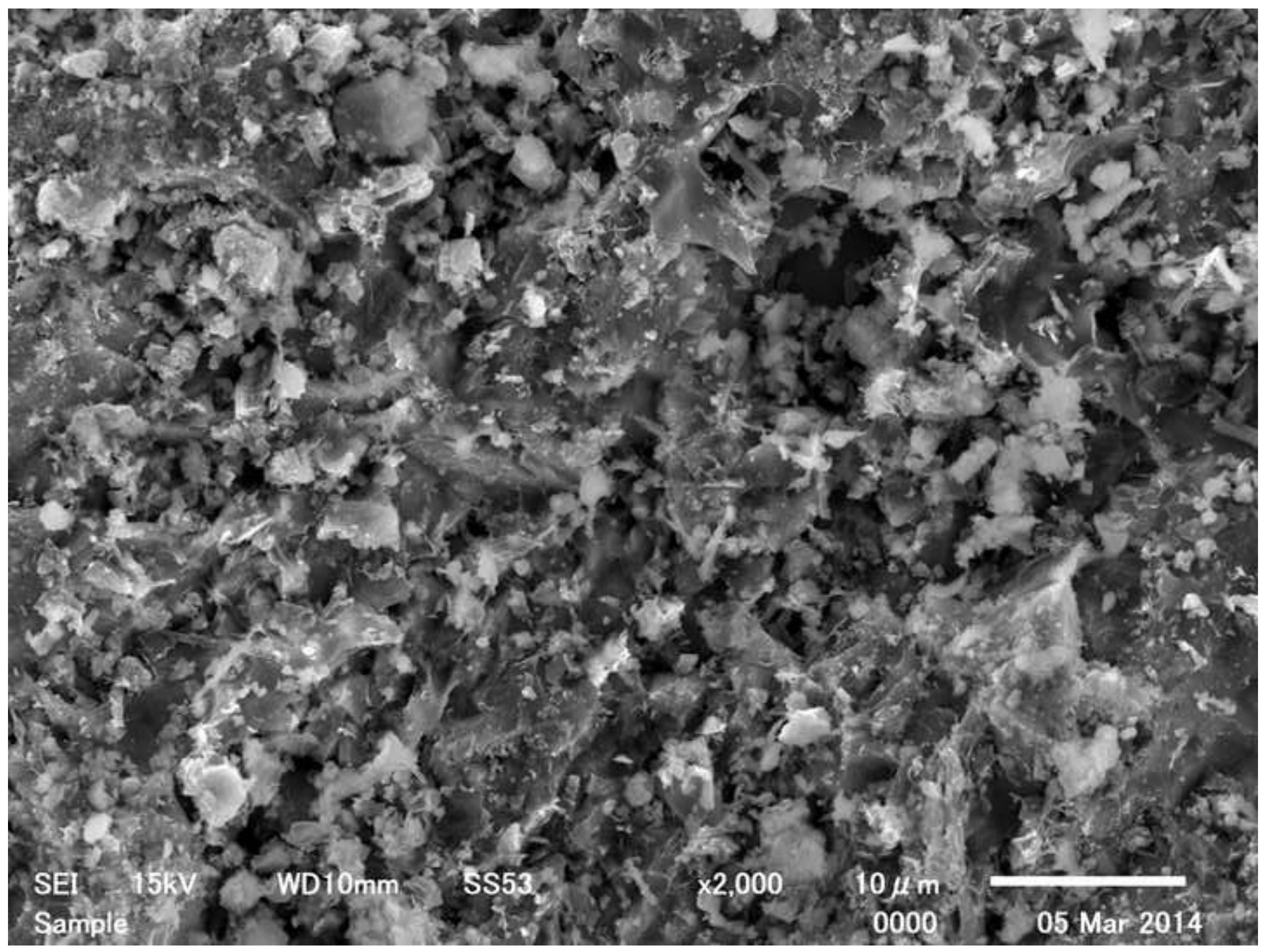




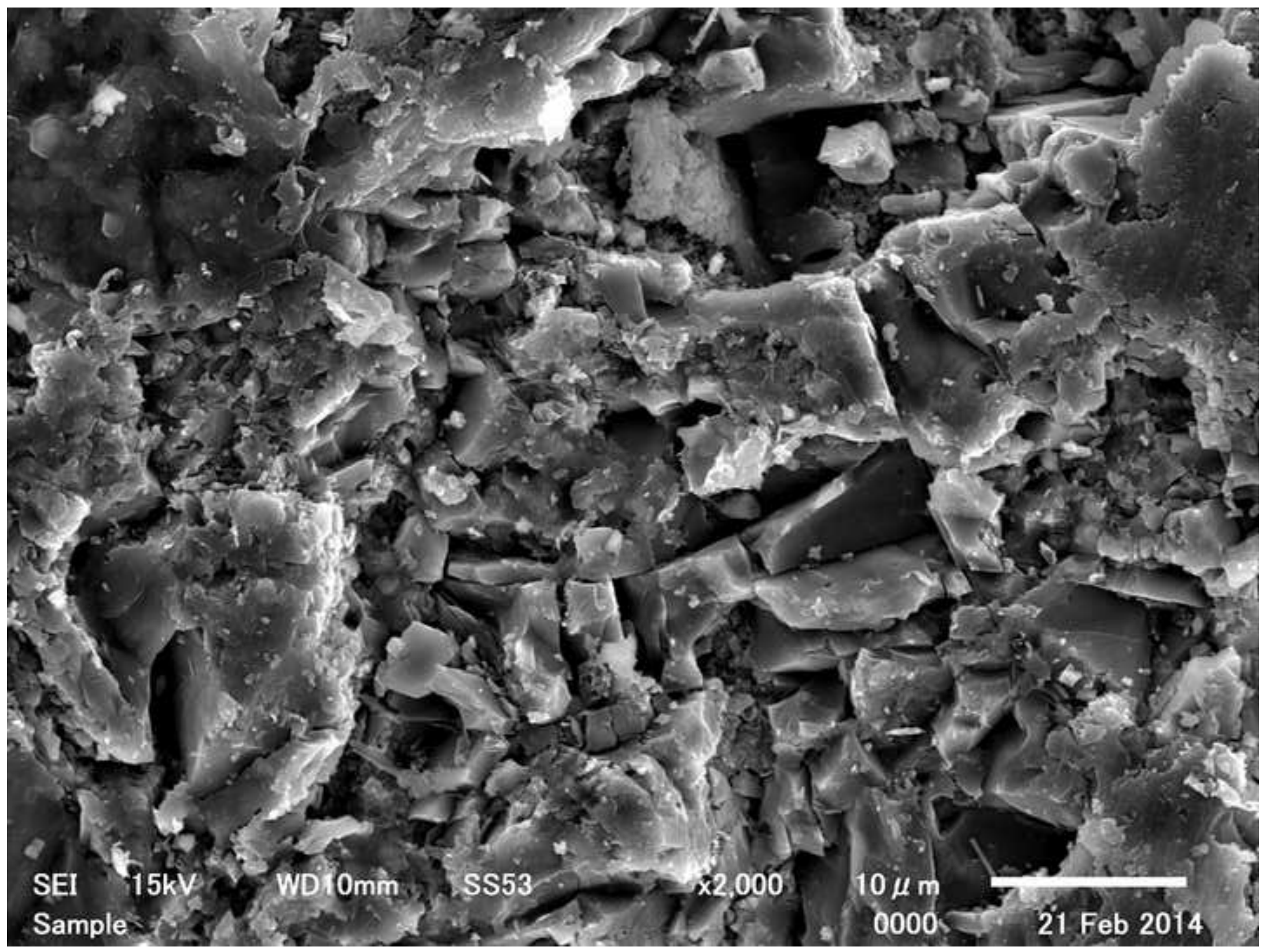




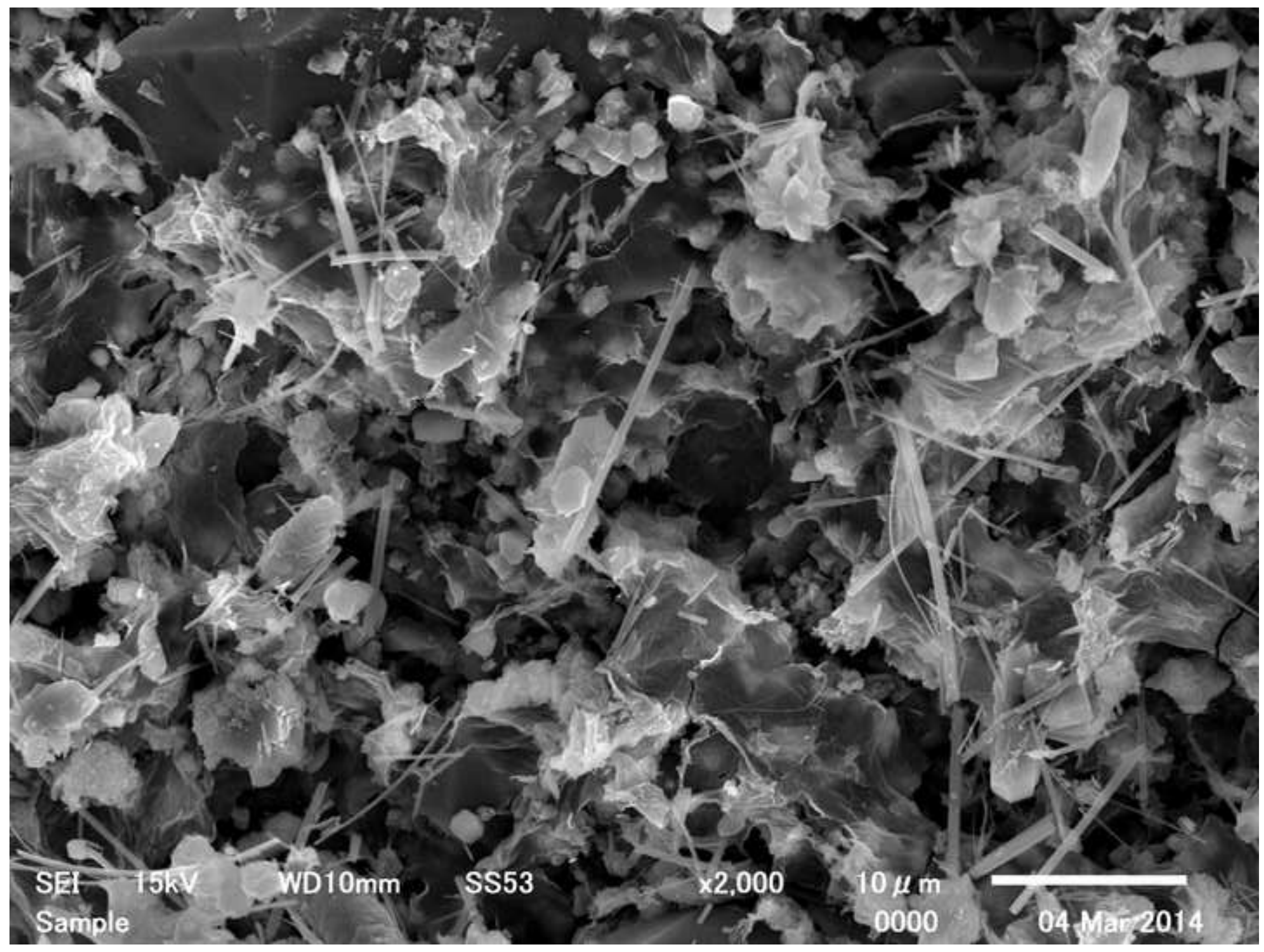




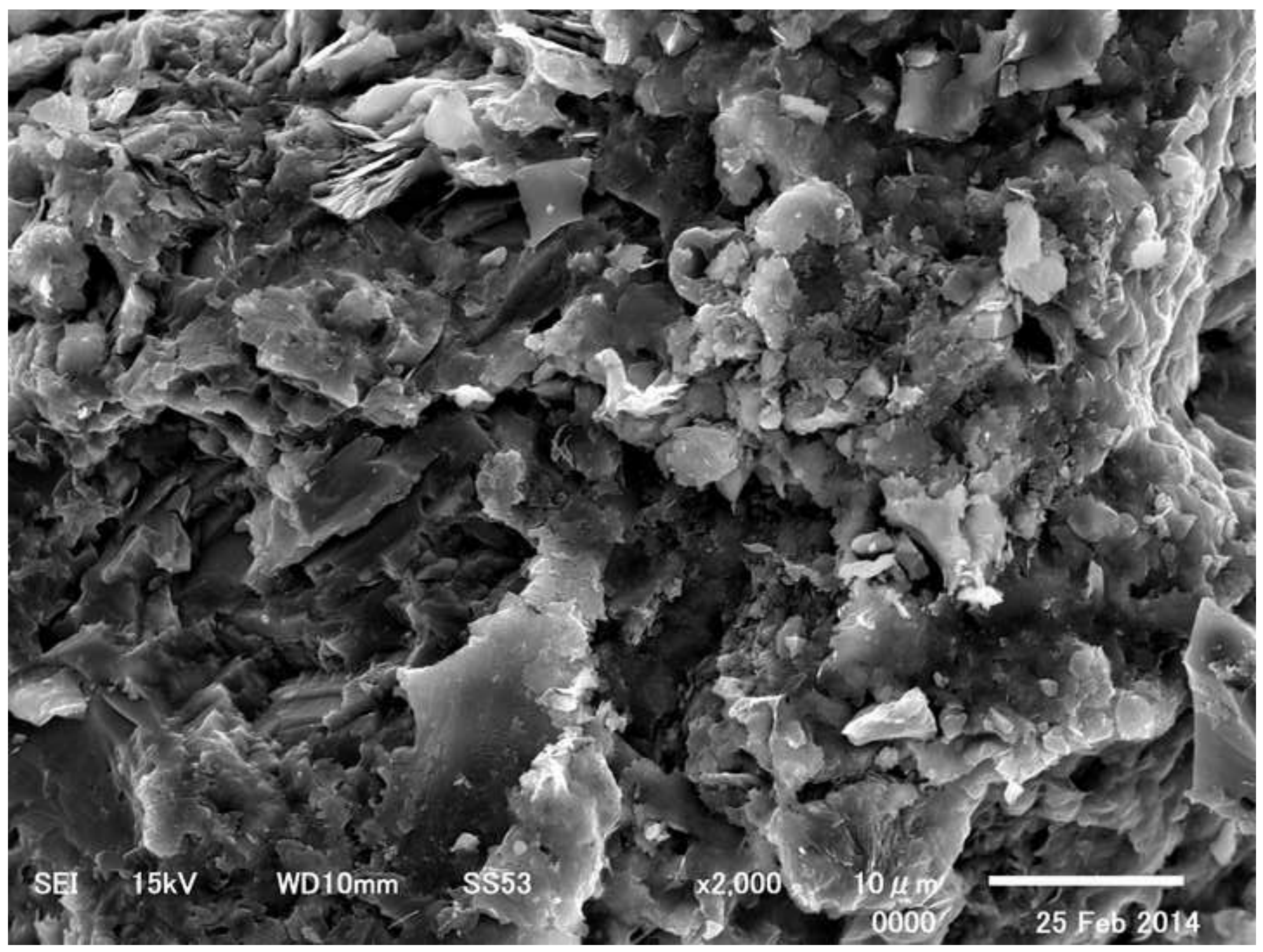




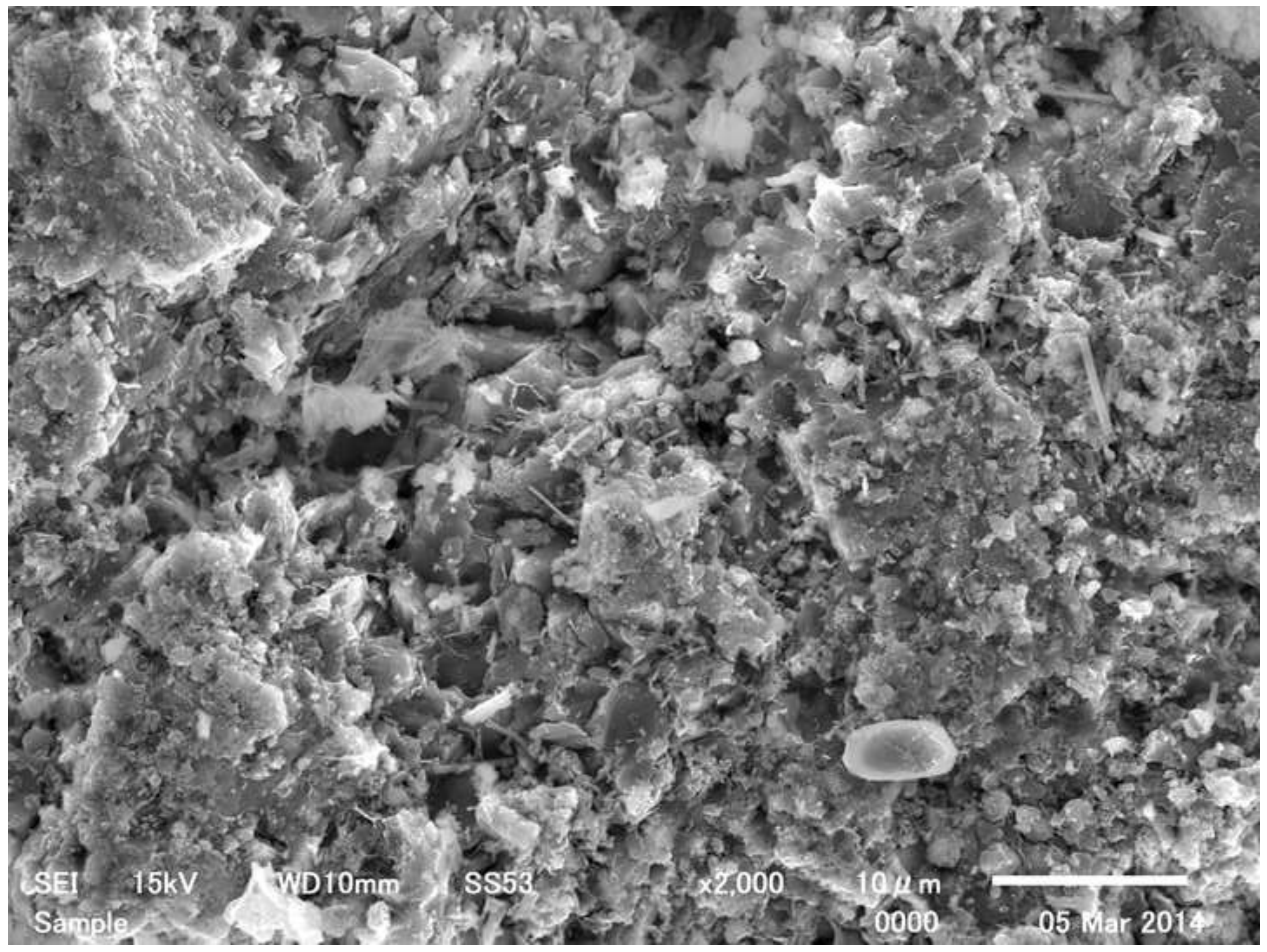




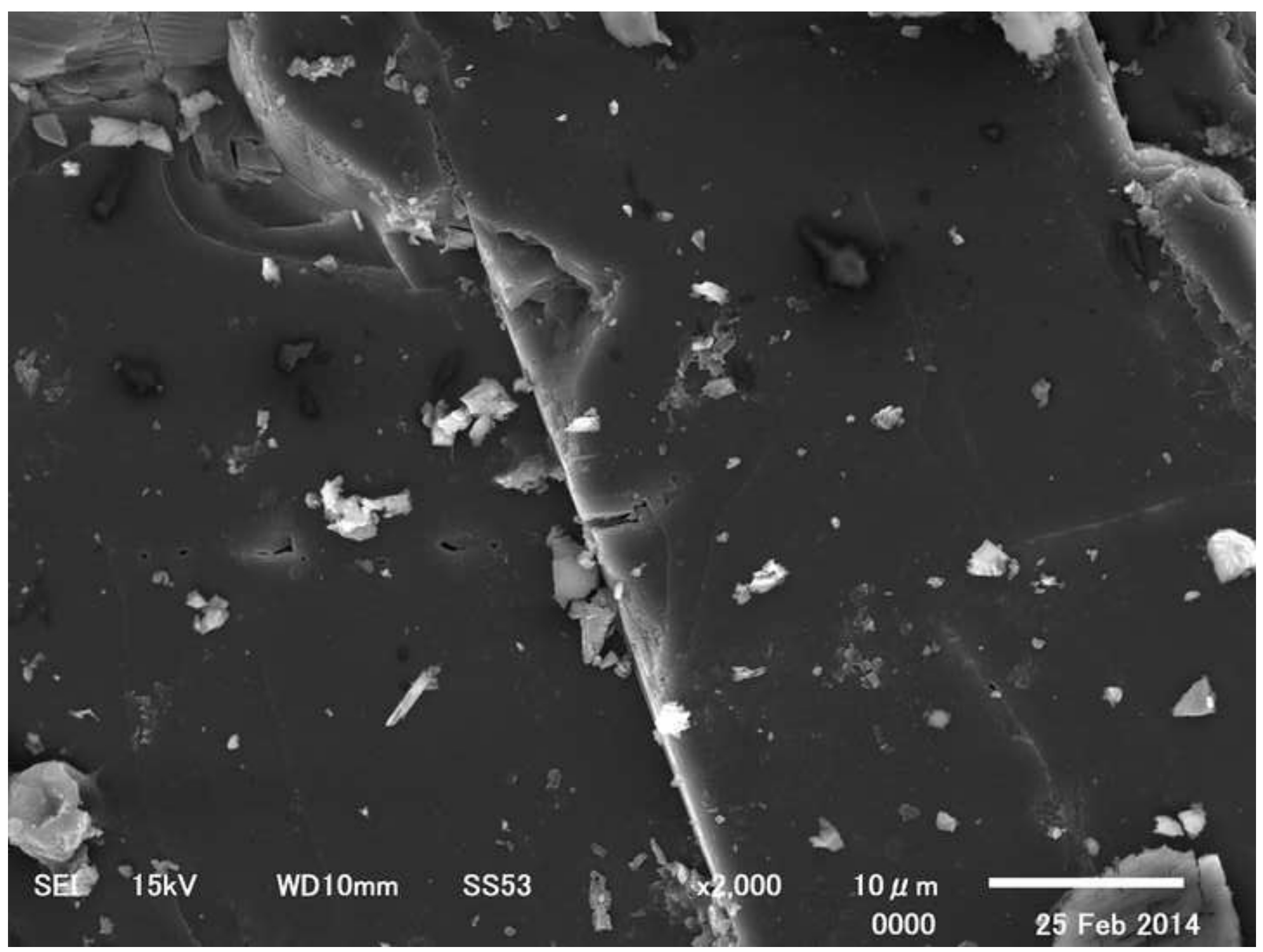




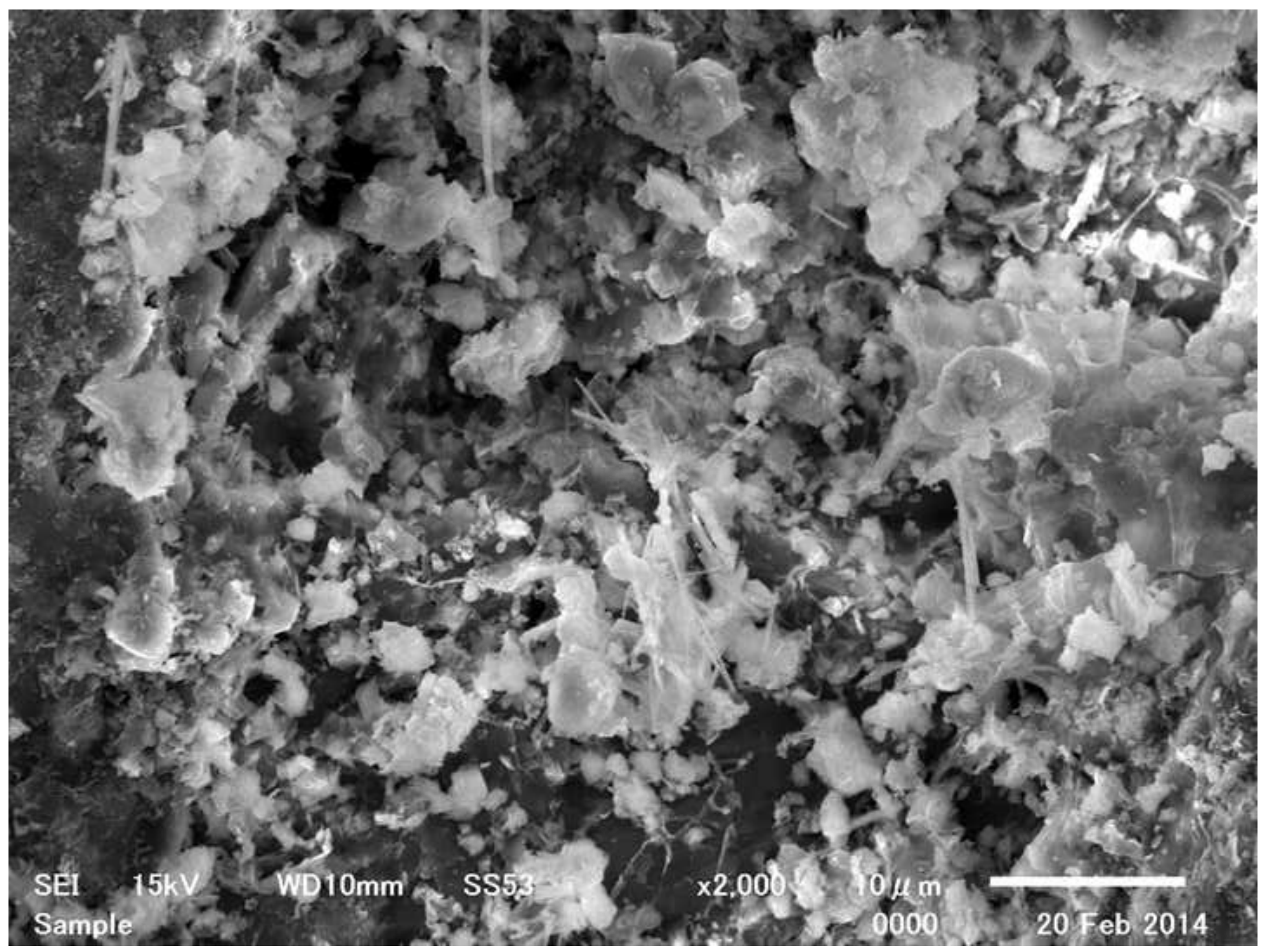

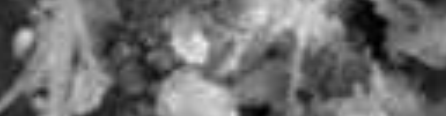

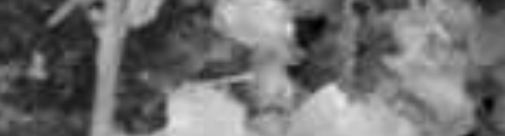

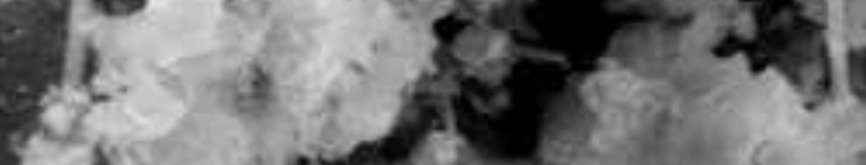

ne

I. 12

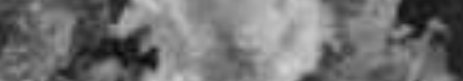

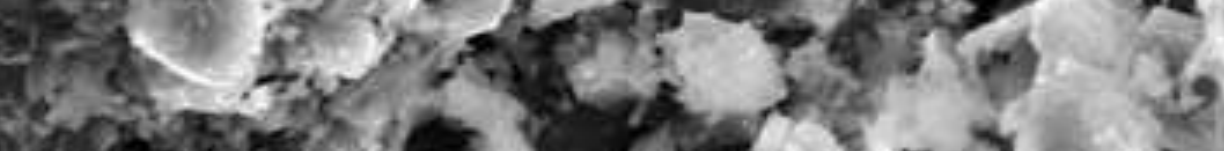

SEL $15 \mathrm{kV} / \mathrm{W}$ - W $10 \mathrm{~mm}$ \& SS5

Sample arsion 


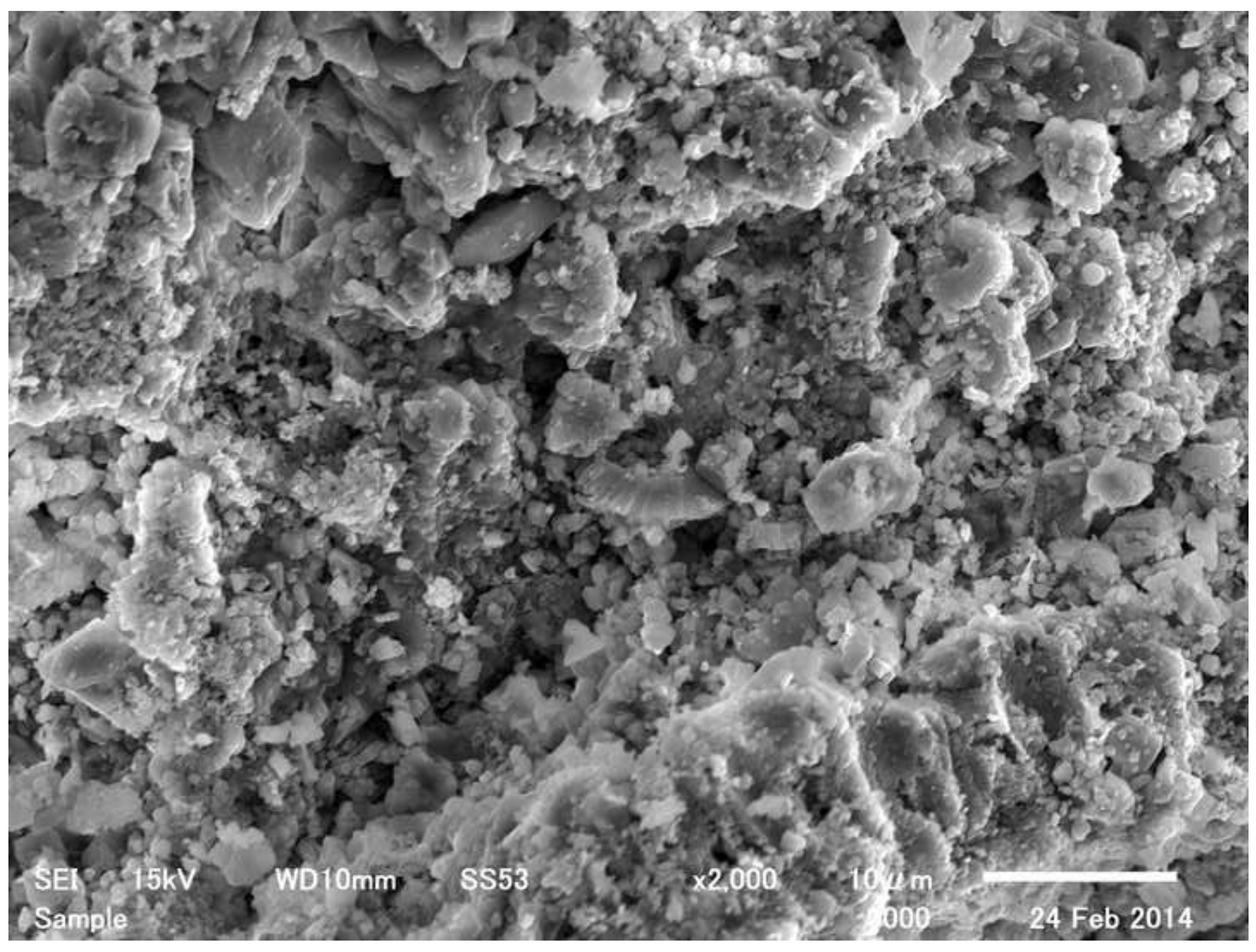

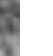

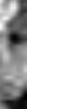

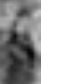

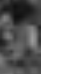

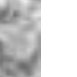

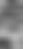

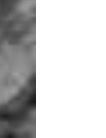

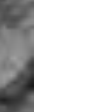

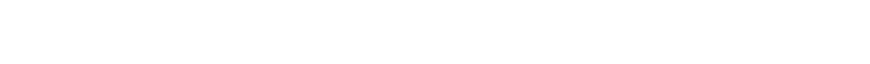

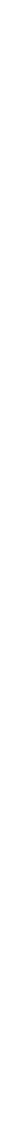

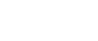




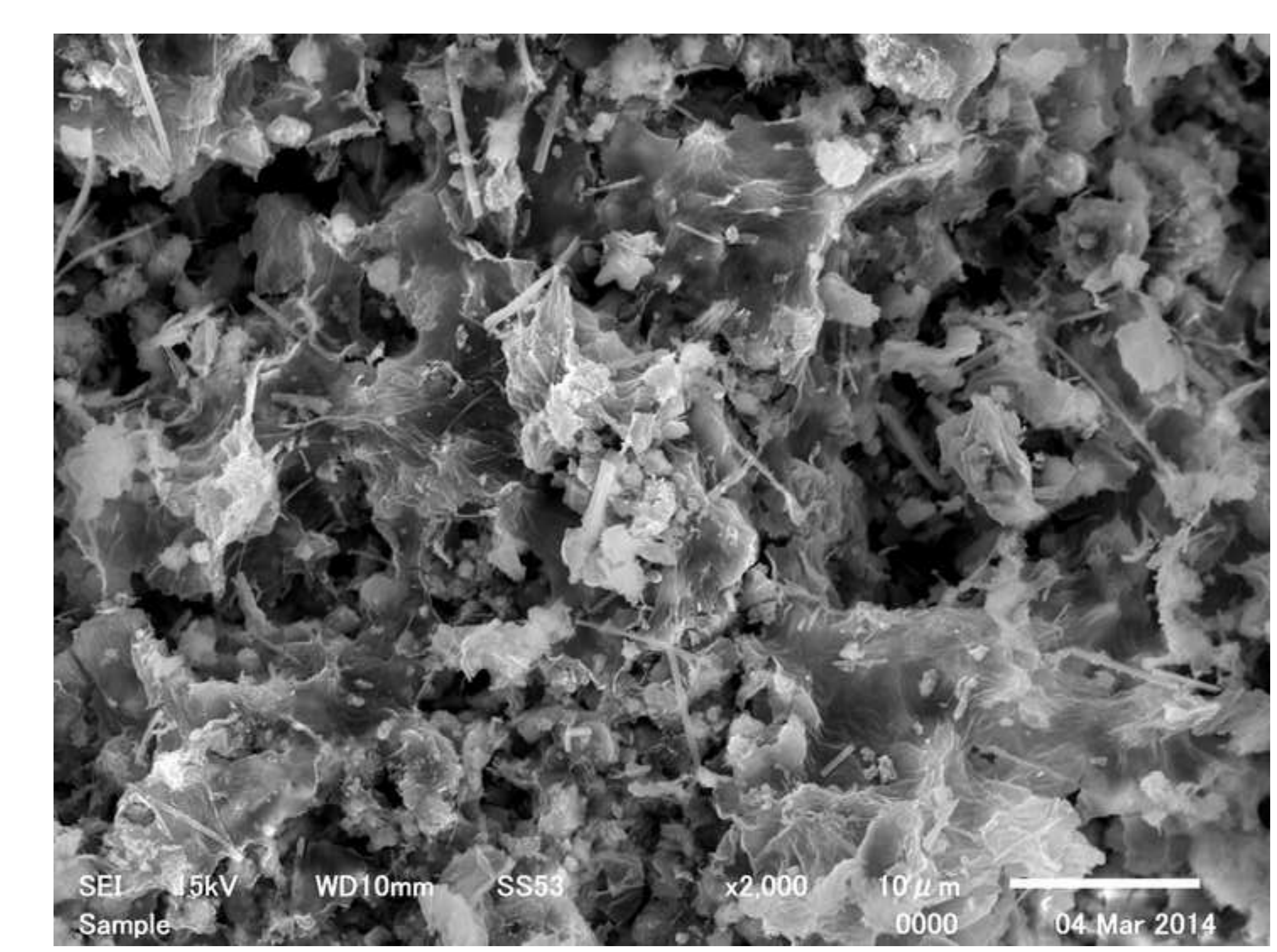

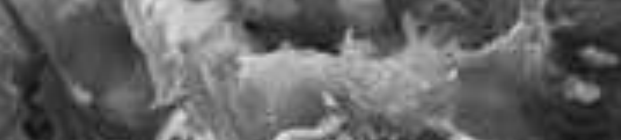

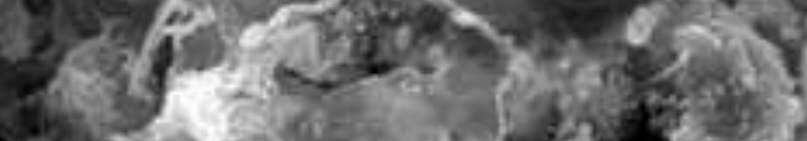

19

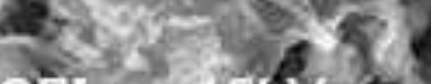

Sample $5 \mathrm{kV}$. W

$5 S 58=2 \times 2000$ 0000

04Mar 2014 


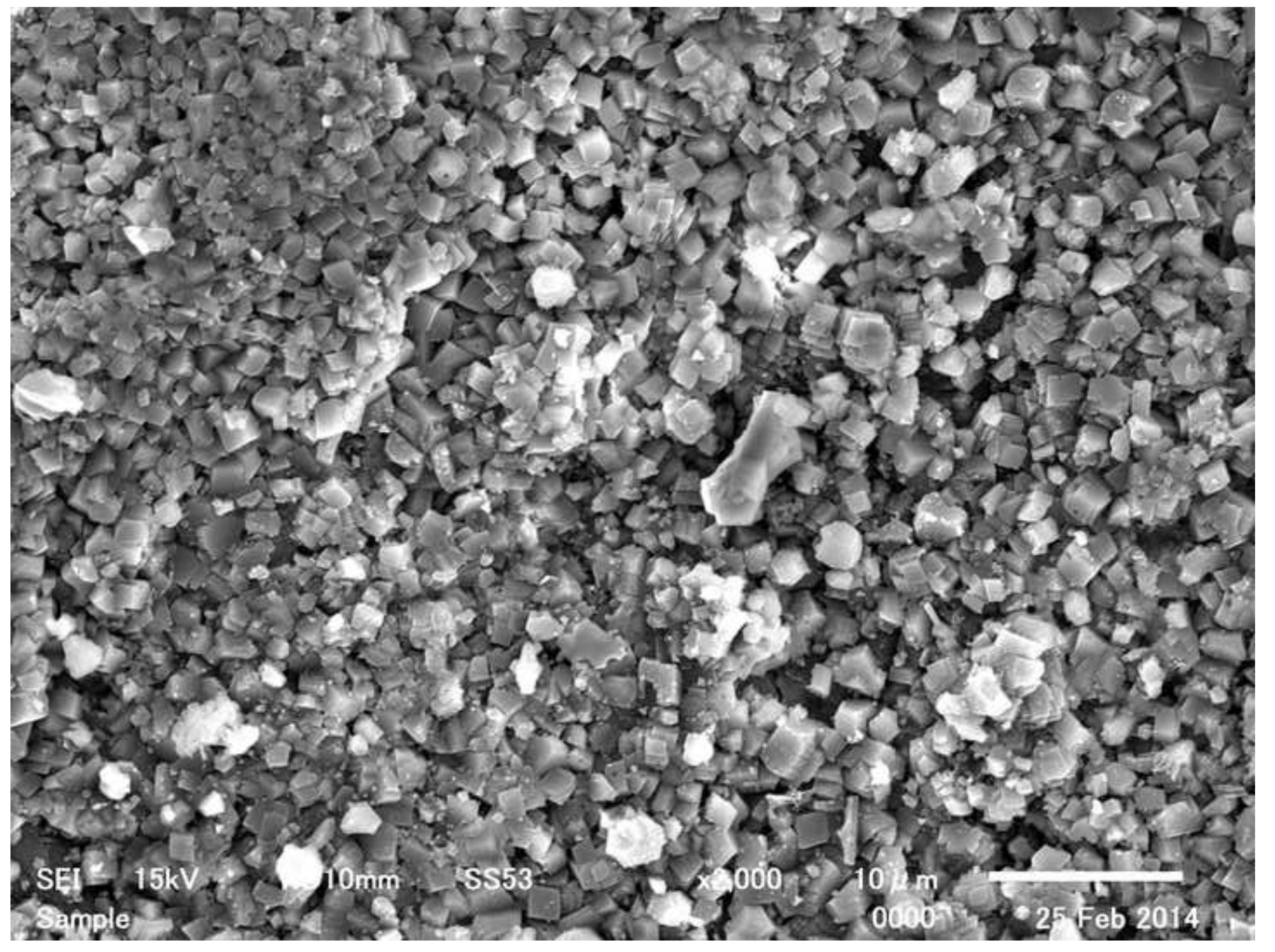




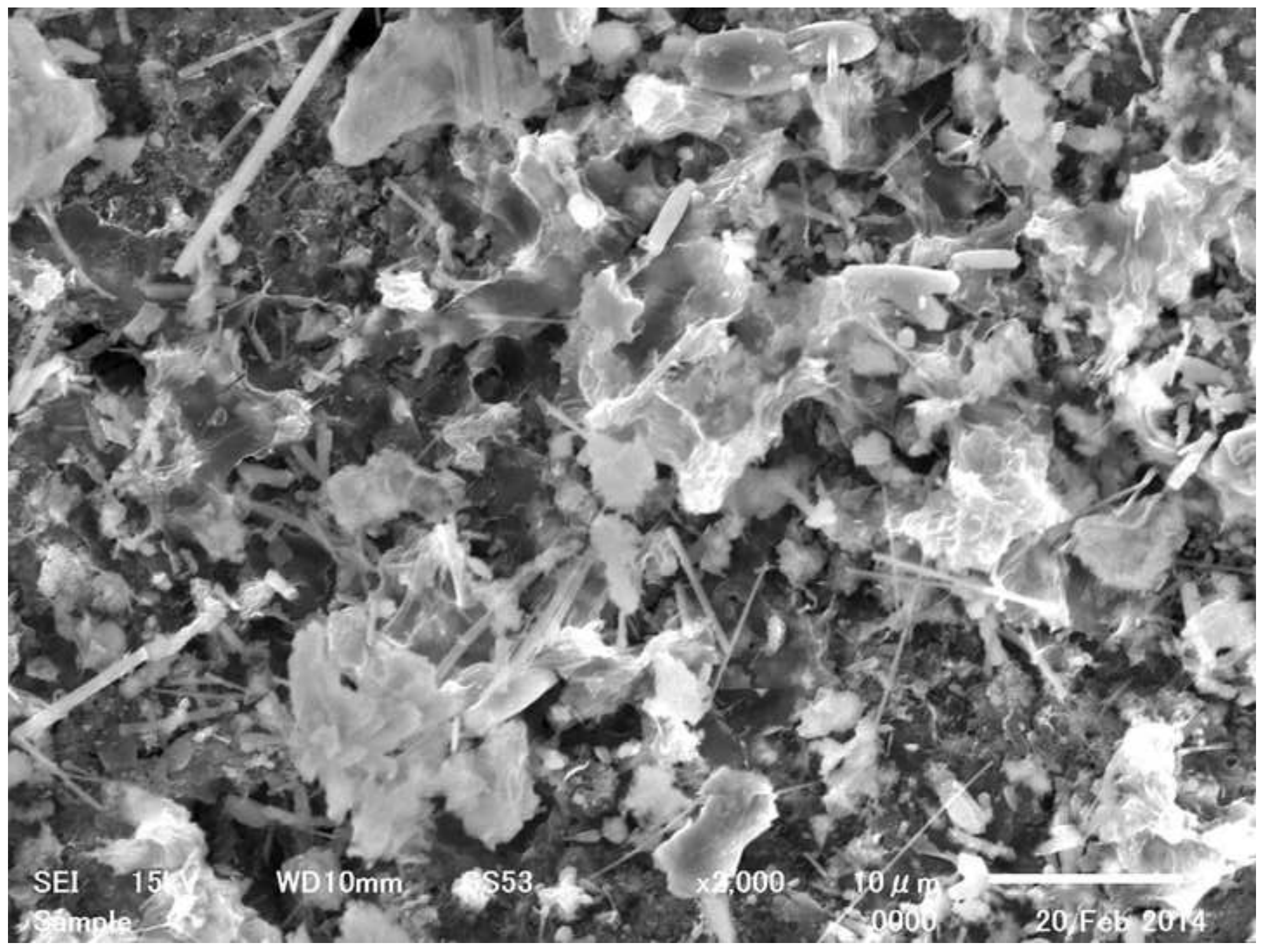


Figure $8 a$

Click here to download Figure Fig_08a(BS_HSULPC_powder).jpg $\stackrel{ \pm}{ }$
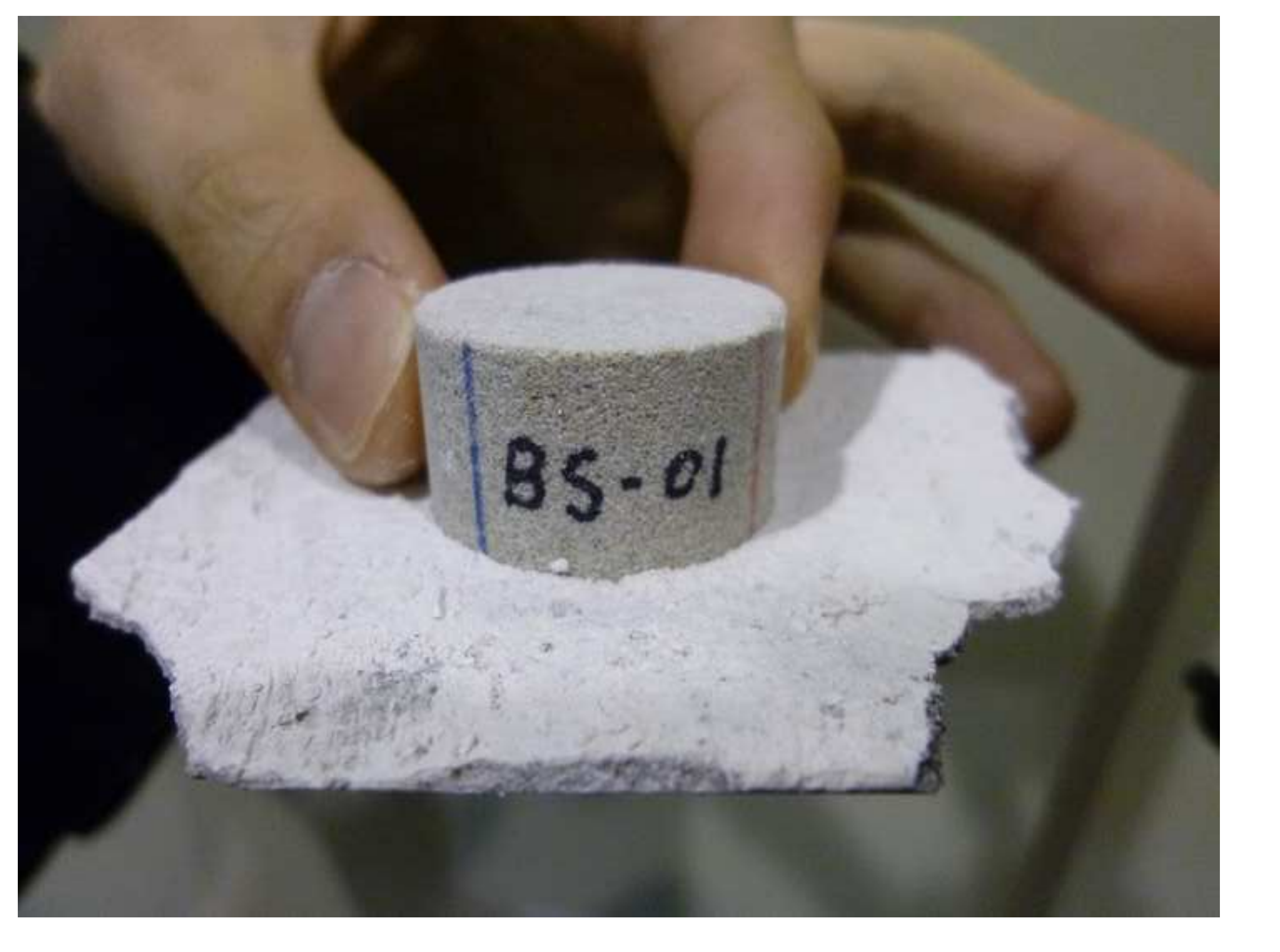

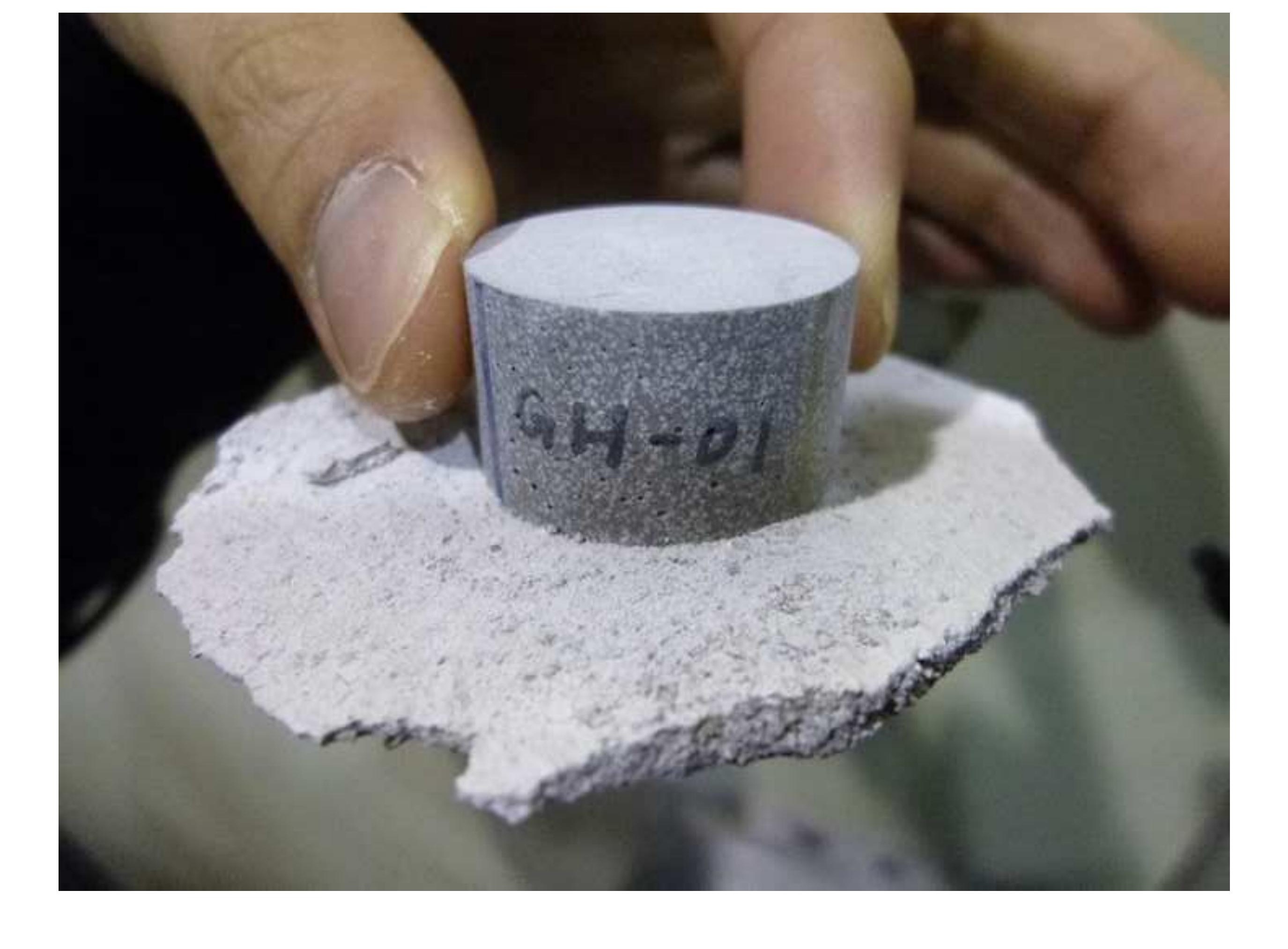


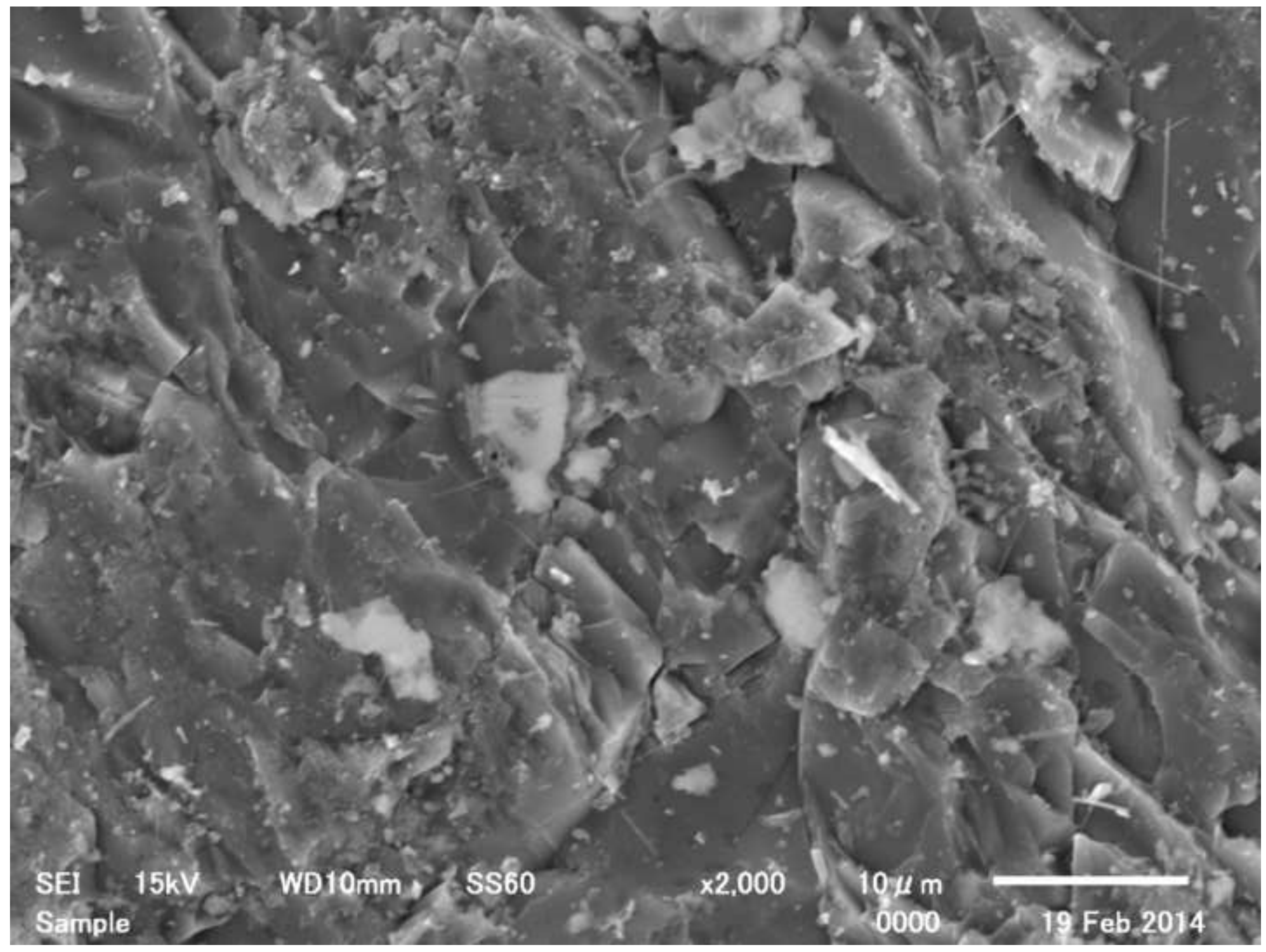




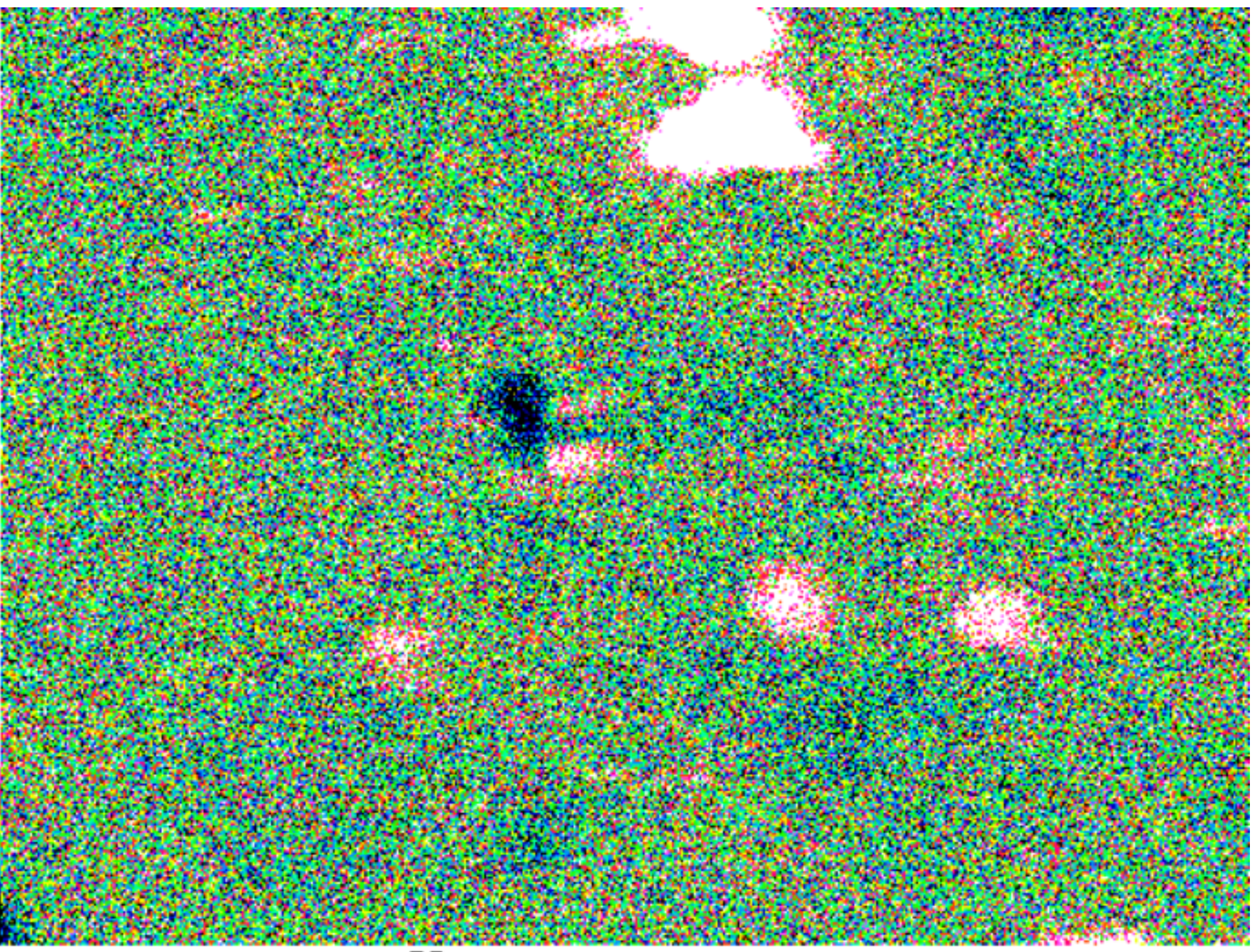


W $20 \mu \mathrm{m}$ 
W

50 m.

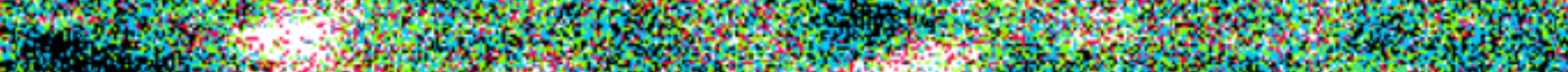
H.

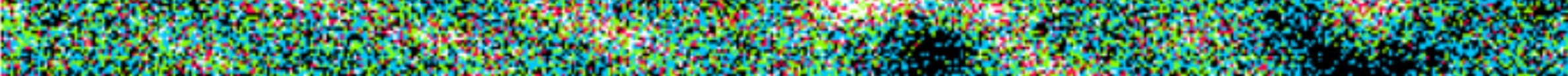

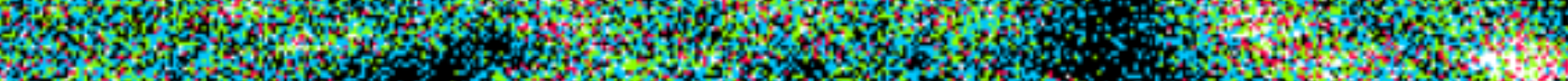

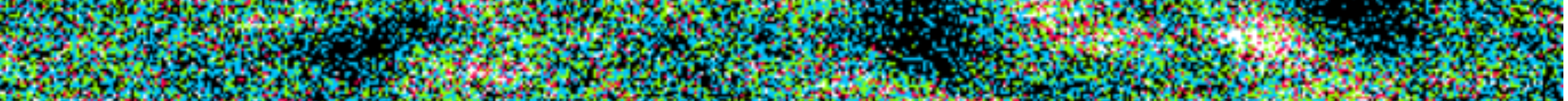

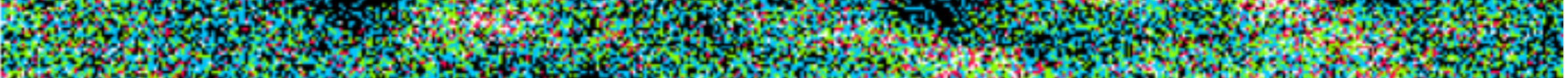
1.5.

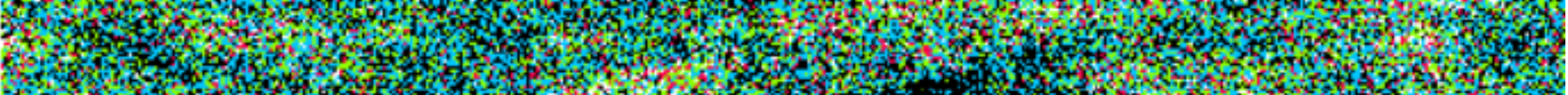

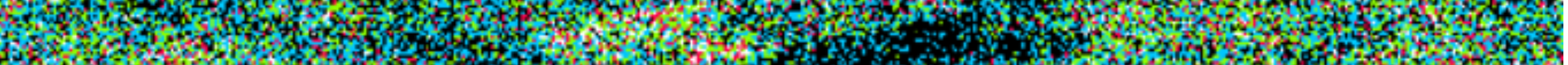

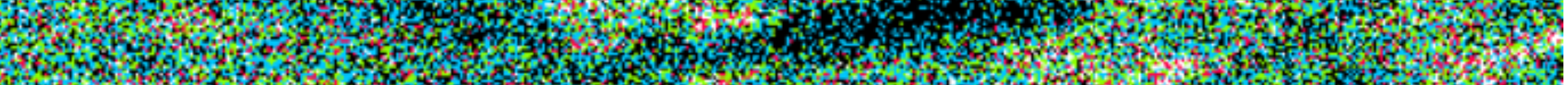

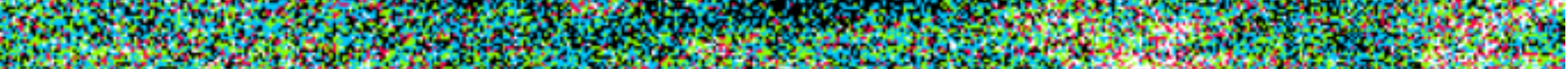

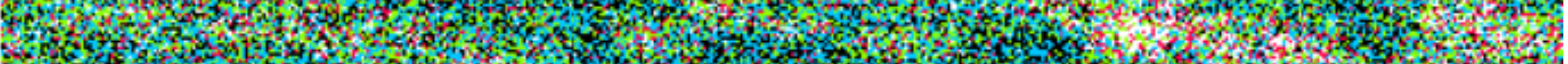

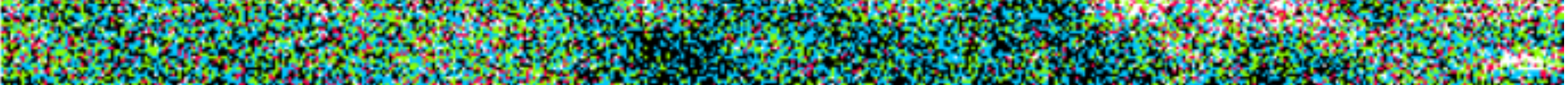
W.

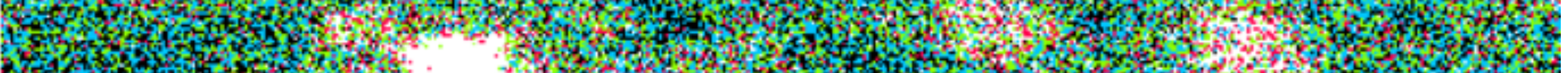

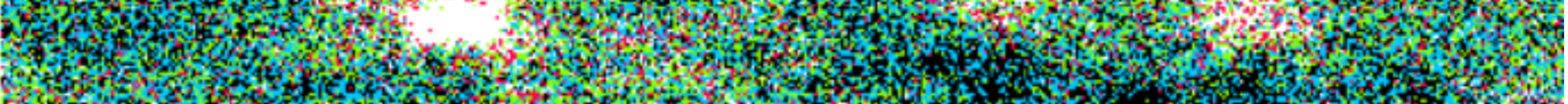

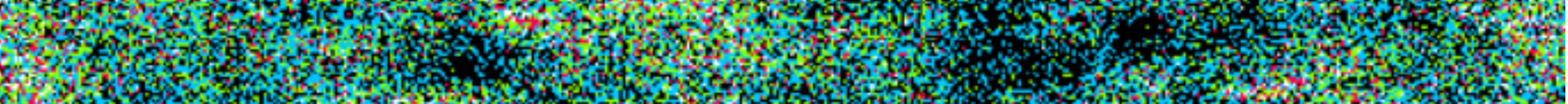

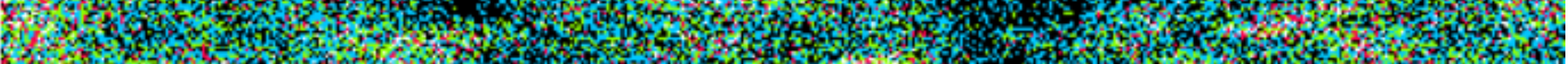

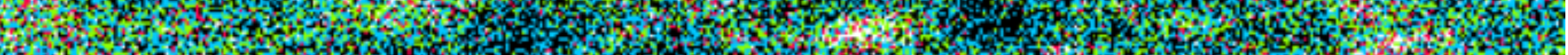

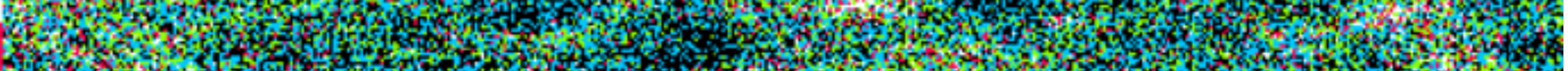

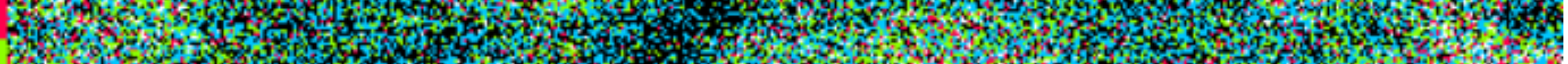

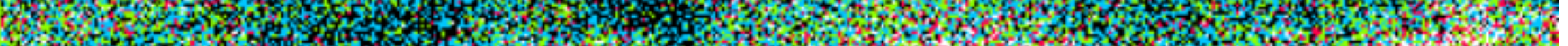

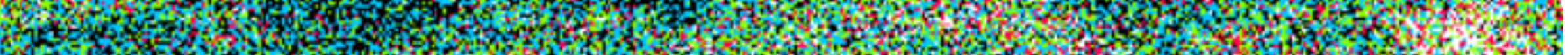

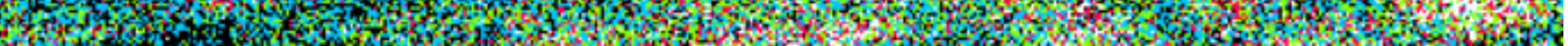
3.

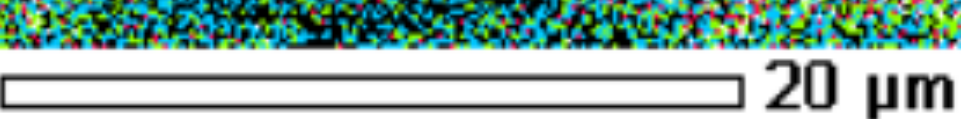

C K 


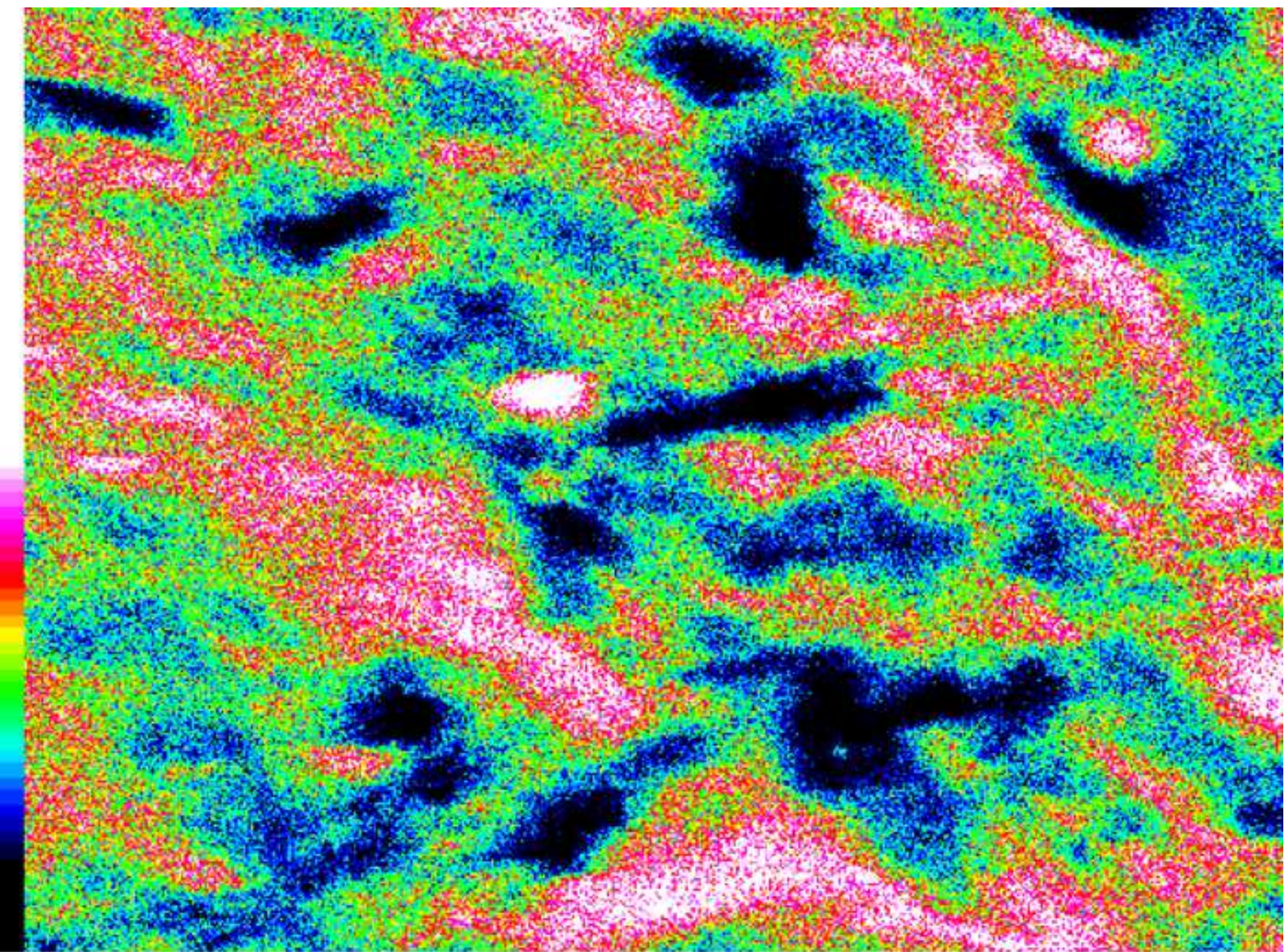




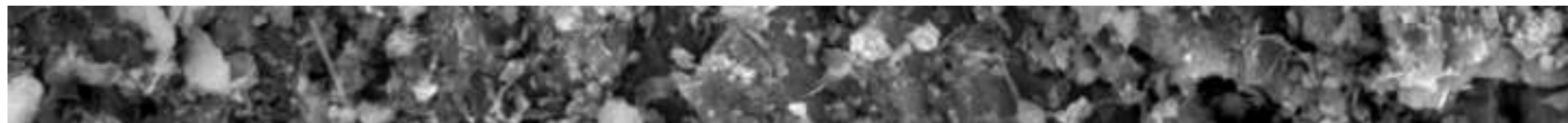

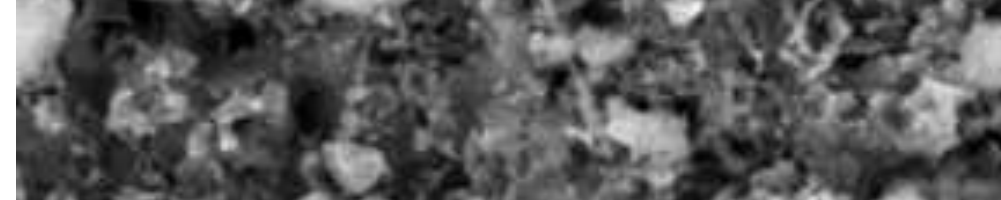

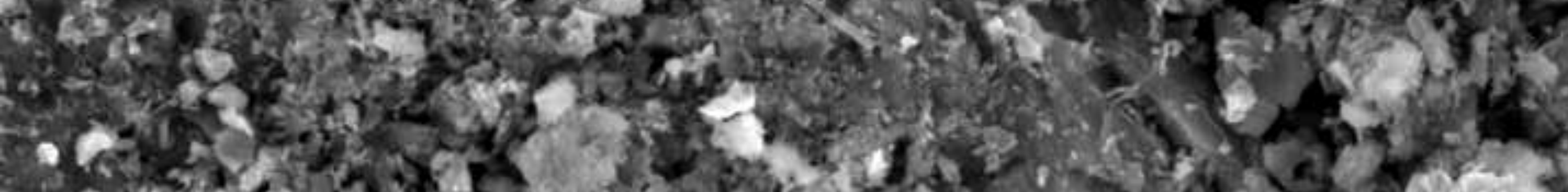

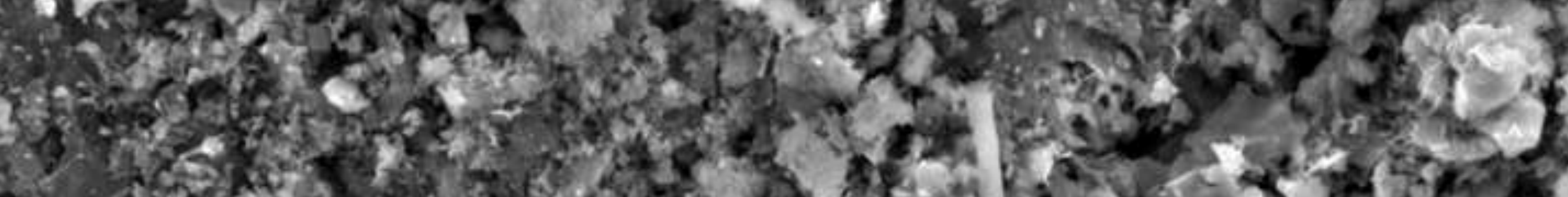
19. 1 .

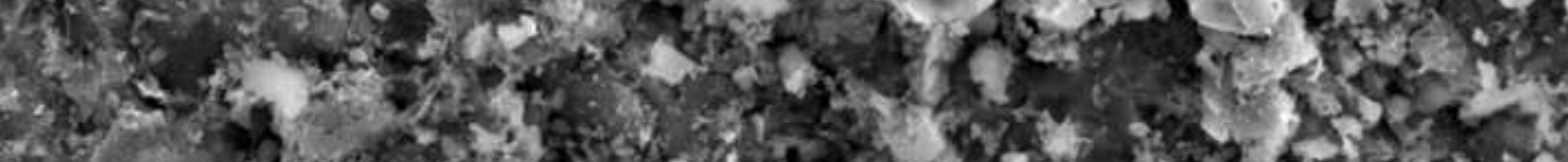

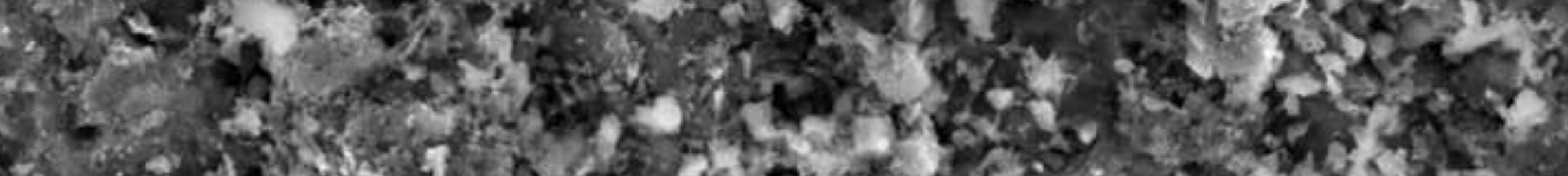

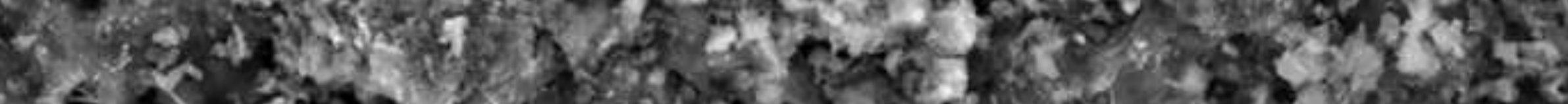
5.

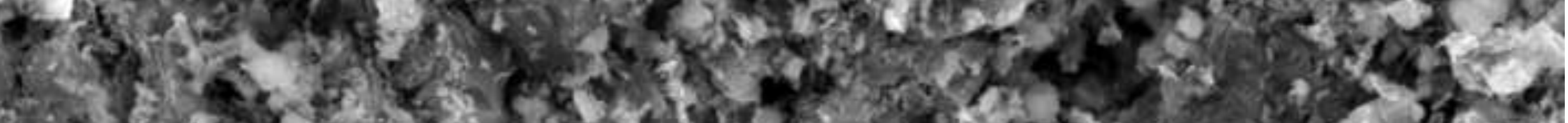

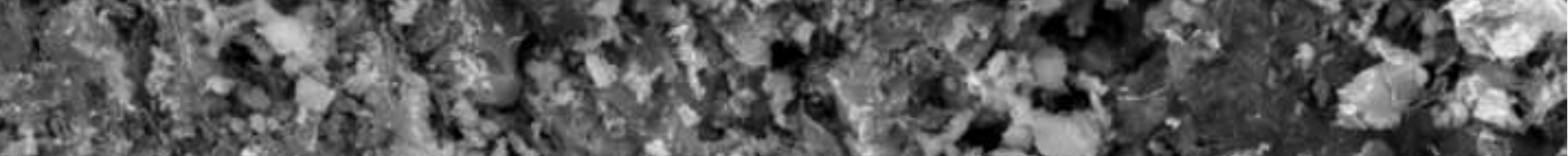
5. (2)

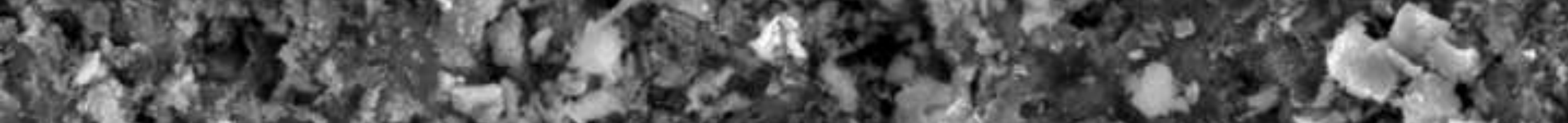




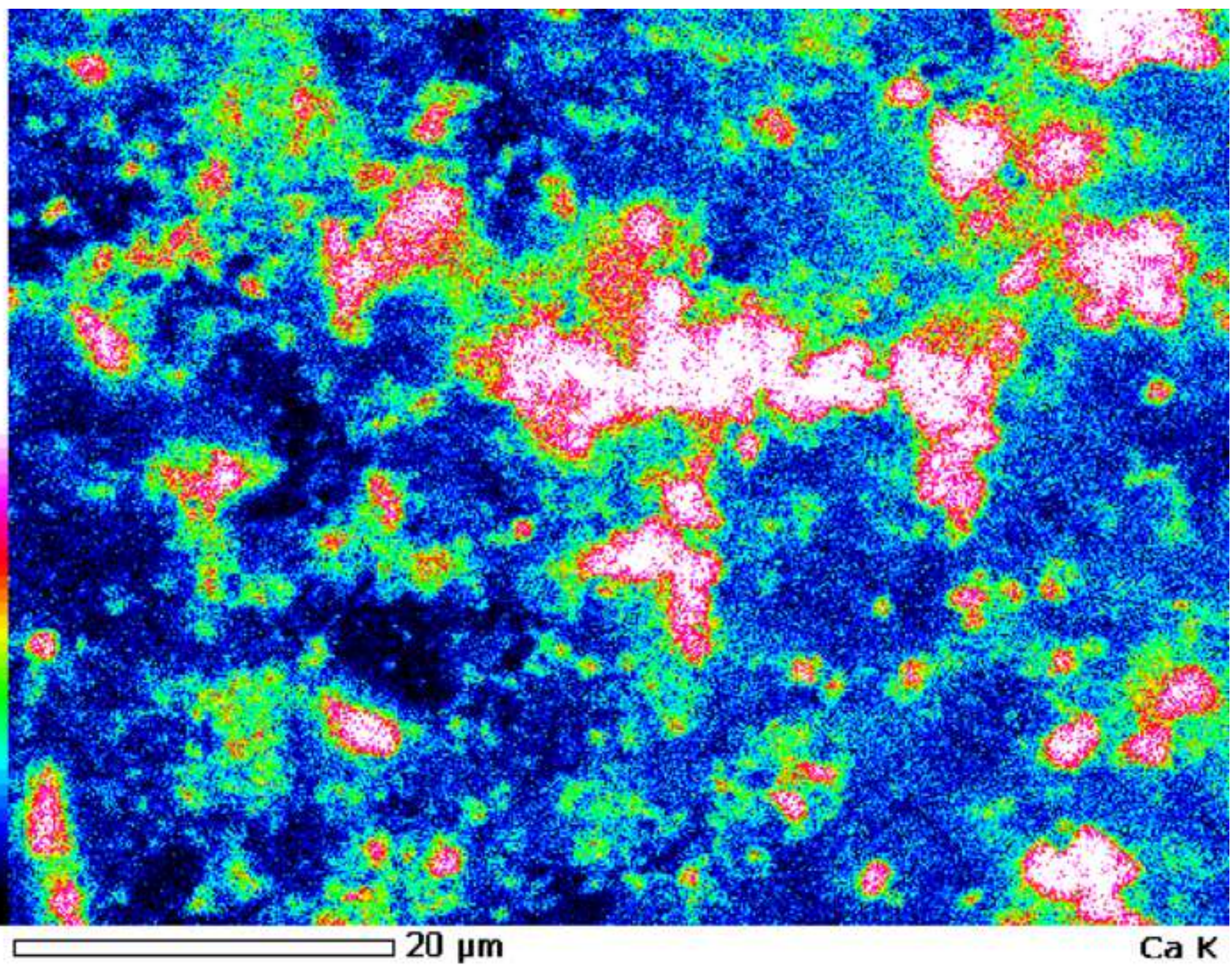




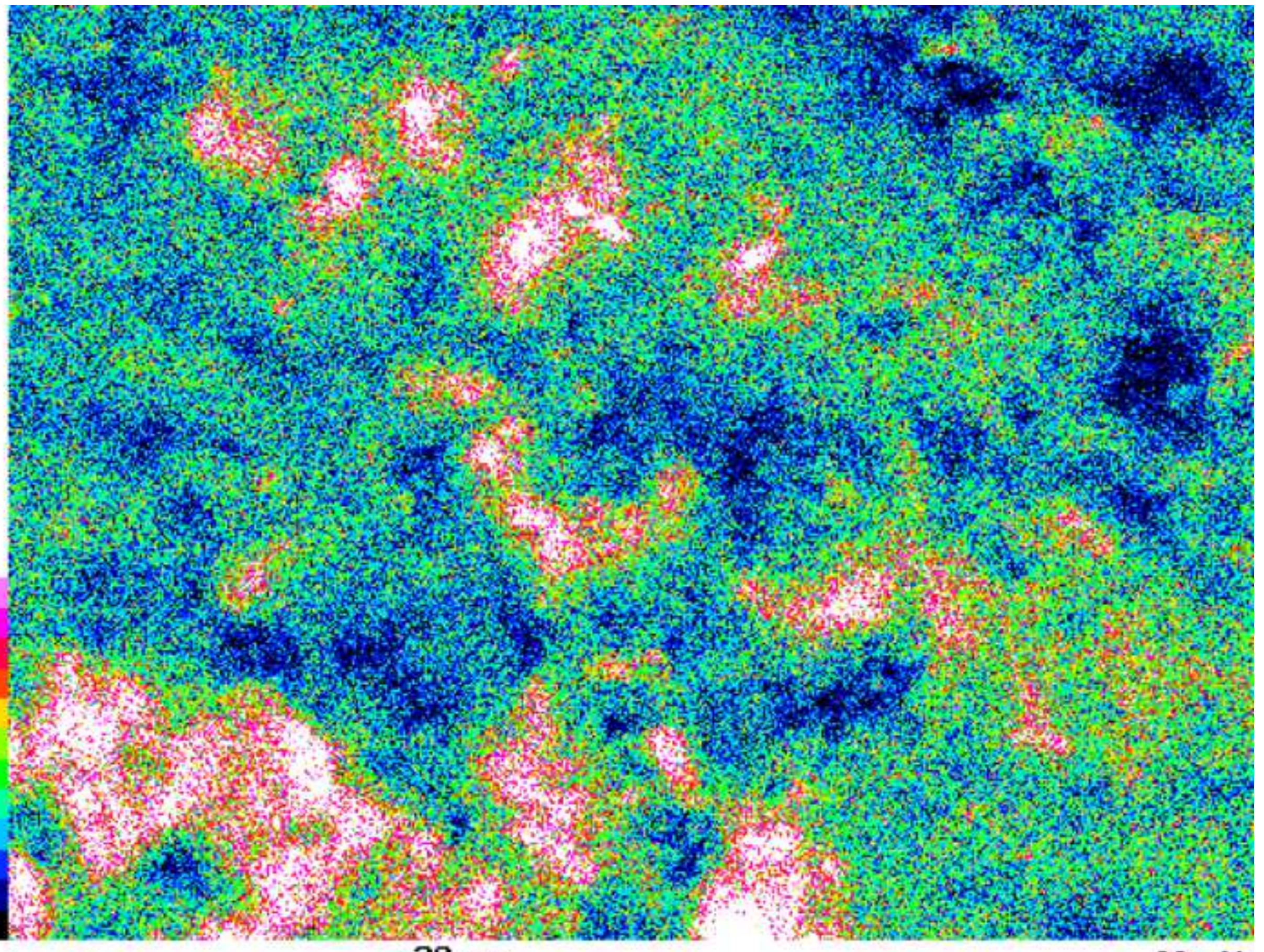




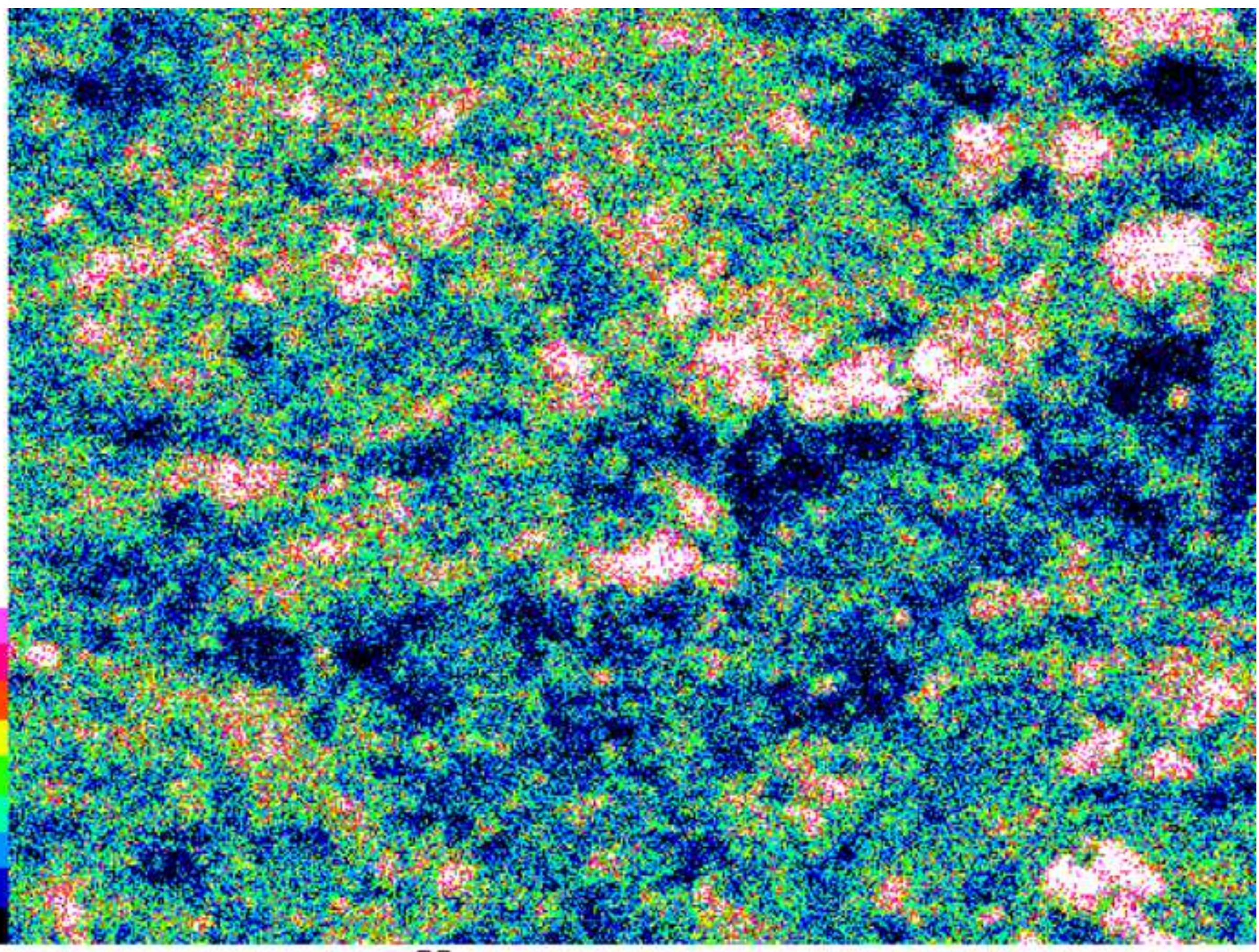




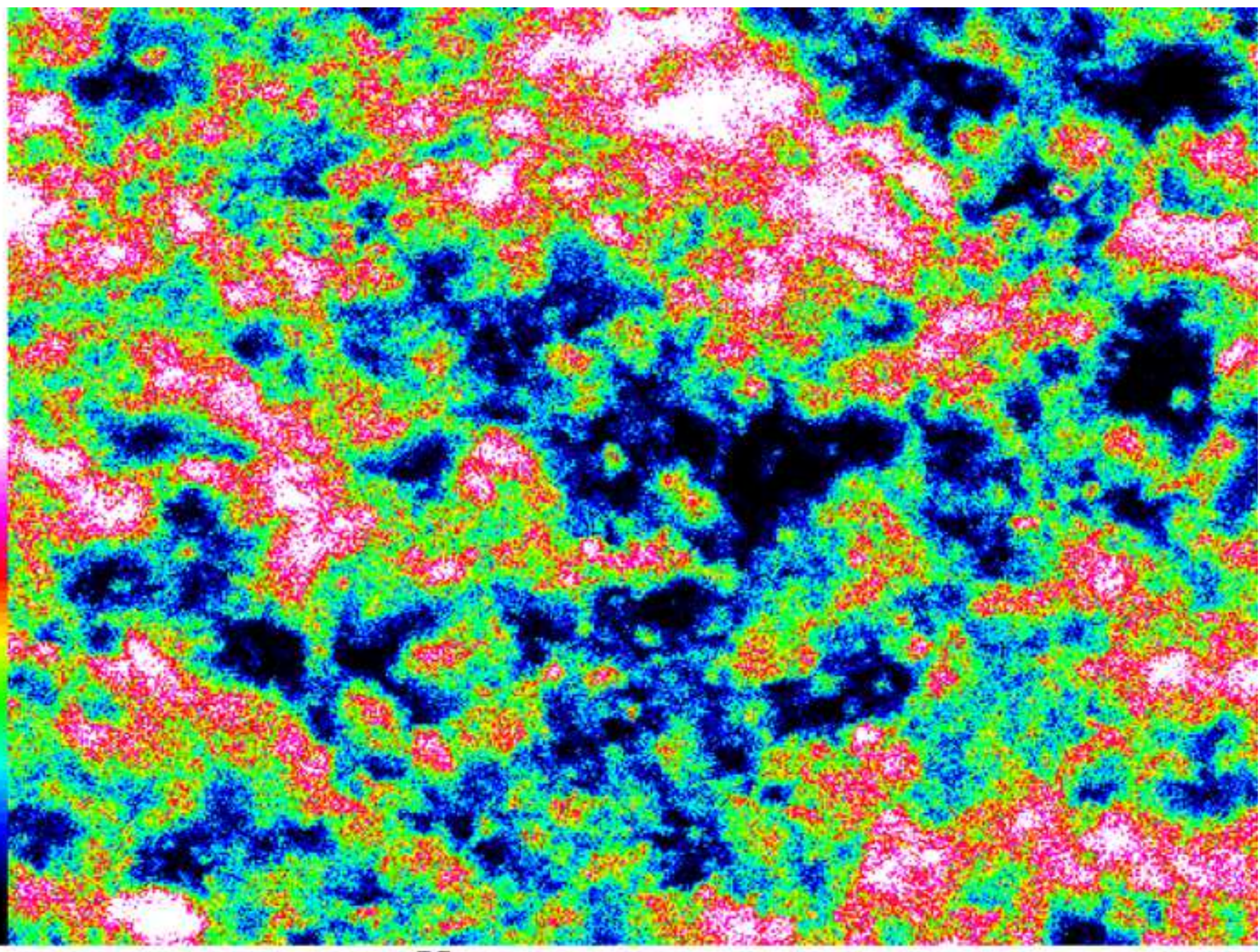




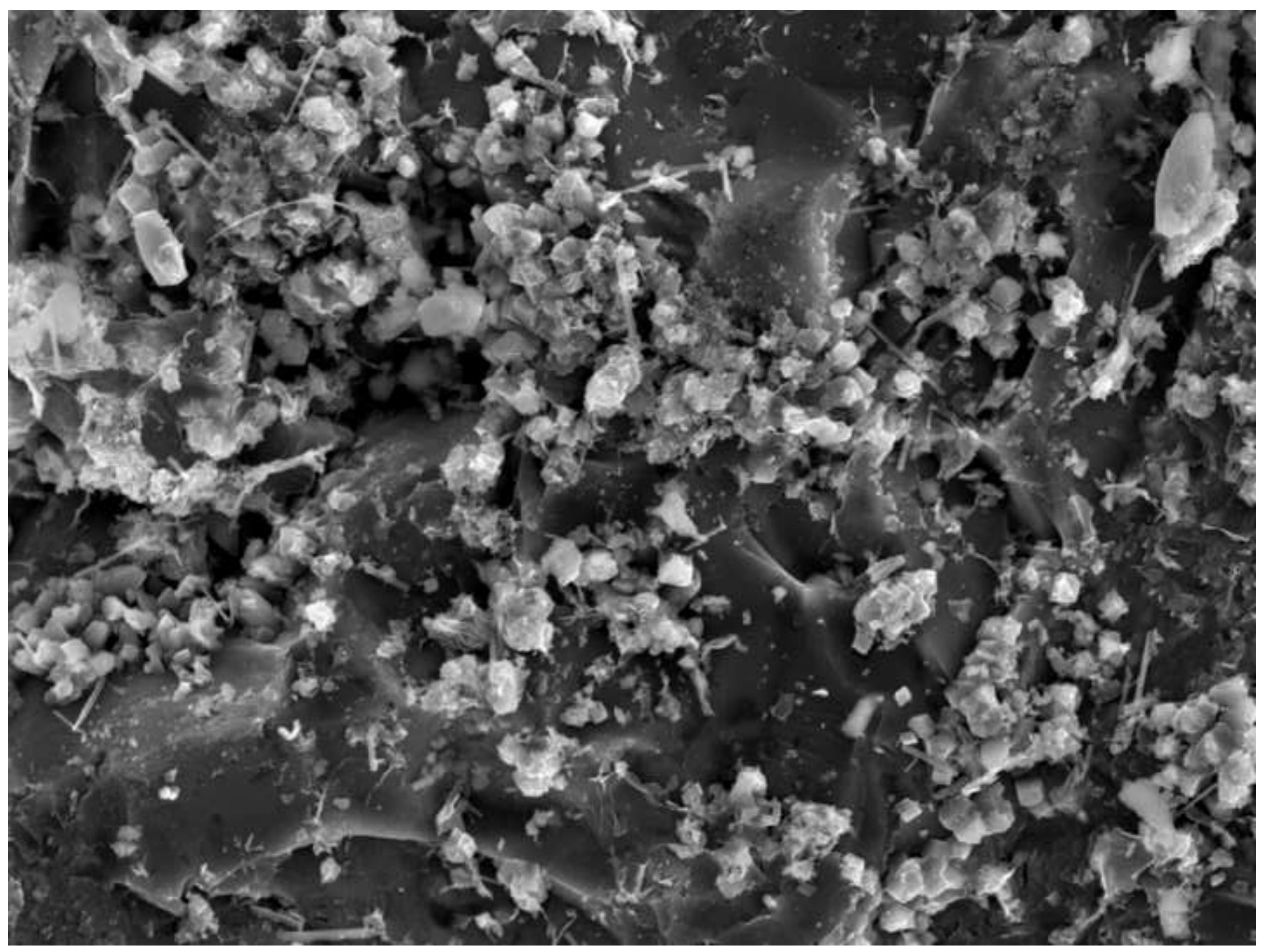




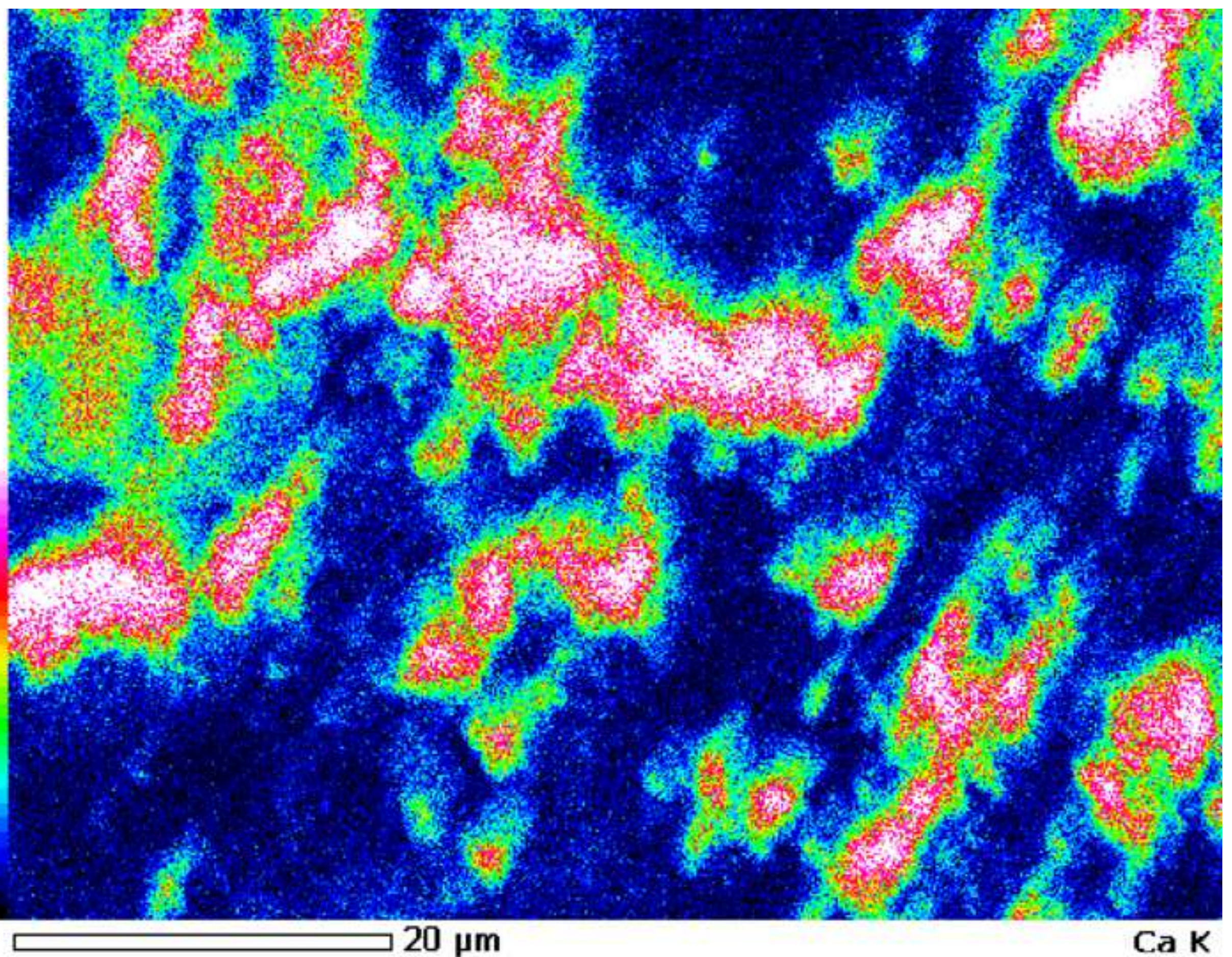




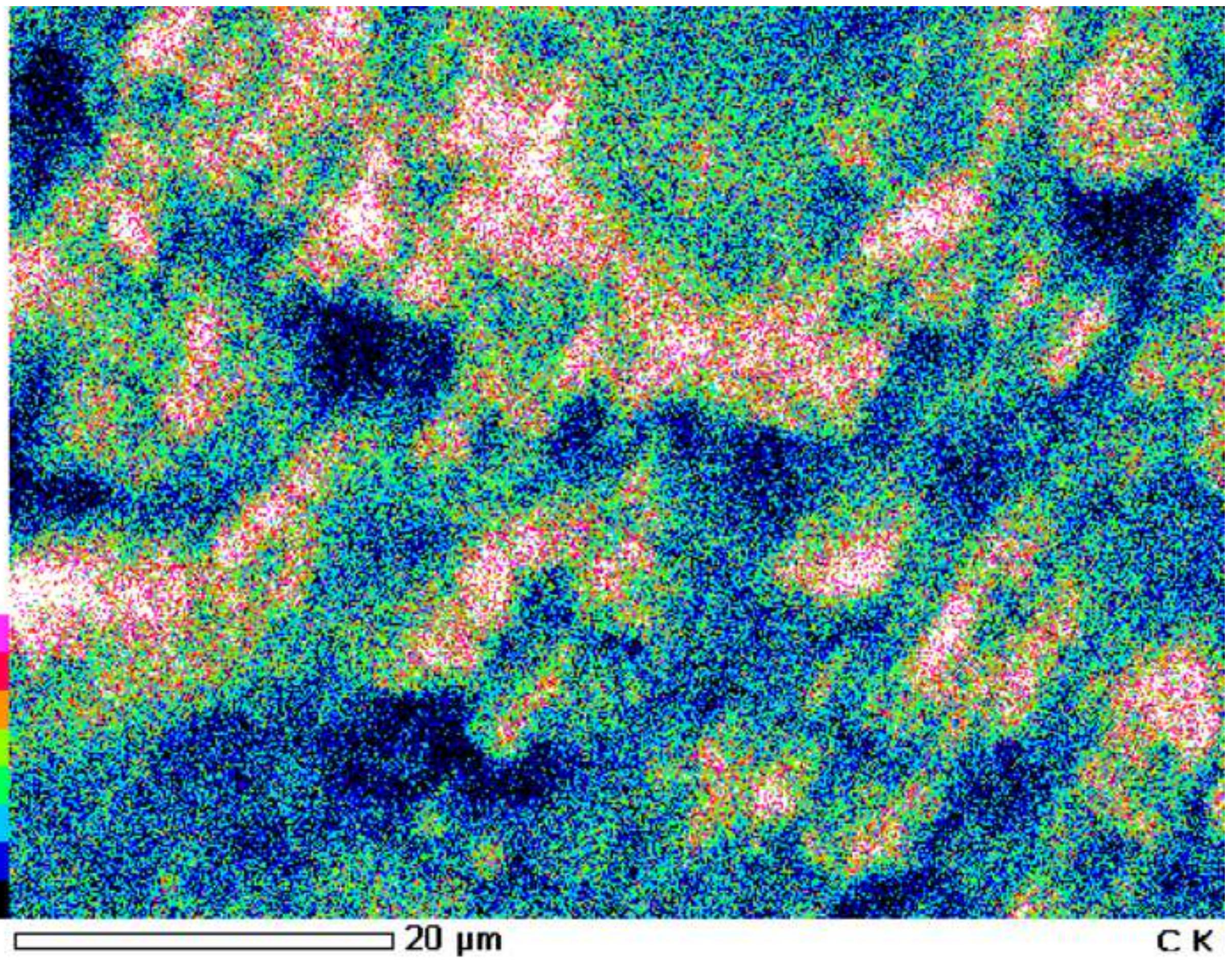




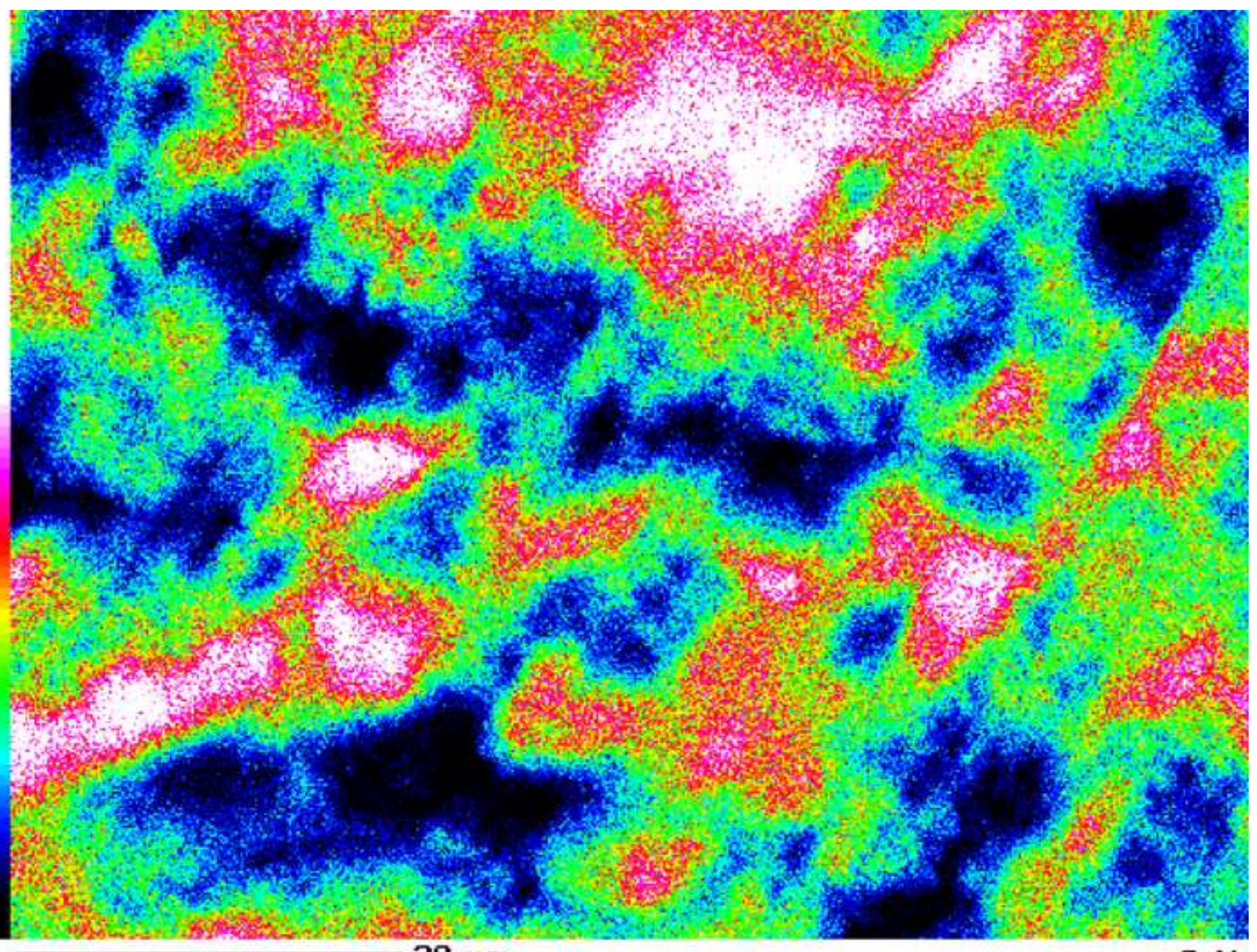



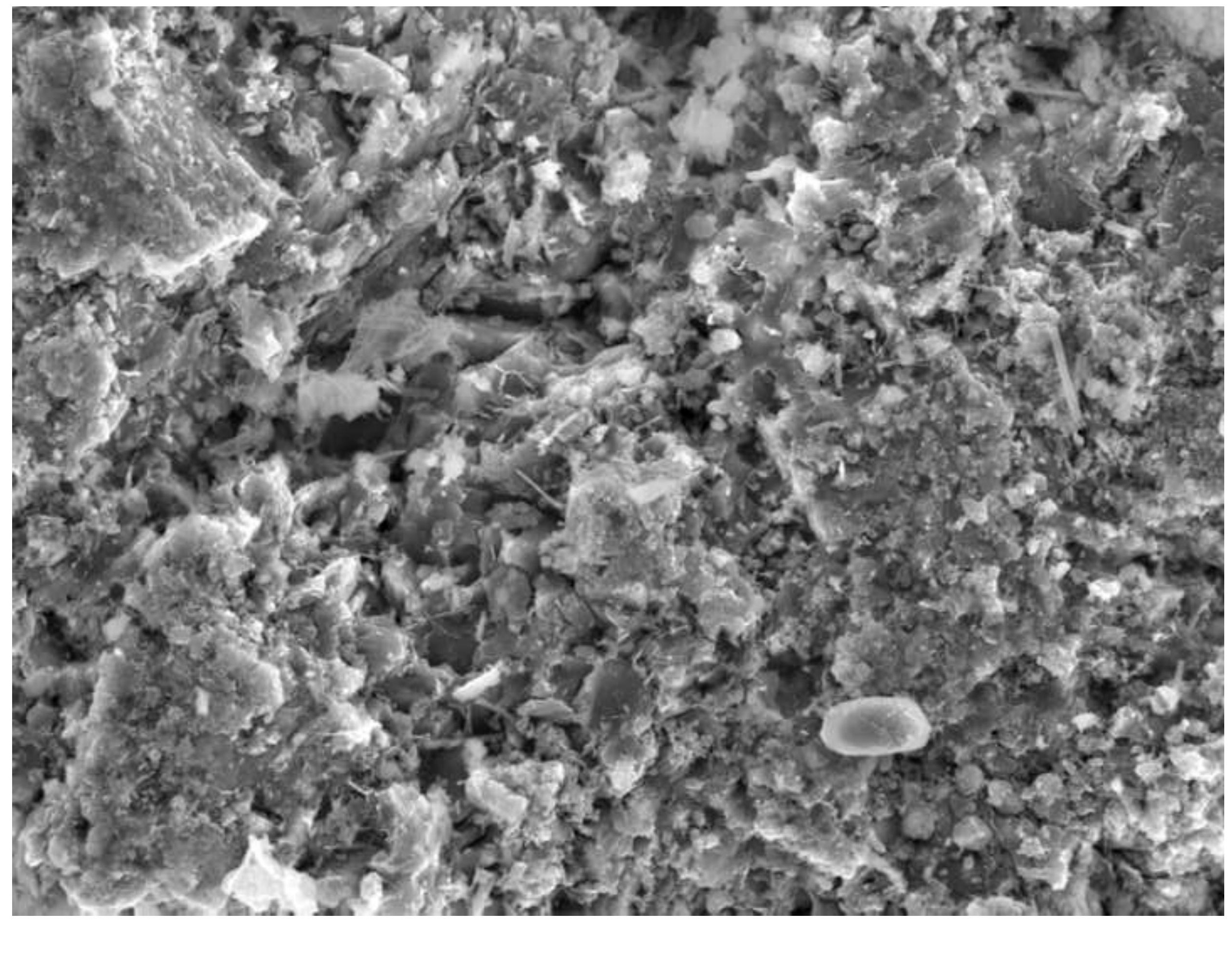


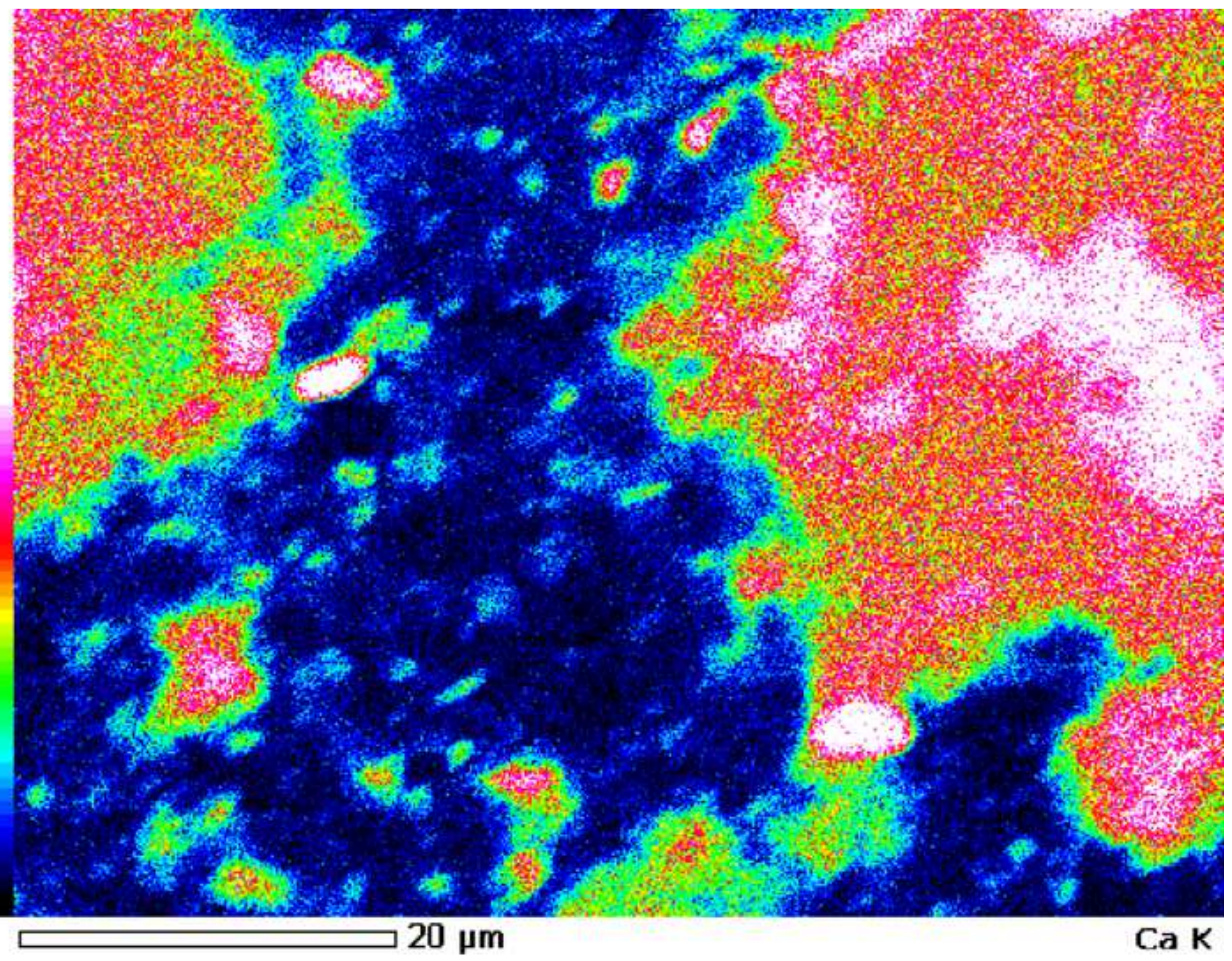




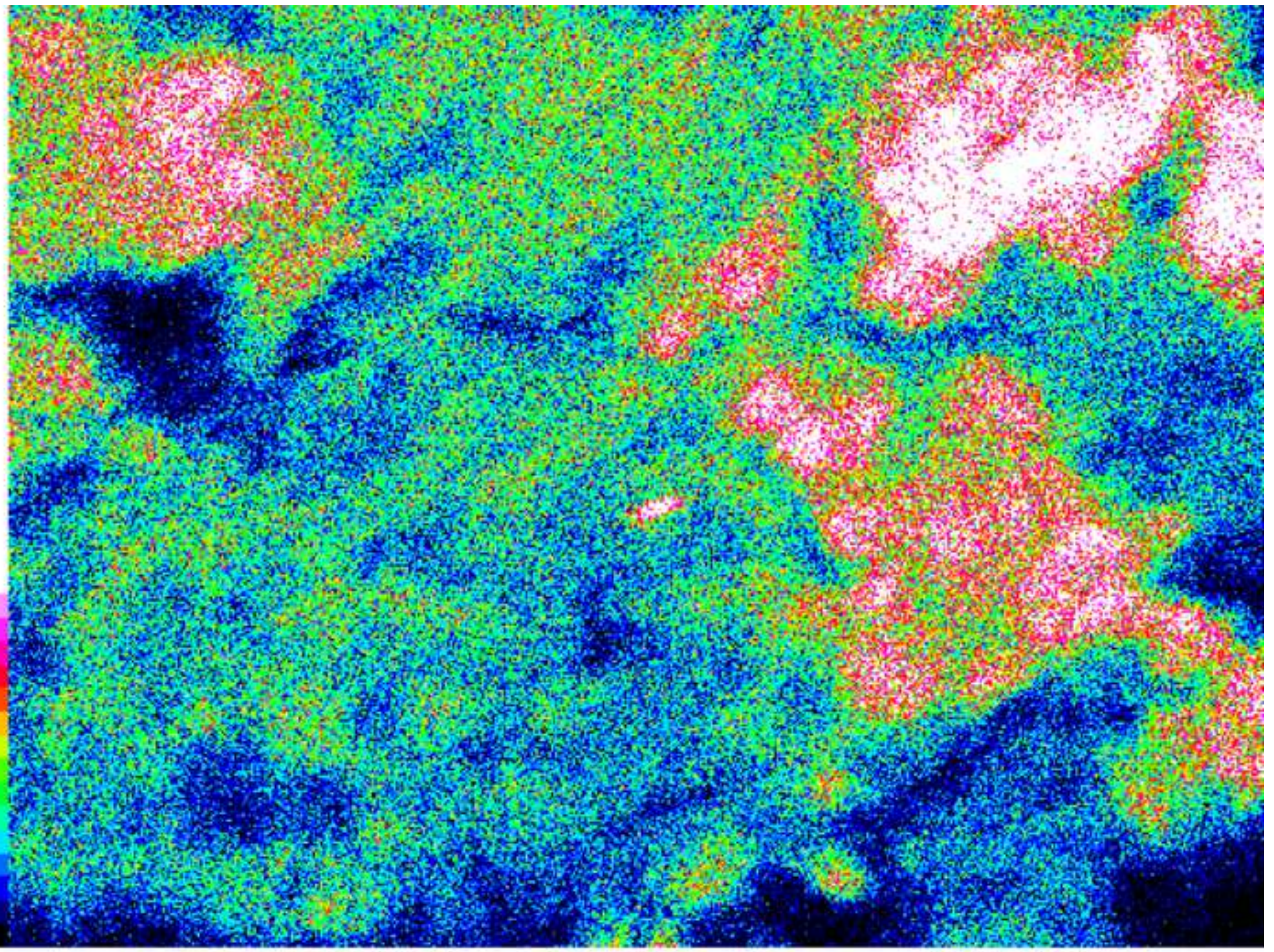




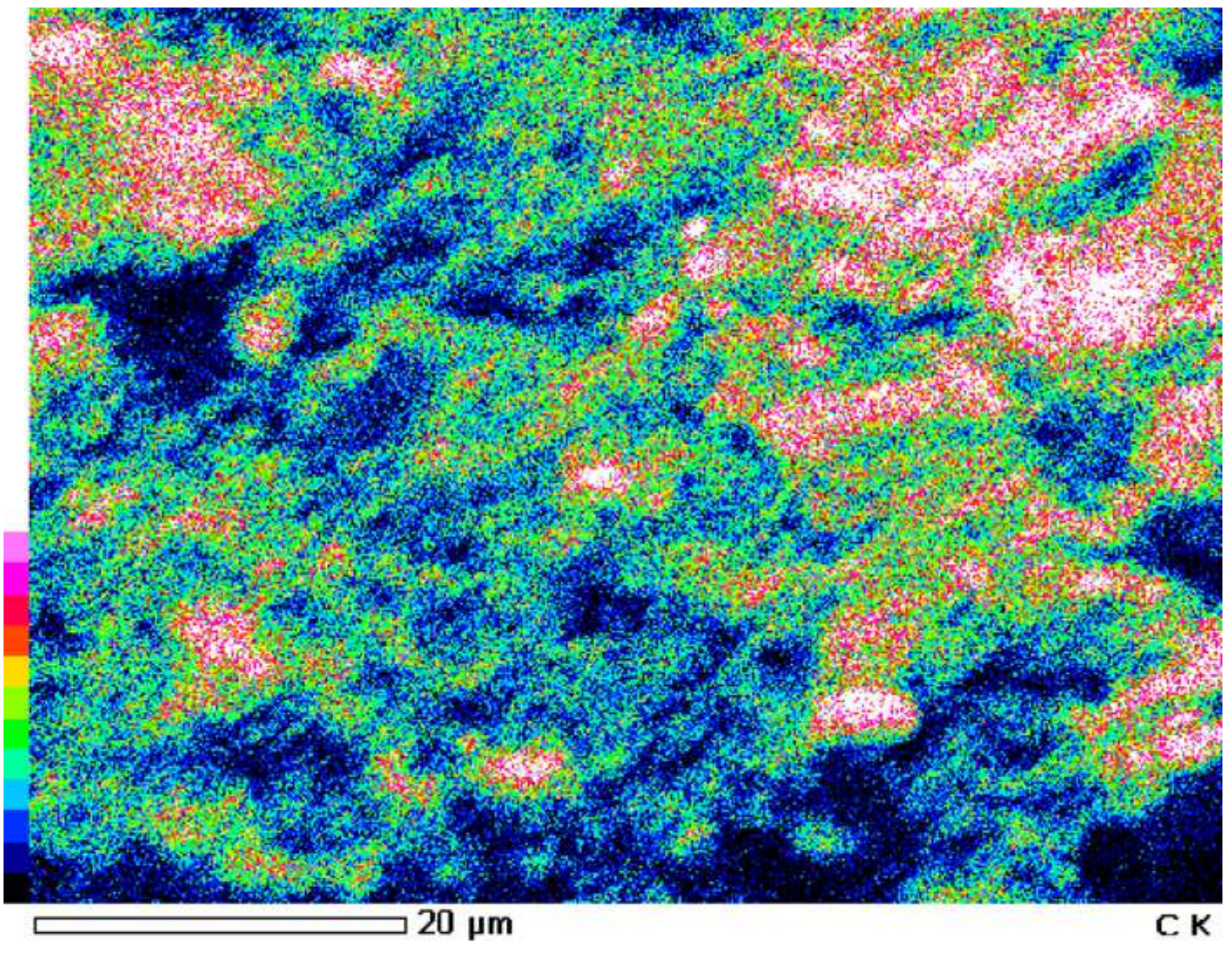




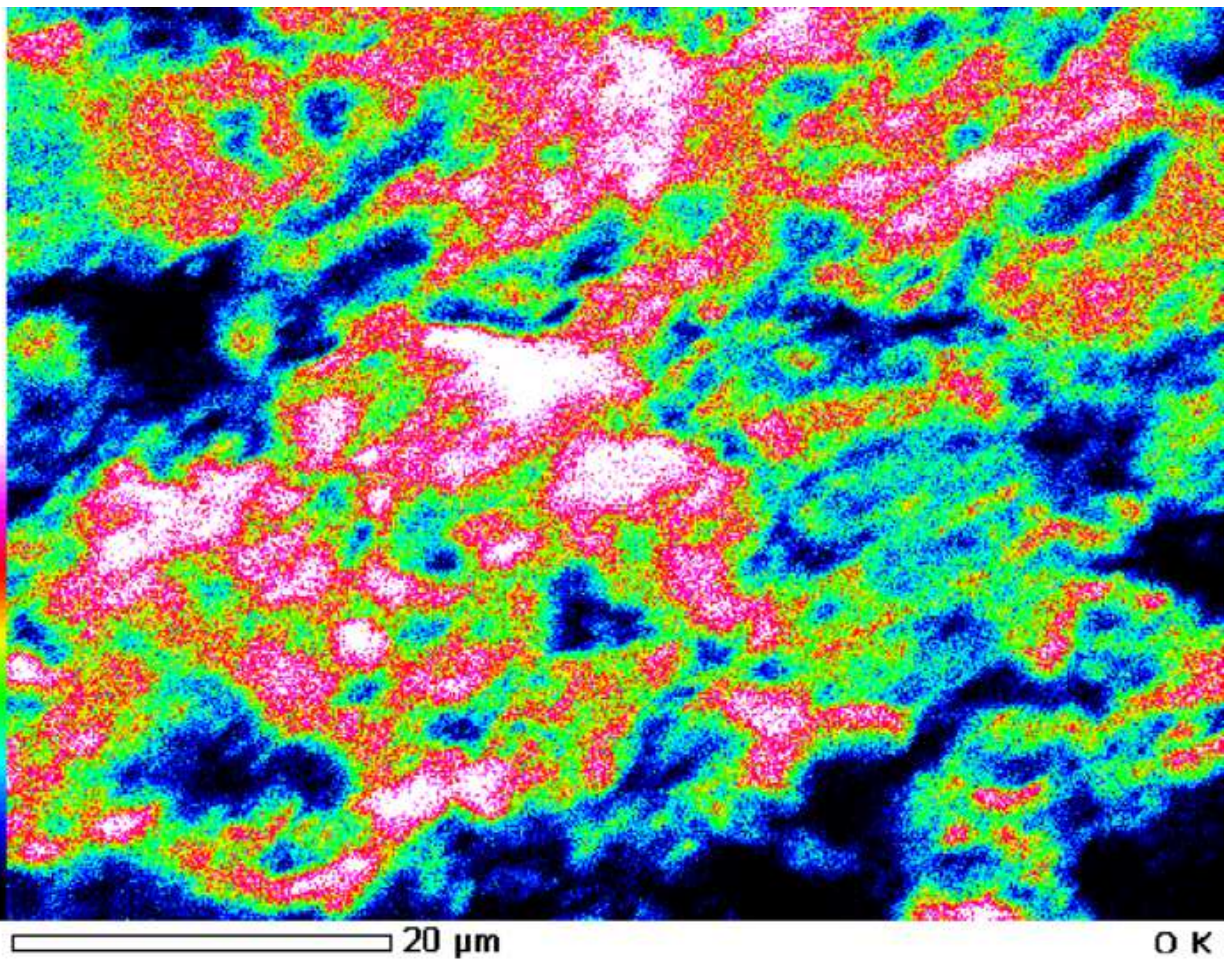




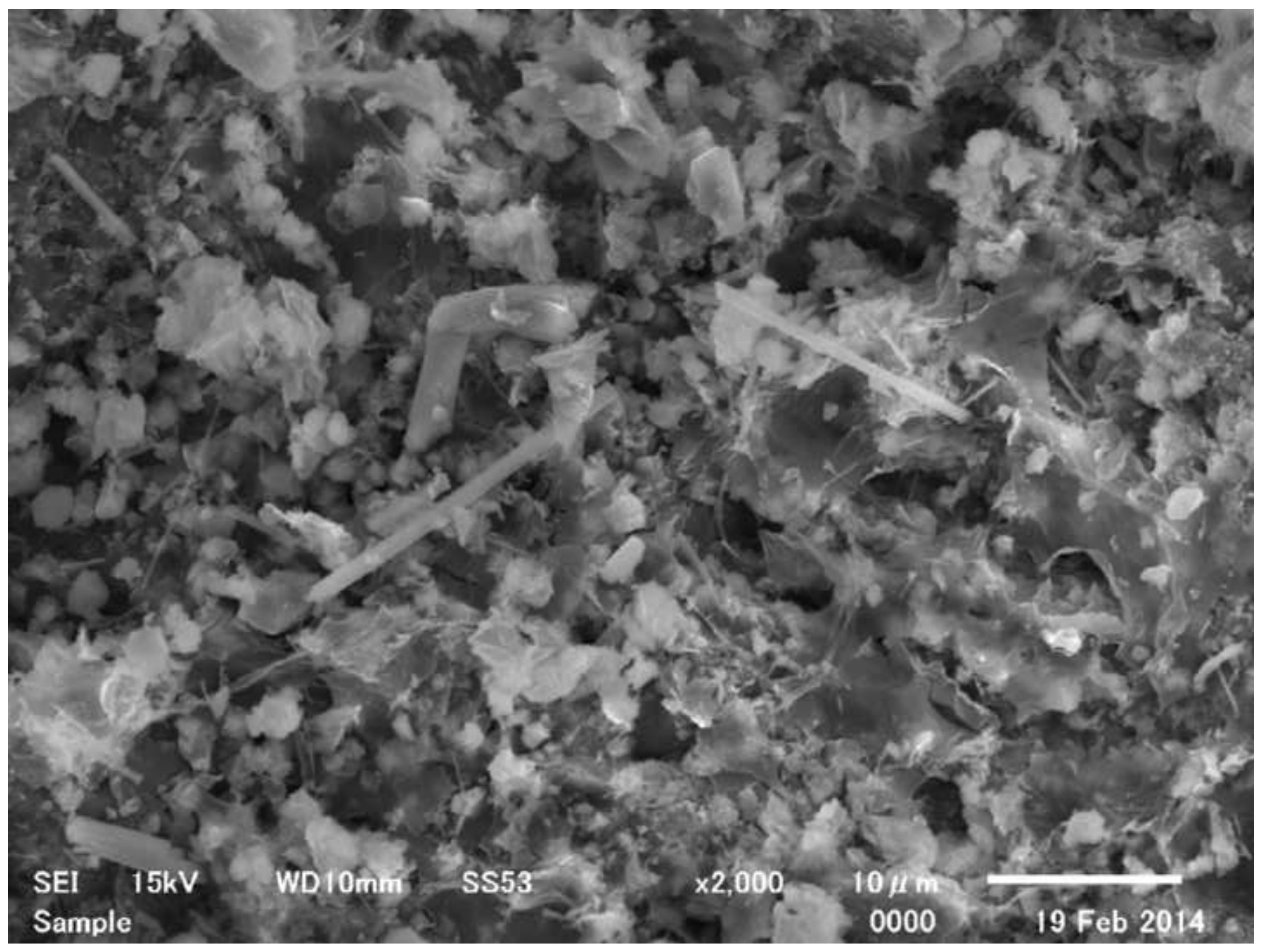




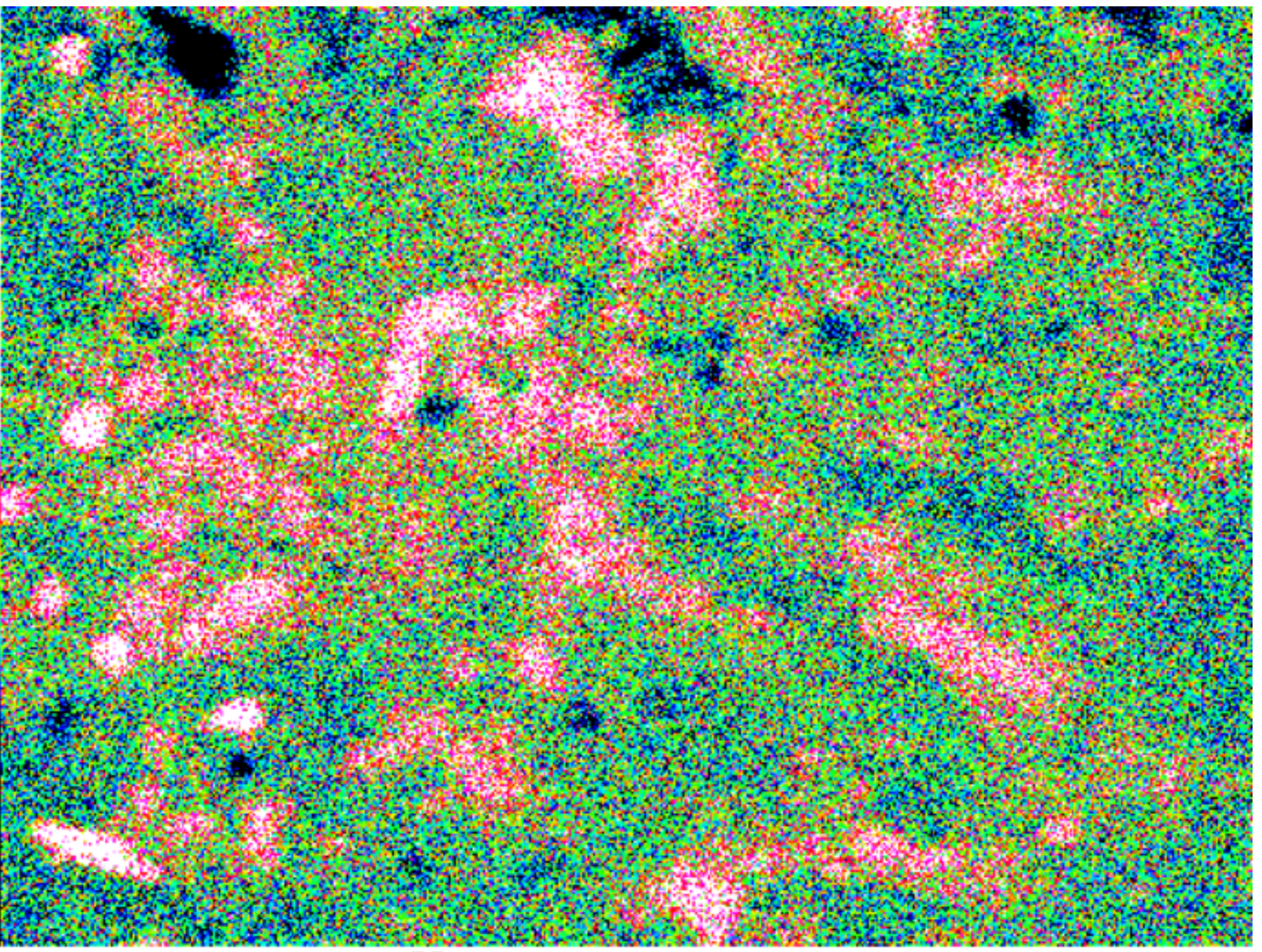




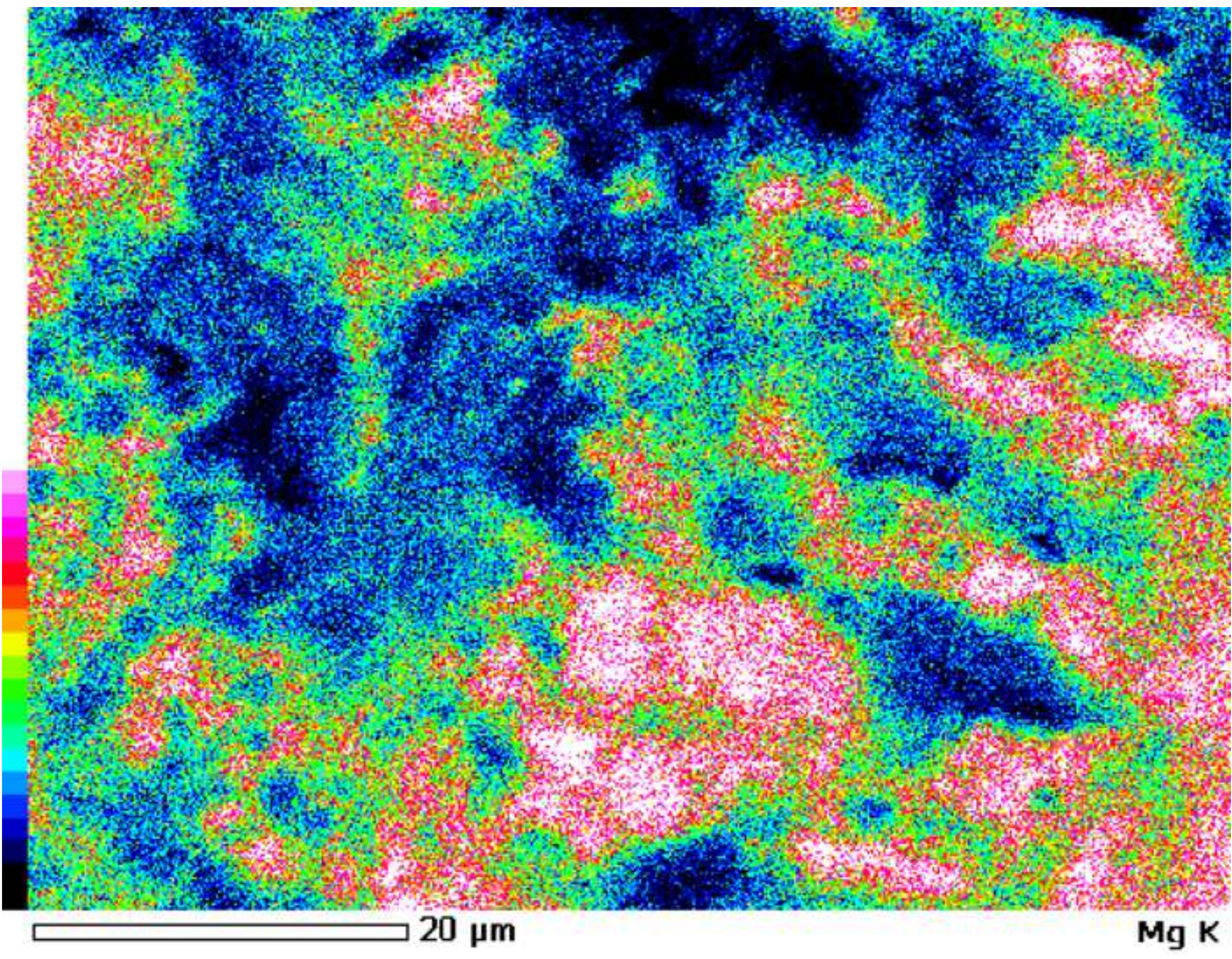




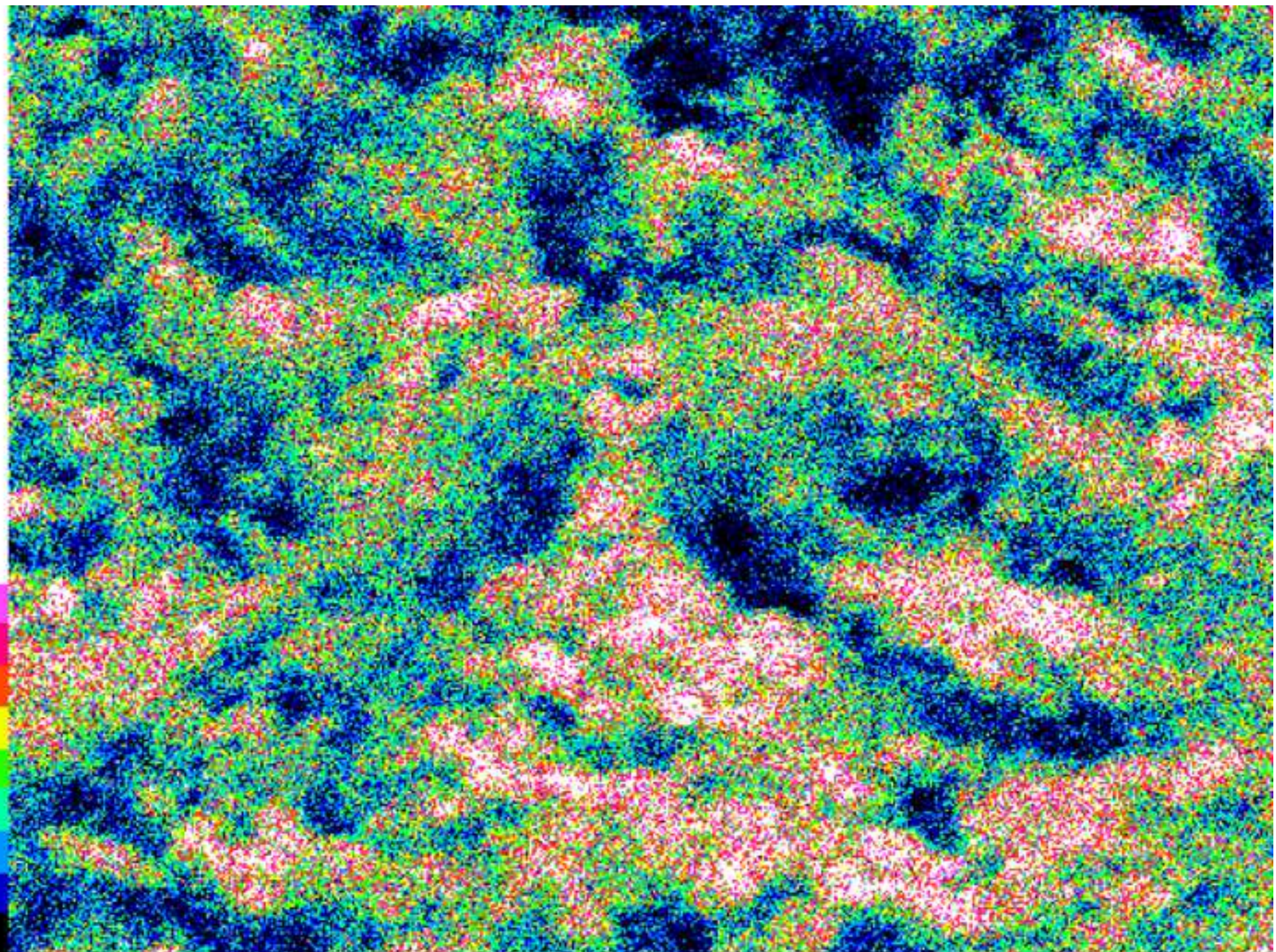




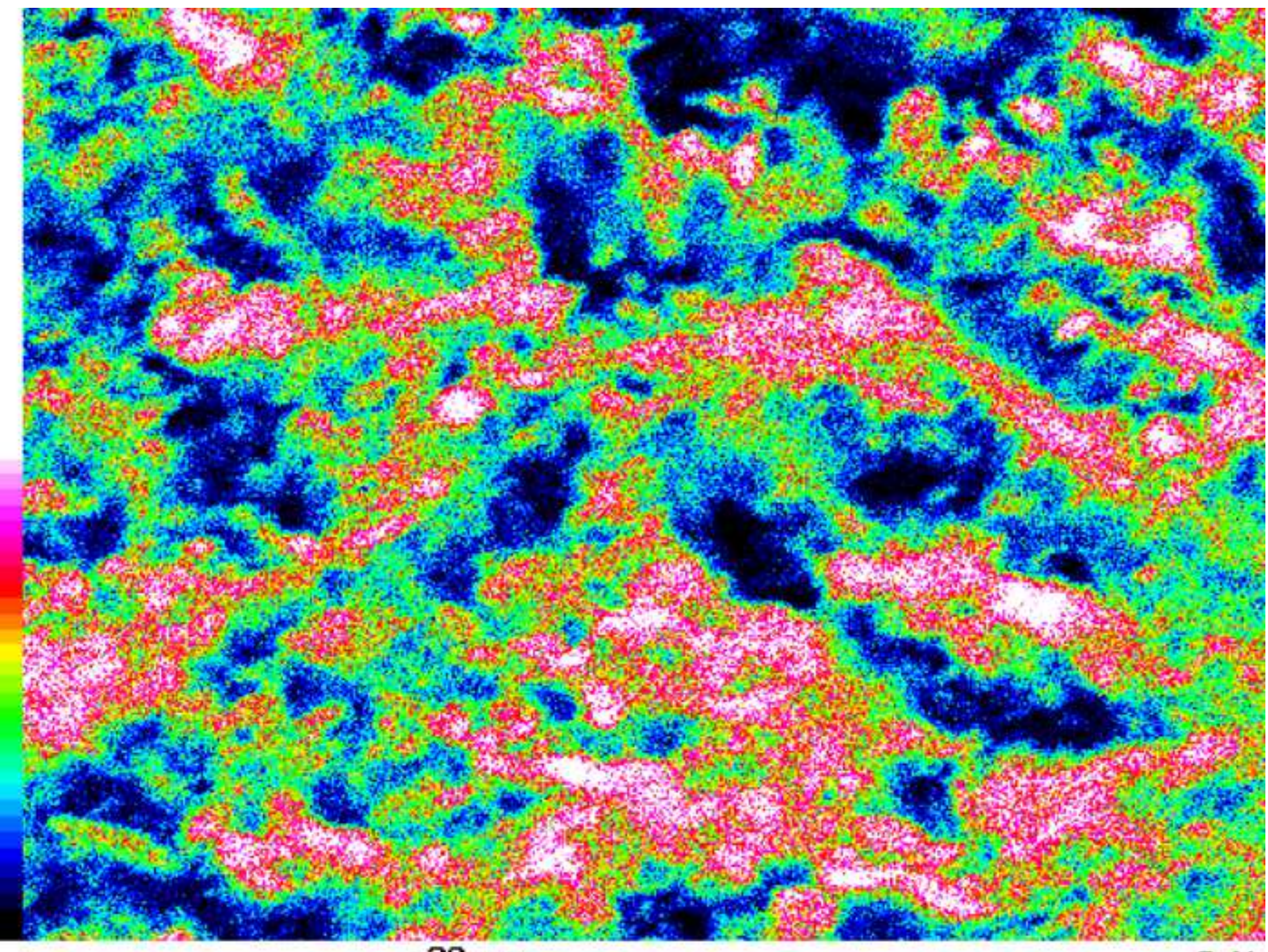




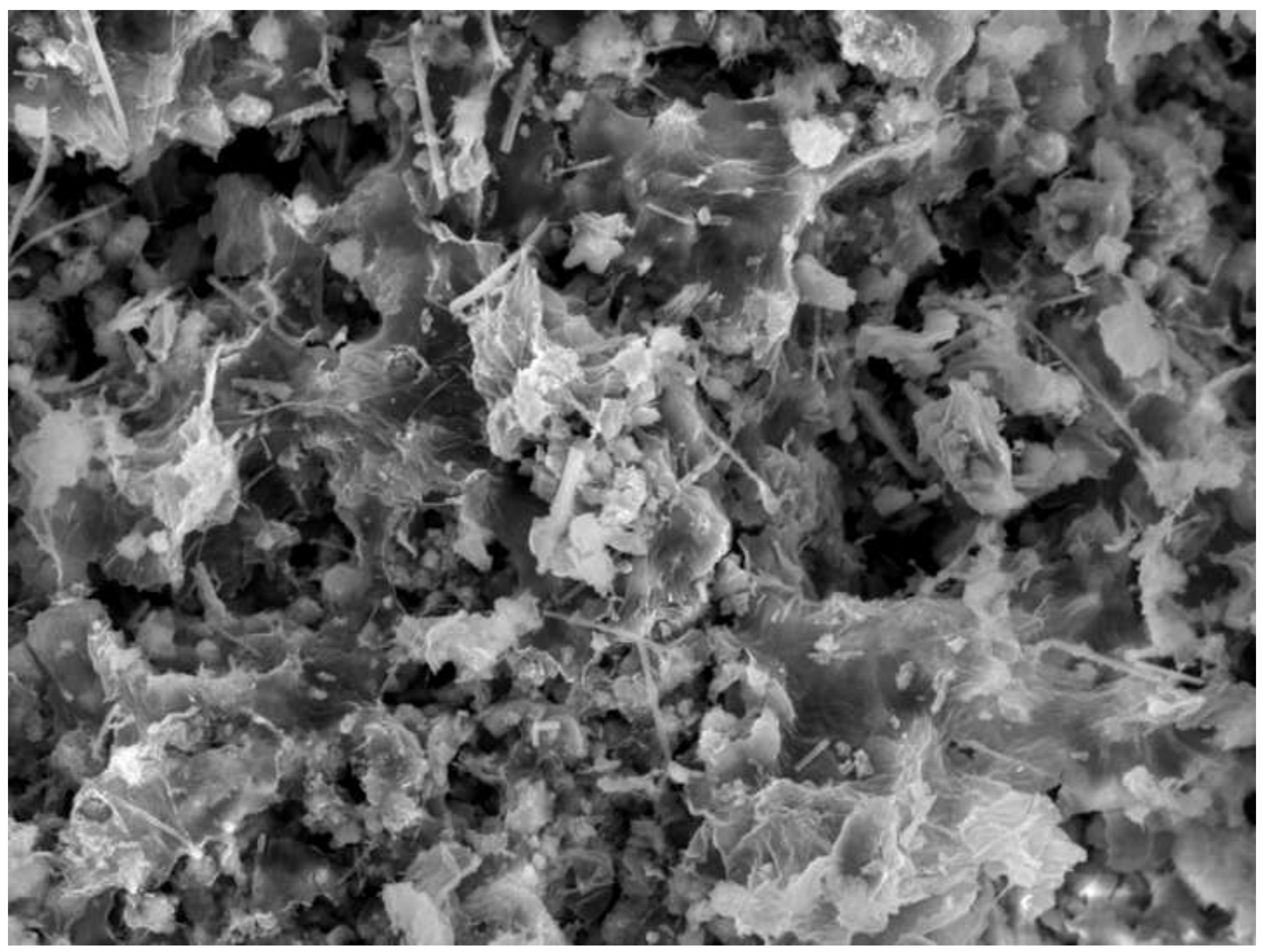

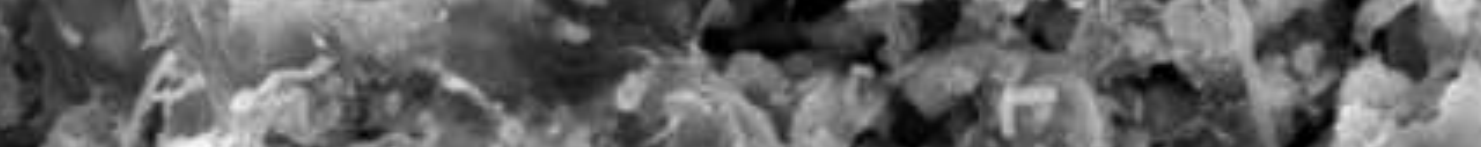

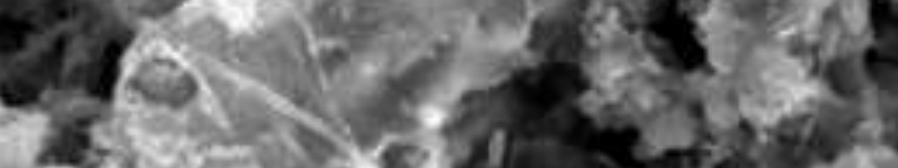

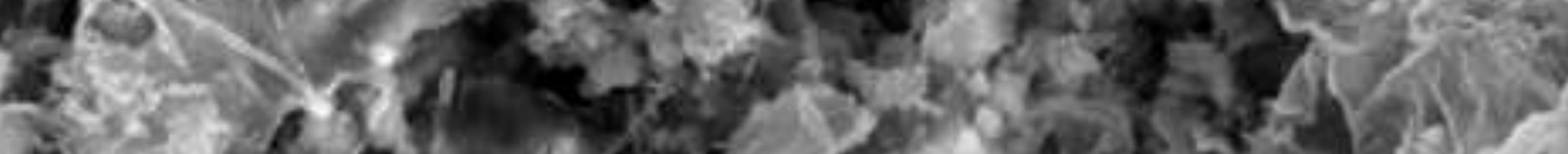

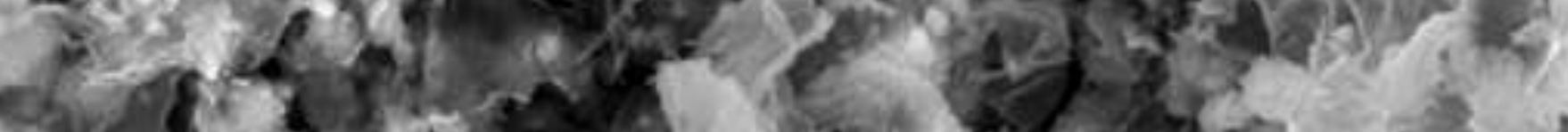




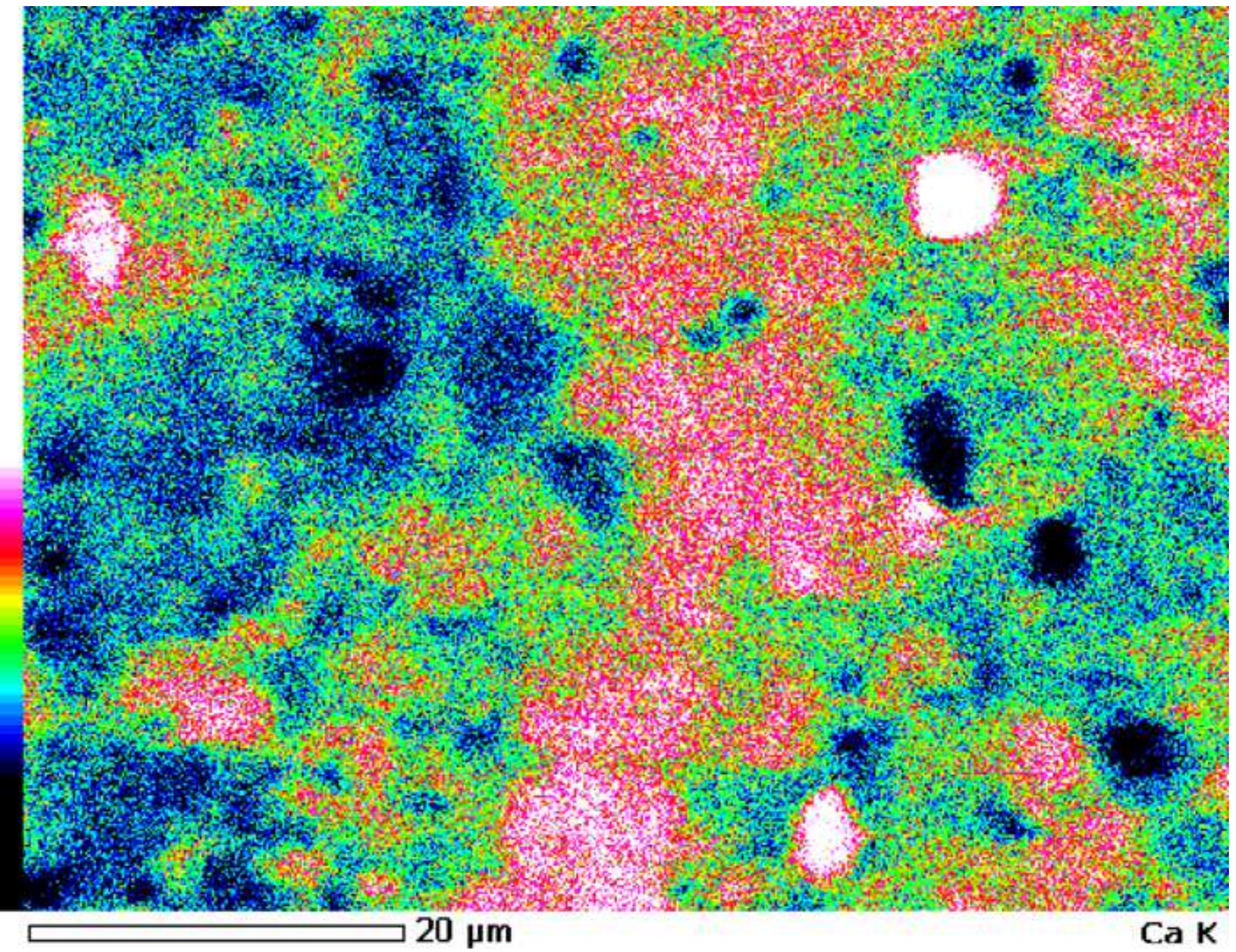




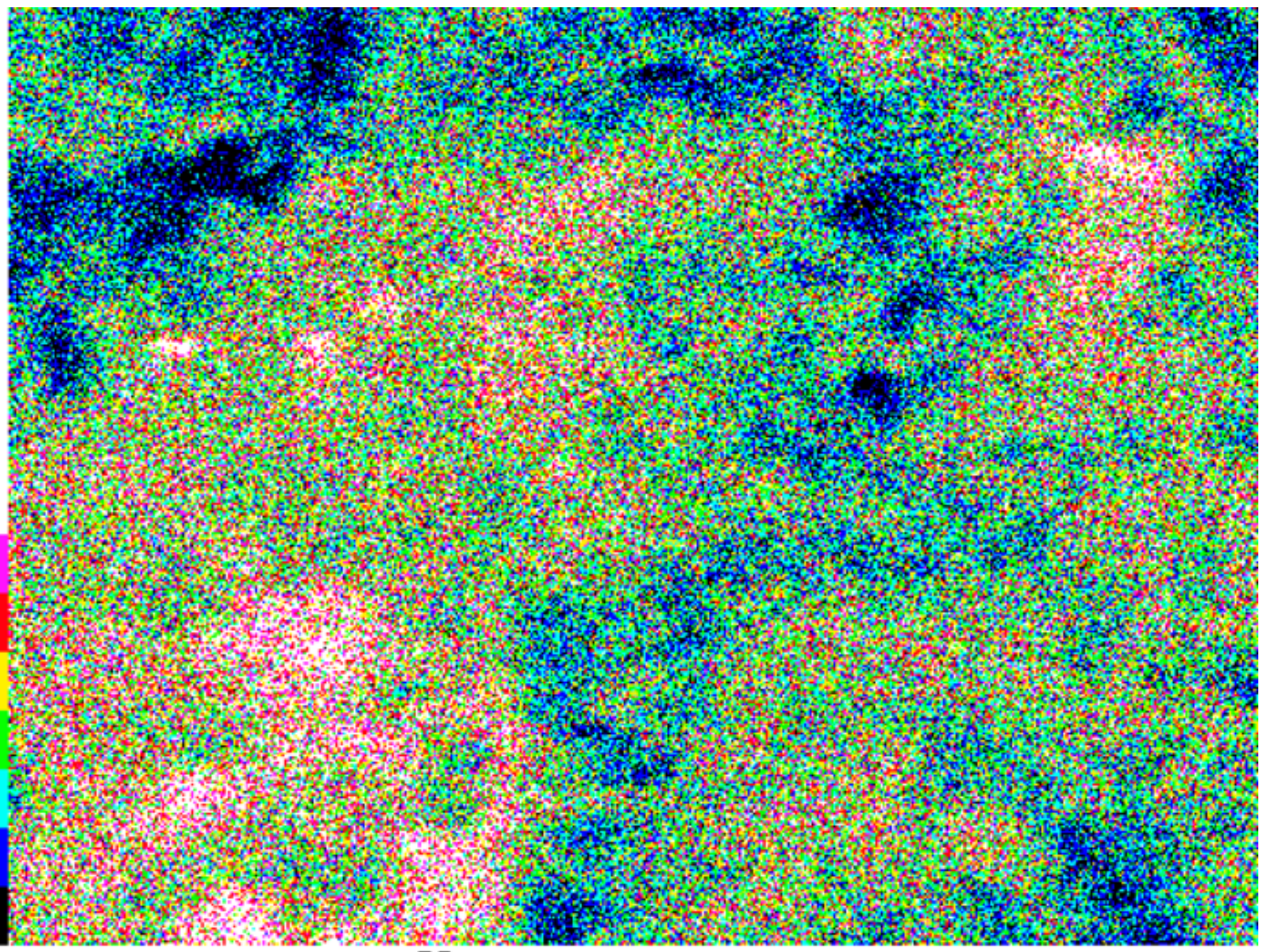




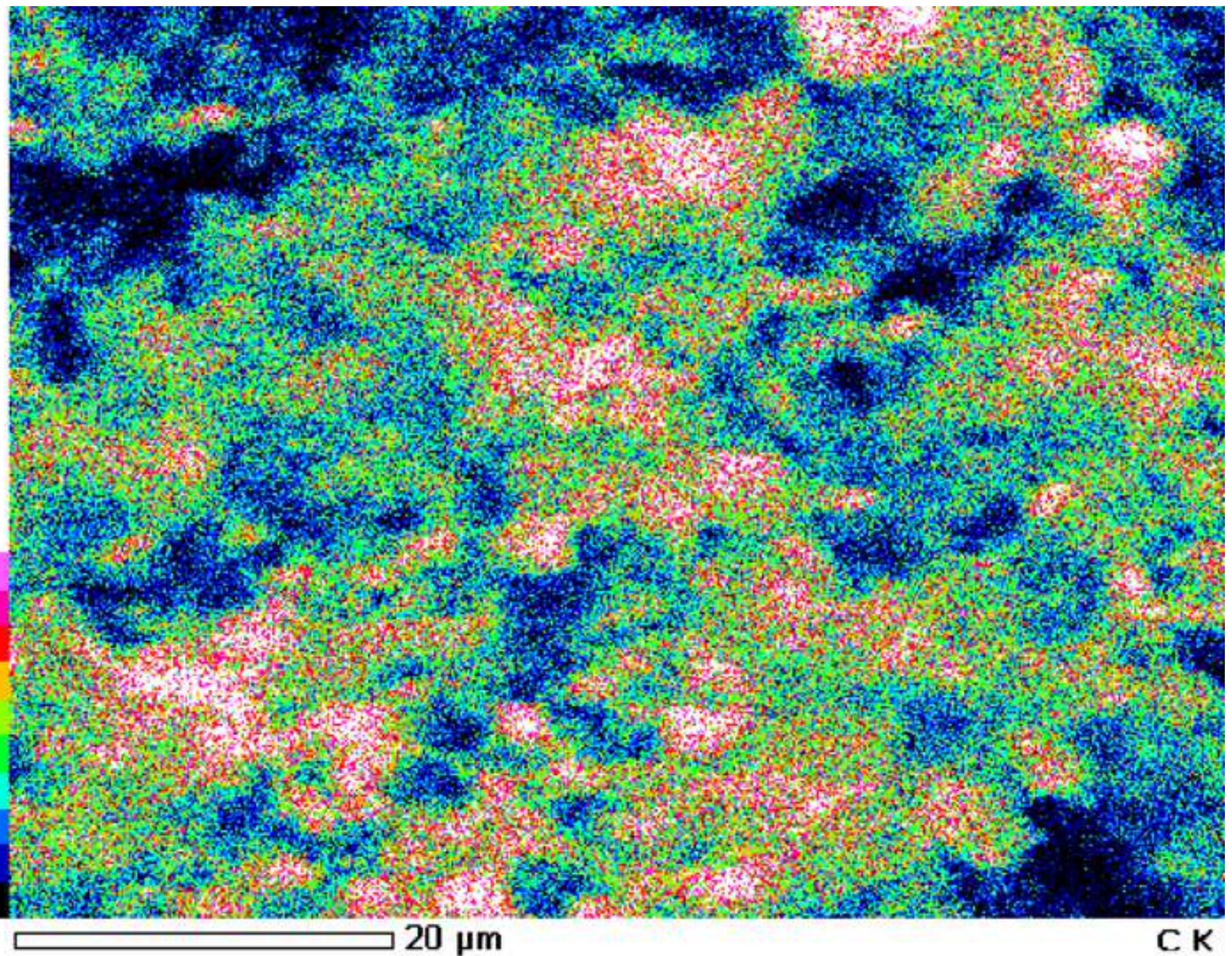




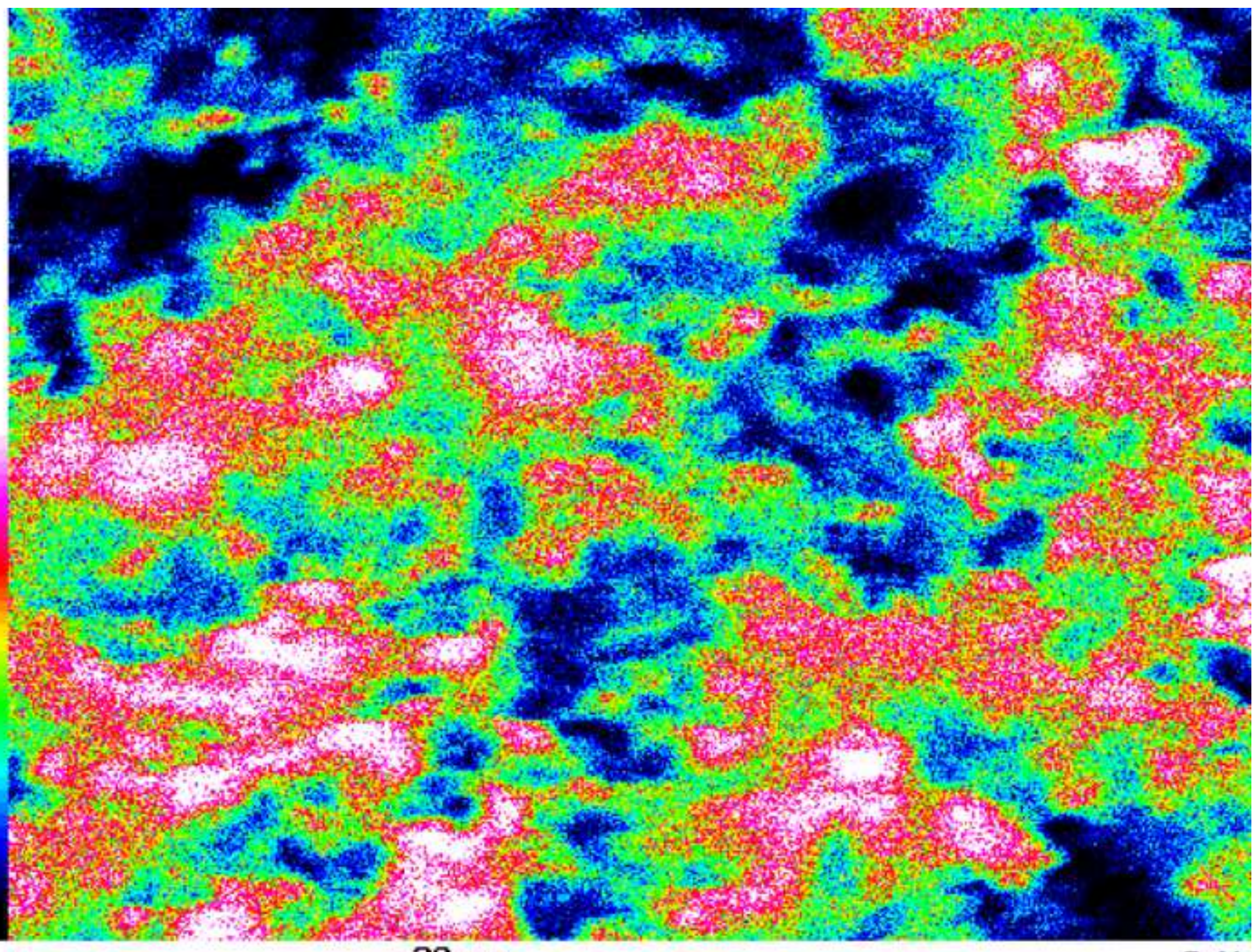




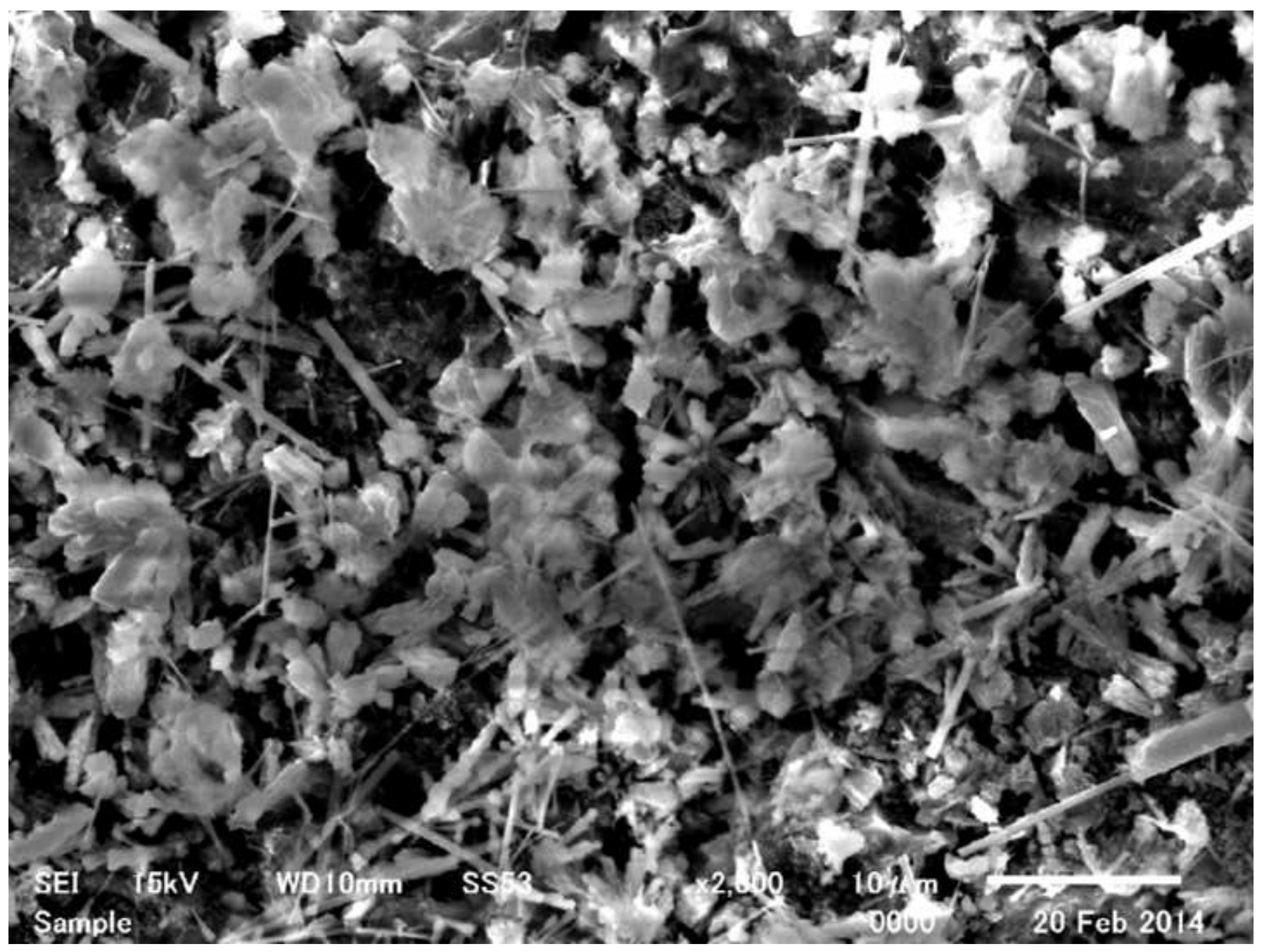




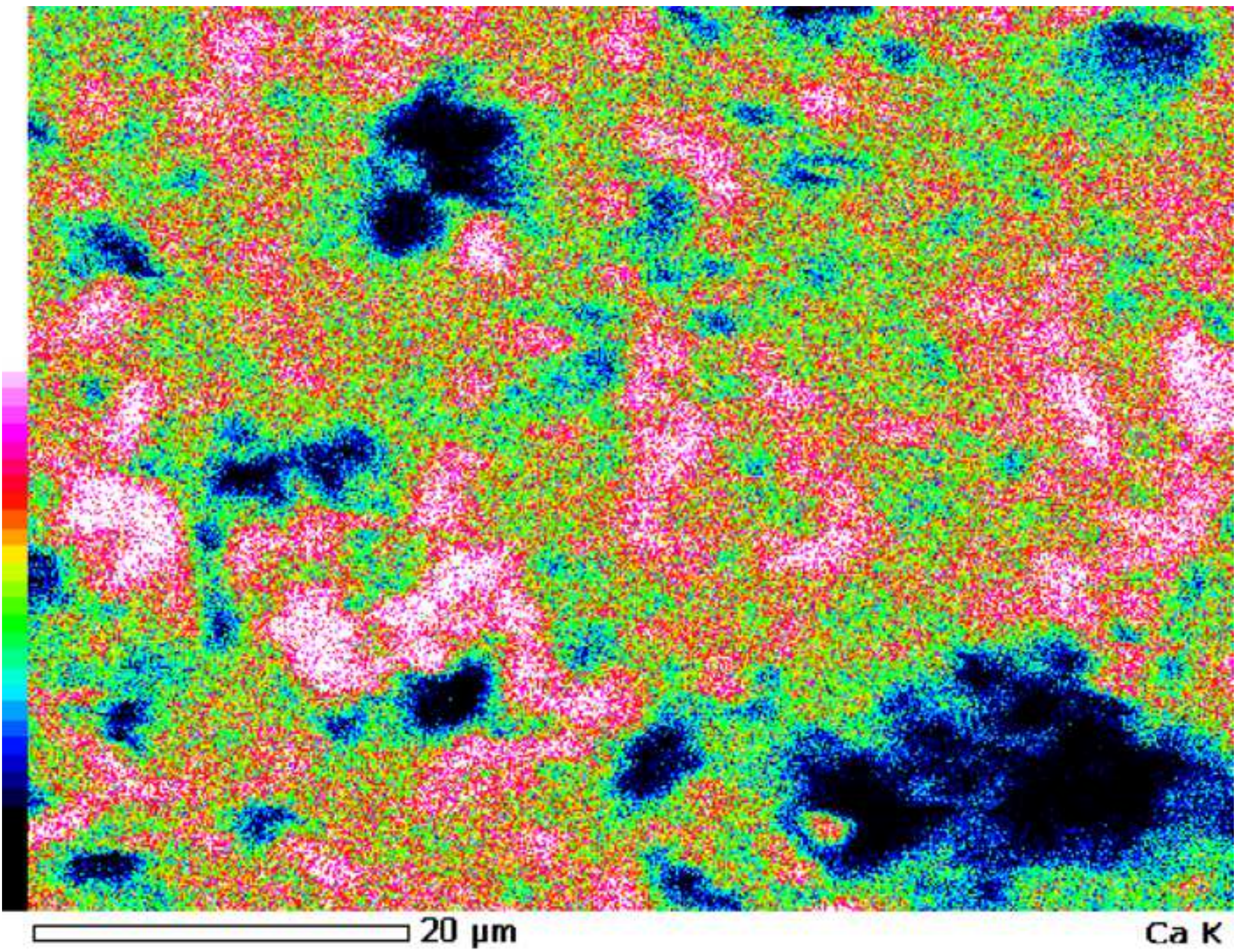




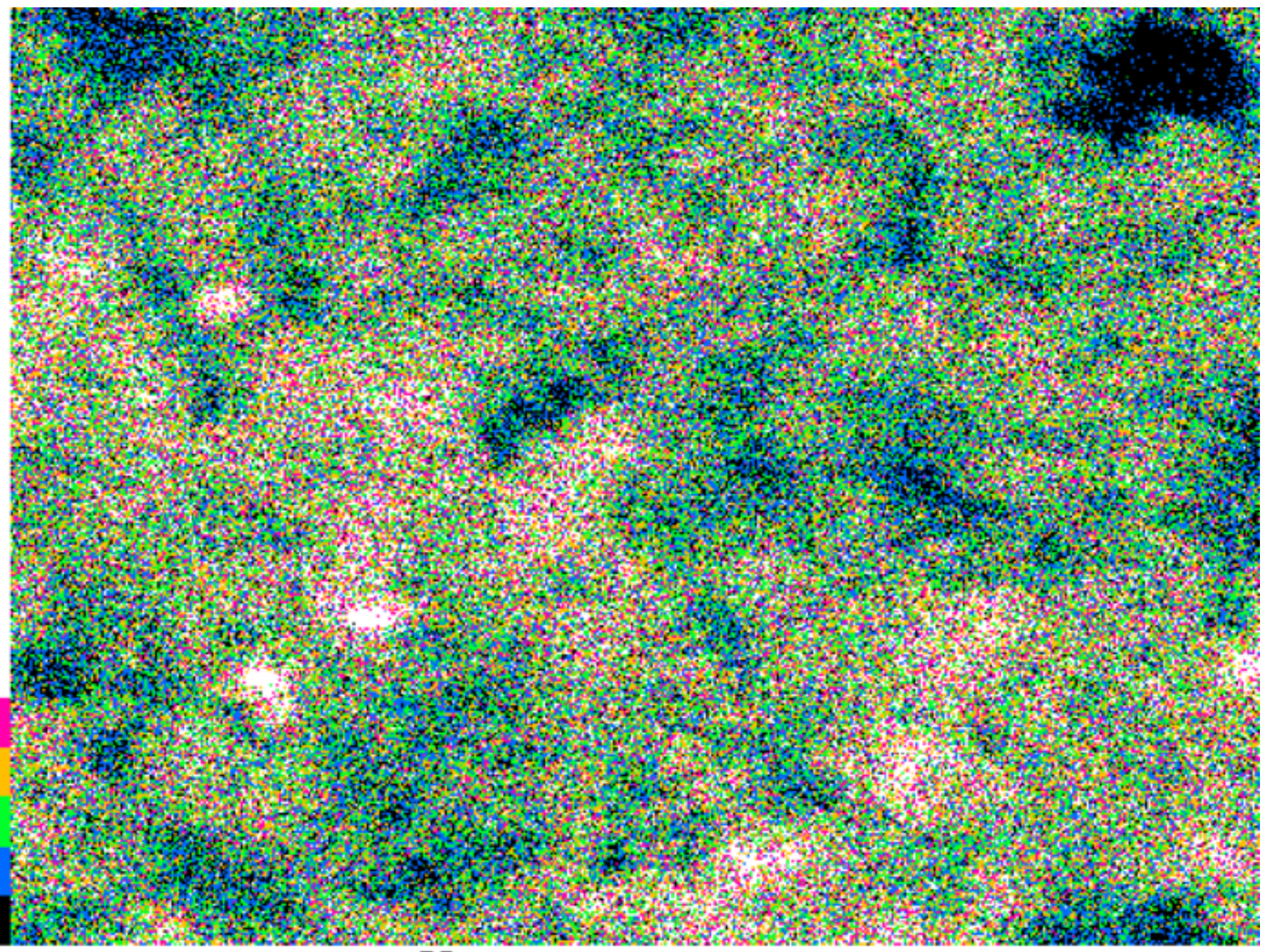




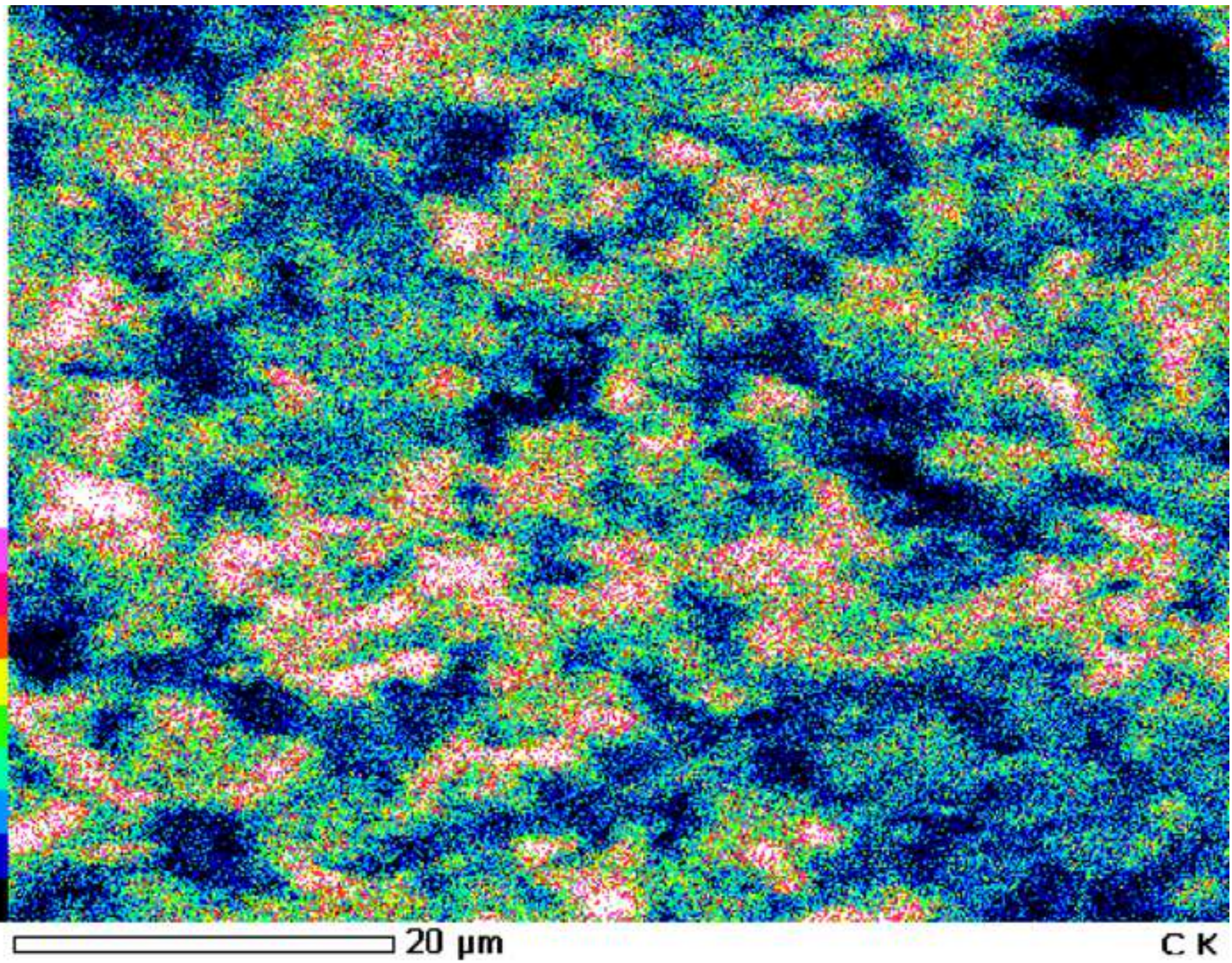




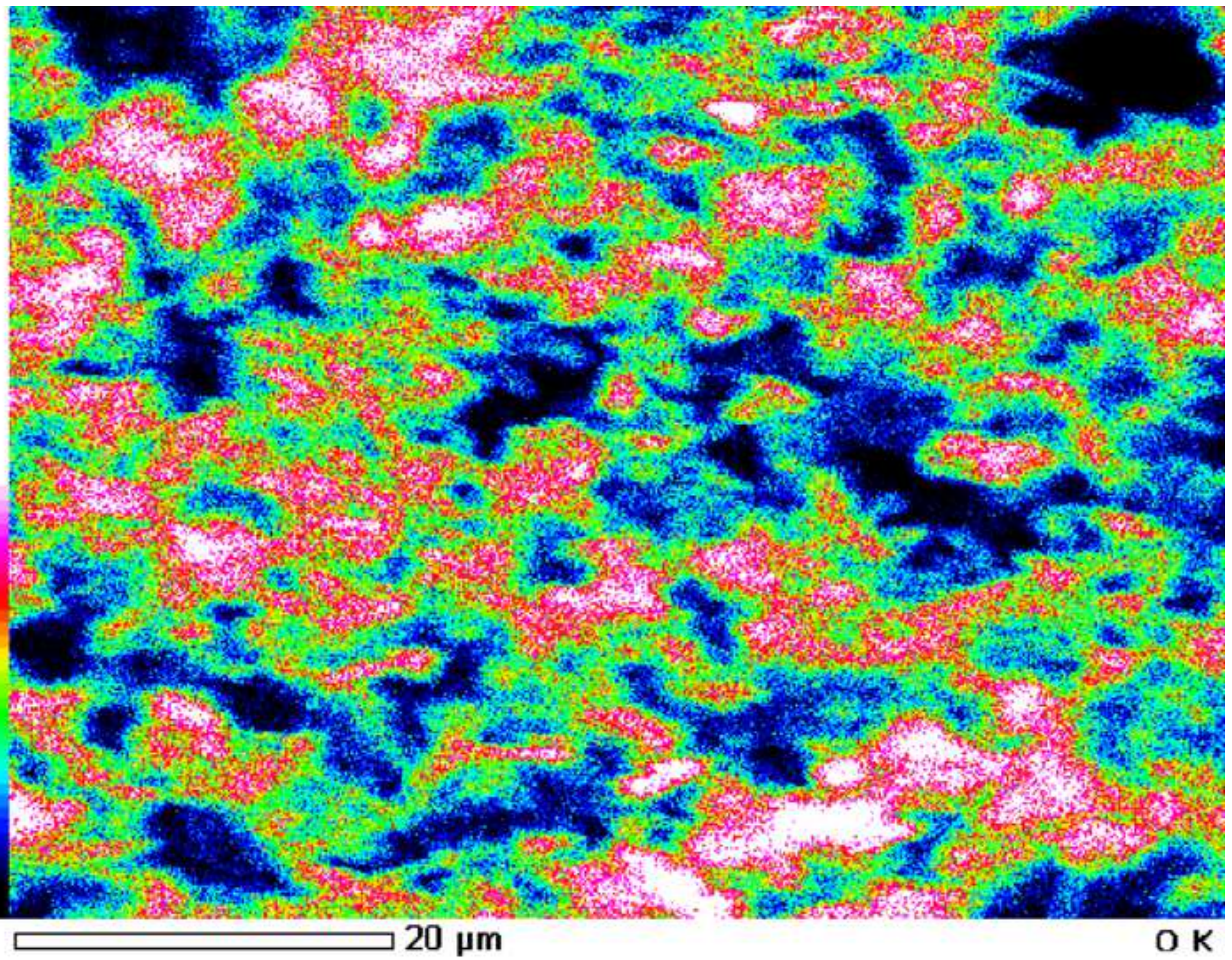




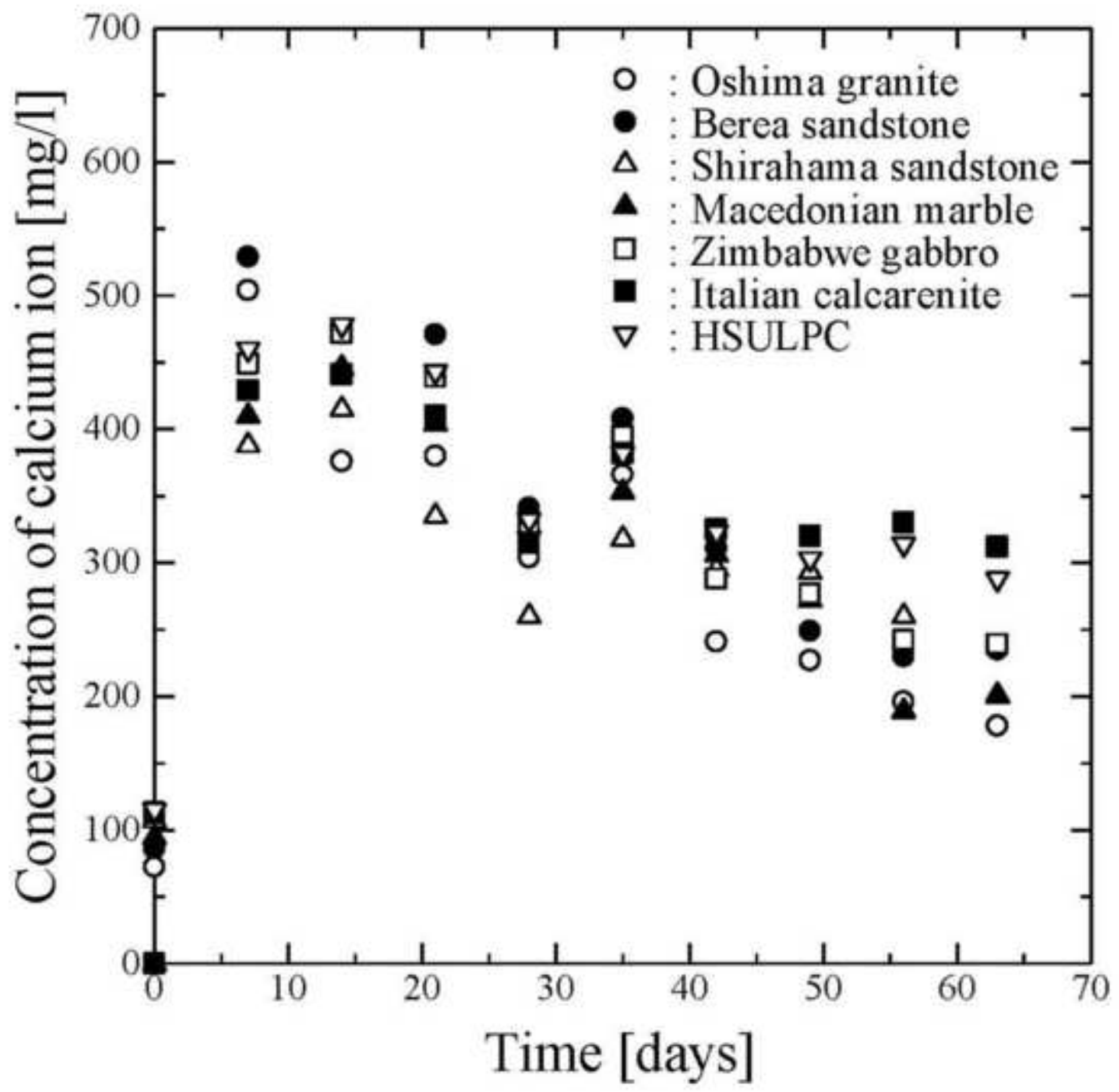




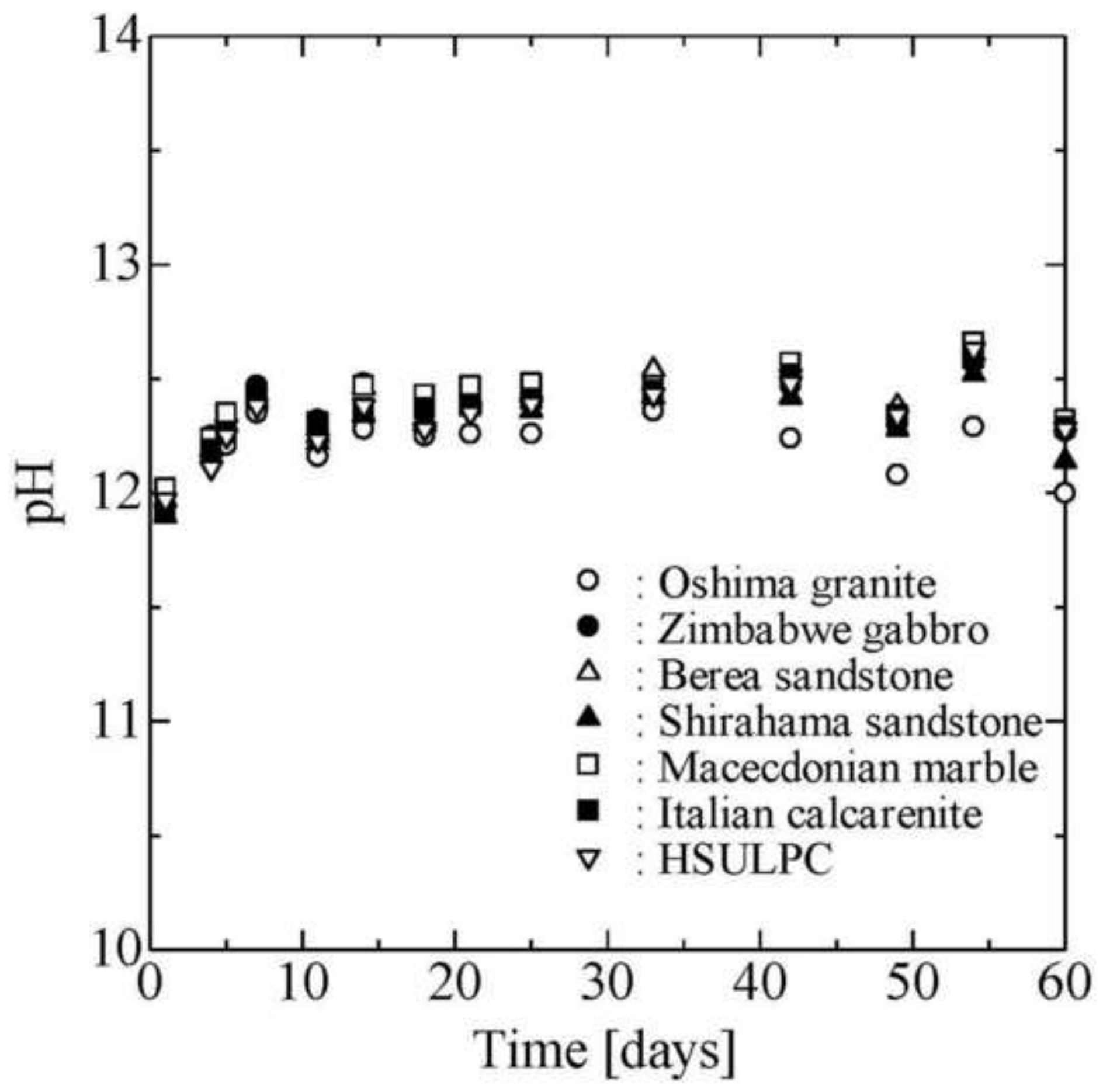




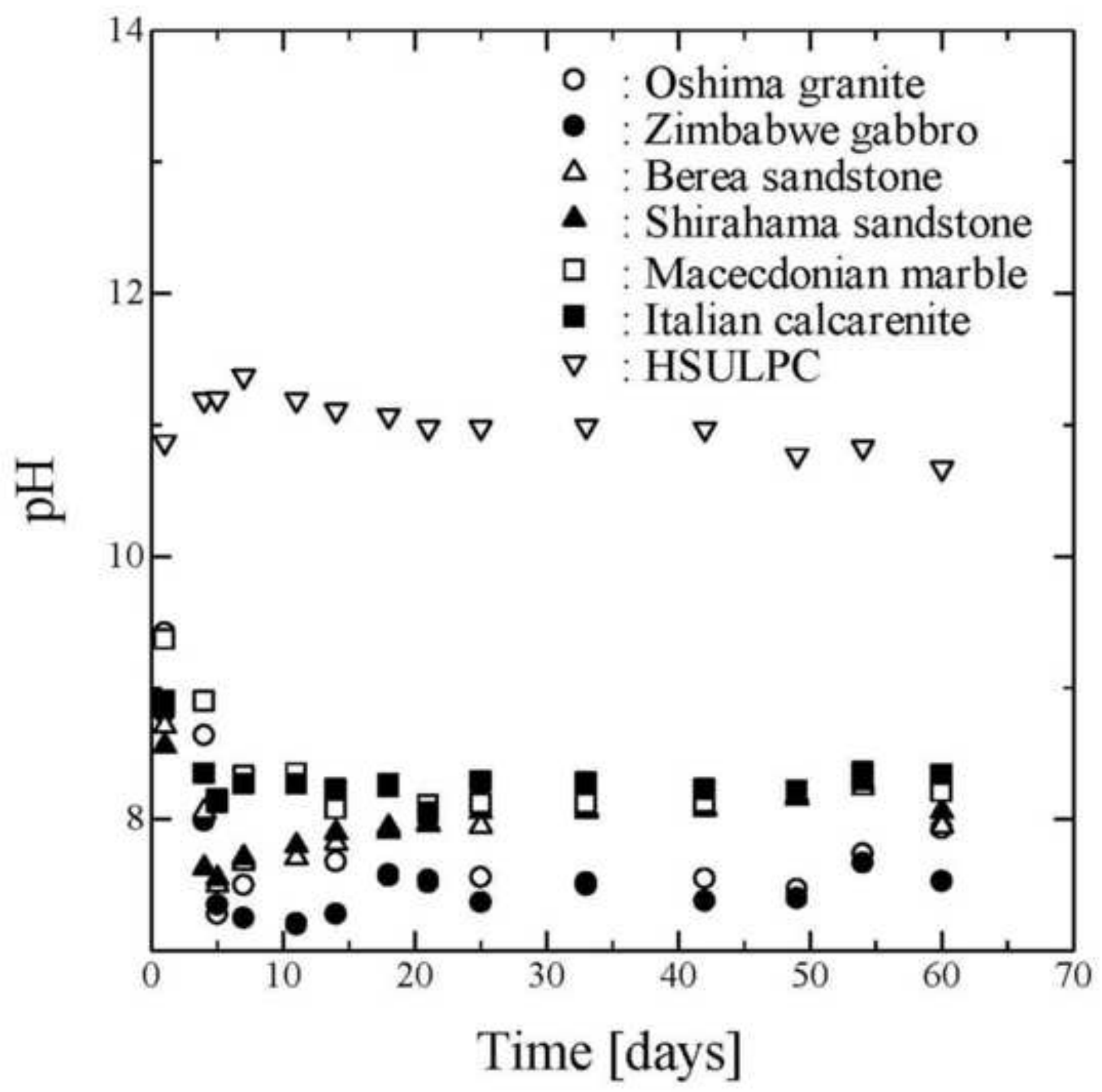




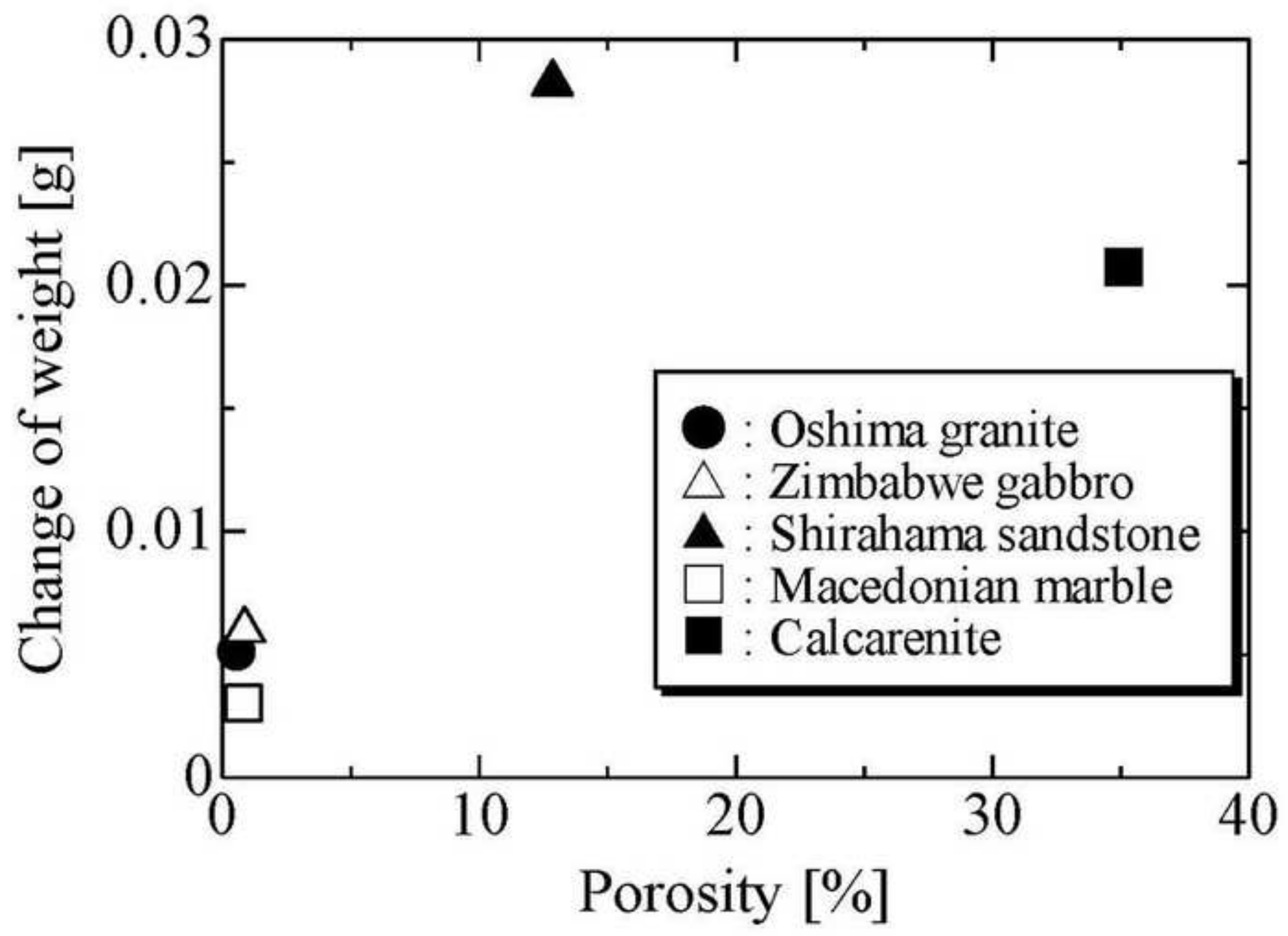




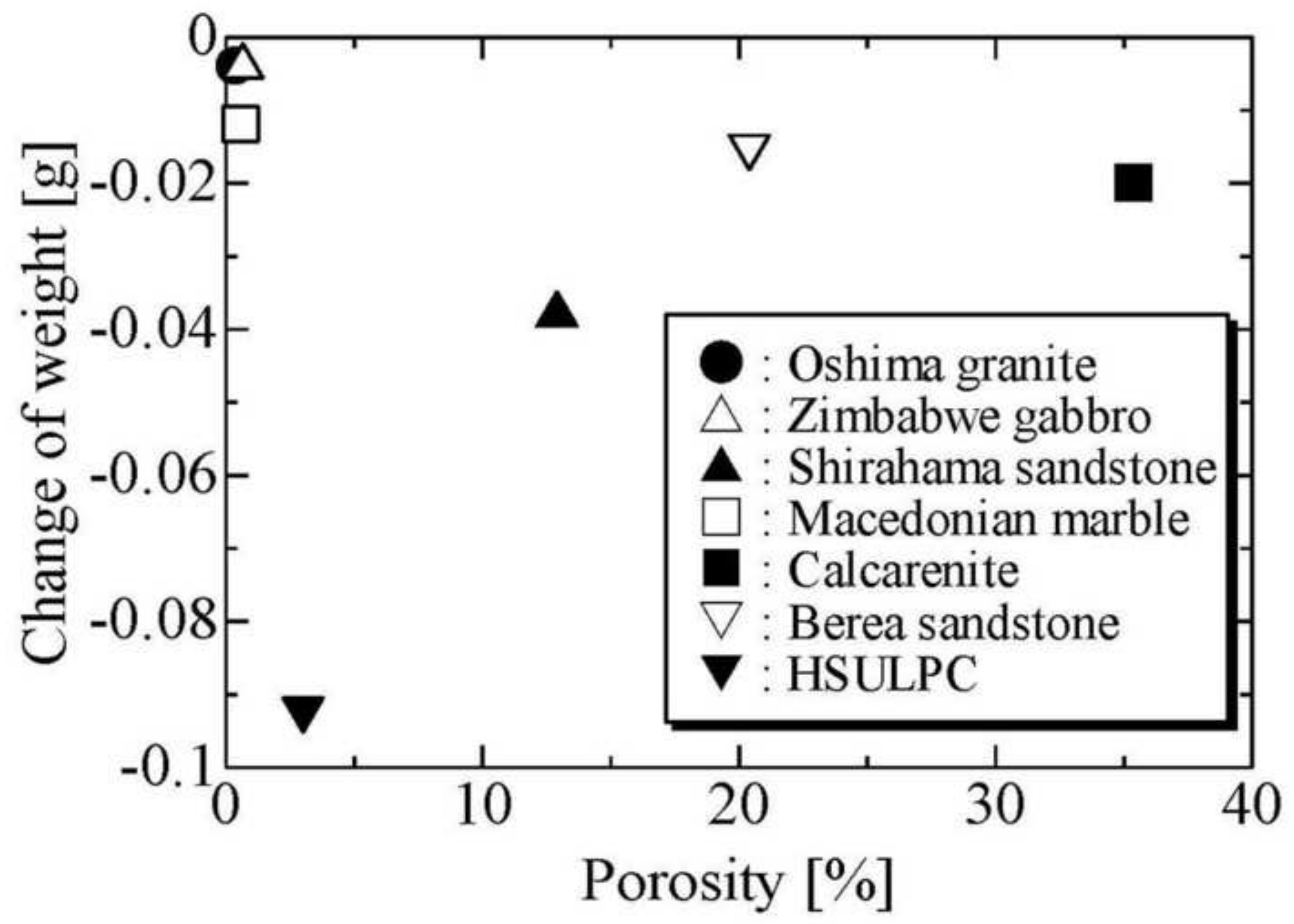

